\title{
Selective Separation of Phenanthrene from Aromatic Isomer Mixtures by a Water-Soluble Azobenzene-Based Macrocycle
}

Yuezhou Liü, Hongliang Wang\#, Liqing Shangguan, Peiren Liu, Bingbing Shi*, Xin Hong* and Feihe Huang*

\section{Y. Liu, L. Shangguan, P. Liu and Prof. Dr. F. Huang}

State Key Laboratory of Chemical Engineering, Center for Chemistry of High-Performance \& Novel Materials, Department of Chemistry, Zhejiang University, Hangzhou 310027, P. R. China; E-mail: fhuang@zju.edu.cn

H. Wang and Prof. Dr. X. Hong

Department of Chemistry, Zhejiang University, Hangzhou 310058, China; E-mail:

hxchem@zju.edu.cn

Prof. Dr. B. Shi

College of Chemistry and Chemical Engineering, Northwest Normal University, Lanzhou, 730070, China; E-Mail: bingbingshi@nwnu.edu.cn

Prof. Dr. F. Huang

Green Catalysis Center and College of Chemistry, Zhengzhou University, Zhengzhou 450001, P. R. China.

Prof. Dr. X. Hong

State Key Laboratory of Clean Energy Utilization, Zhejiang University, Zheda Road 38, Hangzhou 310027, China. 


\section{Table of Content ( 70 pages)}

1. Materials and instrumentations $\quad$ S3

2. Synthesis and characterization of compounds $\quad$ S4

3. Crystallographic data for E,E-2 S11

4. Concentration-variant ${ }^{1} H$ NMR spectra of E,E-1 S13

$\begin{array}{ll}\text { 5. Photoisomerization of } 1 & \text { S14 }\end{array}$

6. The host-guest complexation between 1 and ANT or PHE S16

7. Selective separation of PHE from aromatic isomer mixtures and $\begin{array}{ll}\text { cycling experiments } & \text { S21 }\end{array}$

8. The DFT-optimized structures of E,E-1・PHE and E,E-1•ANT S24

$\begin{array}{ll}\text { 9. References } & \mathrm{S} 24\end{array}$ 


\section{Materials and instrumentations}

All reagents were commercially available and used as supplied without further purification. Compound 3 was prepared according to the previous work. ${ }^{[\mathrm{S} 1]}{ }^{1} \mathrm{H}$ NMR, ${ }^{13} \mathrm{C}$ NMR, 2D NOESY and COSY were recorded with an Agilent $600 \mathrm{MHz}$ DirectDrive2 instrument with use of the deuterated solvent as the lock and the residual solvent or TMS as the internal reference. High-resolution mass spectra (HRMS) were performed with a Bruker 7-Tesla FT-ICR mass spectrometer equipped with an electrospray source (Billerica, MA, USA). Quadrupole time of flight mass spectrometry (Q-TOF MS) was performed with an Agilent 6200 quadrupole mass spectrometer. UV-vis spectra were taken on a PerkinElmer Lambda 35 UV-vis spectrophotometer. The MALDI-TOF data were collected on an Ultraflextrememass spectrometer of Bruker. Single crystal X-ray data were obtained on an Oxford Diffraction Xcalibur Atlas Gemini captra instrument.

All DFT calculations were performed using Gaussian 09 program. ${ }^{[\mathrm{S} 2]}$ Geometry optimizations were carried out using B3LYP ${ }^{[\mathrm{S} 3-\mathrm{S} 5]}$-D3 (Becke-Johnson damping function $)^{[\mathrm{S} 6, \mathrm{~S} 7]}$ functional, with def2-SVP basis $\operatorname{set}^{[\mathrm{S} 8, \mathrm{~S} 9]}$ for all elements. The vibrational frequency was calculated at the same level of theory to identify each optimized stationary point as an energy minimum or a transition state, and to evaluate the zero-point vibrational energy and thermal corrections at $298 \mathrm{~K}$. Based on the gas-phase optimized structures, the single-point energies and solvent effects of water were calculated using B3LYP-D3 functional and def2-TZVP basis set ${ }^{[\mathrm{S} 8, \mathrm{~S} 9]}$ for all elements, using the SMD solvation model. ${ }^{[\mathrm{S} 10]}$ The 3D diagrams of computed species were generated by CYLView. ${ }^{[\mathrm{S} 11]}$ 


\section{Synthesis and characterization of compounds}
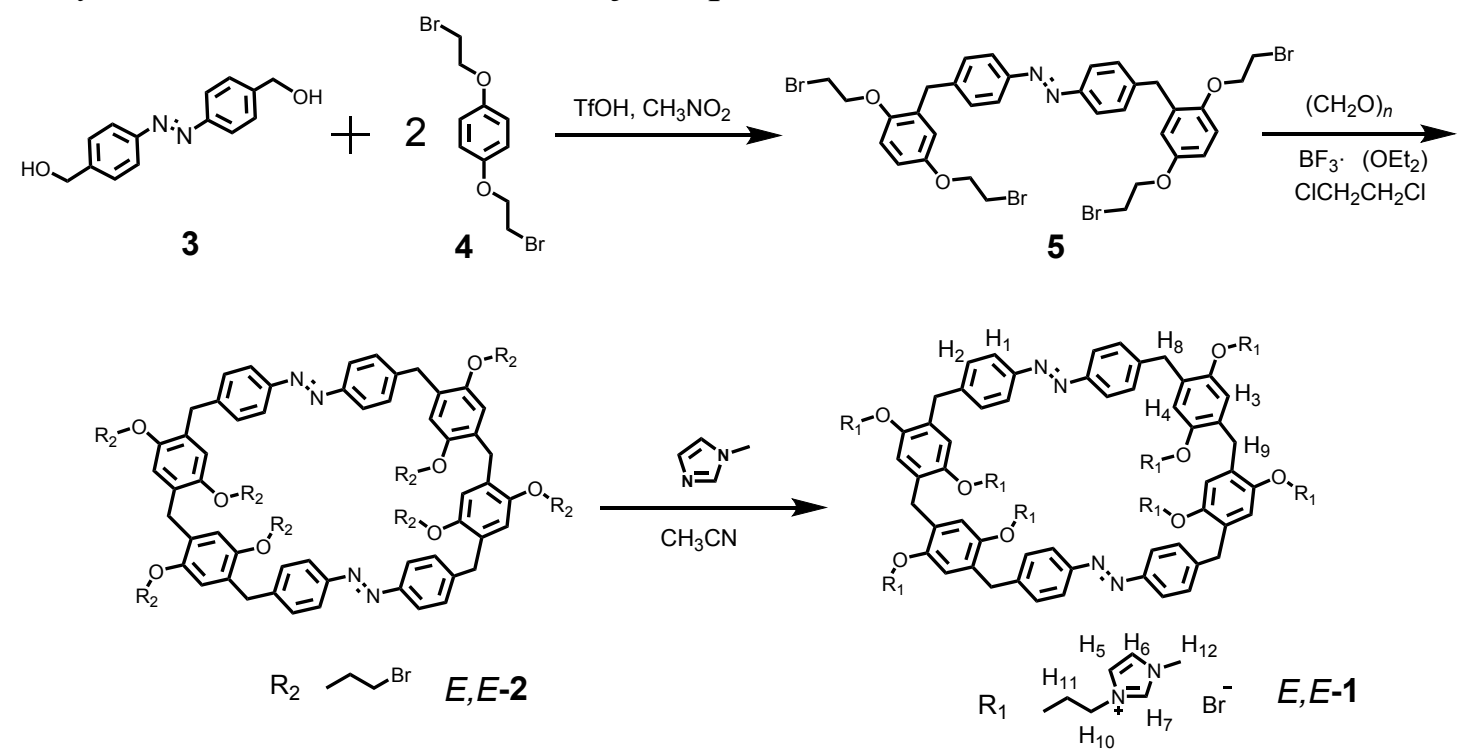

Scheme S1. Synthetic route to $E, E-1$.

Synthesis of compound 5 . Compound 3 was synthesized according to the previous work. ${ }^{[\mathrm{S} 1]}$ Compound 3 (1.00 g, $\left.4.95 \mathrm{mmol}\right)$, compound 4 (6.43 g, $\left.20.0 \mathrm{mmol}\right), 1.0 \mathrm{~mL}$ of trifluoromethanesulfonic acid and $120 \mathrm{~mL}$ of $\mathrm{CH}_{3} \mathrm{NO}_{2}$ were added to a $250 \mathrm{~mL}$ round-bottom flask and the reaction mixture was stirred under $\mathrm{N}_{2}$ atmosphere at 110 ${ }^{\circ} \mathrm{C}$ for $24 \mathrm{~h}$. Then the solvent was concentrated by rotary evaporation and the resulting residue was purified by column chromatography on silica gel using a mixture of petroleum ether and dichloromethane $(v: v=2: 1)$ as the eluent to give $1.25 \mathrm{~g}(30 \%$ yield) of 5 as an orange solid. Mp: $129.5-130.4{ }^{\circ} \mathrm{C}$. The ${ }^{1} \mathrm{H}$ NMR spectrum of compound 5 is shown in Figure $\mathrm{S} 1 .{ }^{1} \mathrm{H}$ NMR $\left(600 \mathrm{MHz}, \mathrm{CDCl}_{3}, 298 \mathrm{~K}\right) \delta(\mathrm{ppm})$ : 7.80-7.82 (d, $J=12 \mathrm{~Hz}, 4 \mathrm{H}), 7.35-7.37$ (d, $J=12 \mathrm{~Hz}, 4 \mathrm{H}), 6.72-6.78$ (m, $6 \mathrm{H})$, 4.19-4.23 (m, $8 \mathrm{H}), 4.04(\mathrm{~s}, 4 \mathrm{H}), 3.56-3.61(\mathrm{~m}, 8 \mathrm{H})$. The ${ }^{13} \mathrm{C}$ NMR spectrum of 5 is shown in Figure S2. ${ }^{13} \mathrm{C}$ NMR (150 MHz, $\left.\mathrm{CDCl}_{3}, 298 \mathrm{~K}\right) \delta(\mathrm{ppm}): 152.6,151.2$, $150.7,143.9,131.2,129.6,122.8,118.1,113.0,68.7,36.2,29.5$. The Q-TOF mass spectrum is shown in Figure $\mathrm{S} 3$ : calculated for $\left[\mathrm{M}+\mathrm{H}^{+}\right] \mathrm{C}_{34} \mathrm{H}_{35} \mathrm{Br}_{4} \mathrm{~N}_{2} \mathrm{O}_{4}{ }^{+}$850.9325, found 850.9326 , error $0.1 \mathrm{ppm}$. 

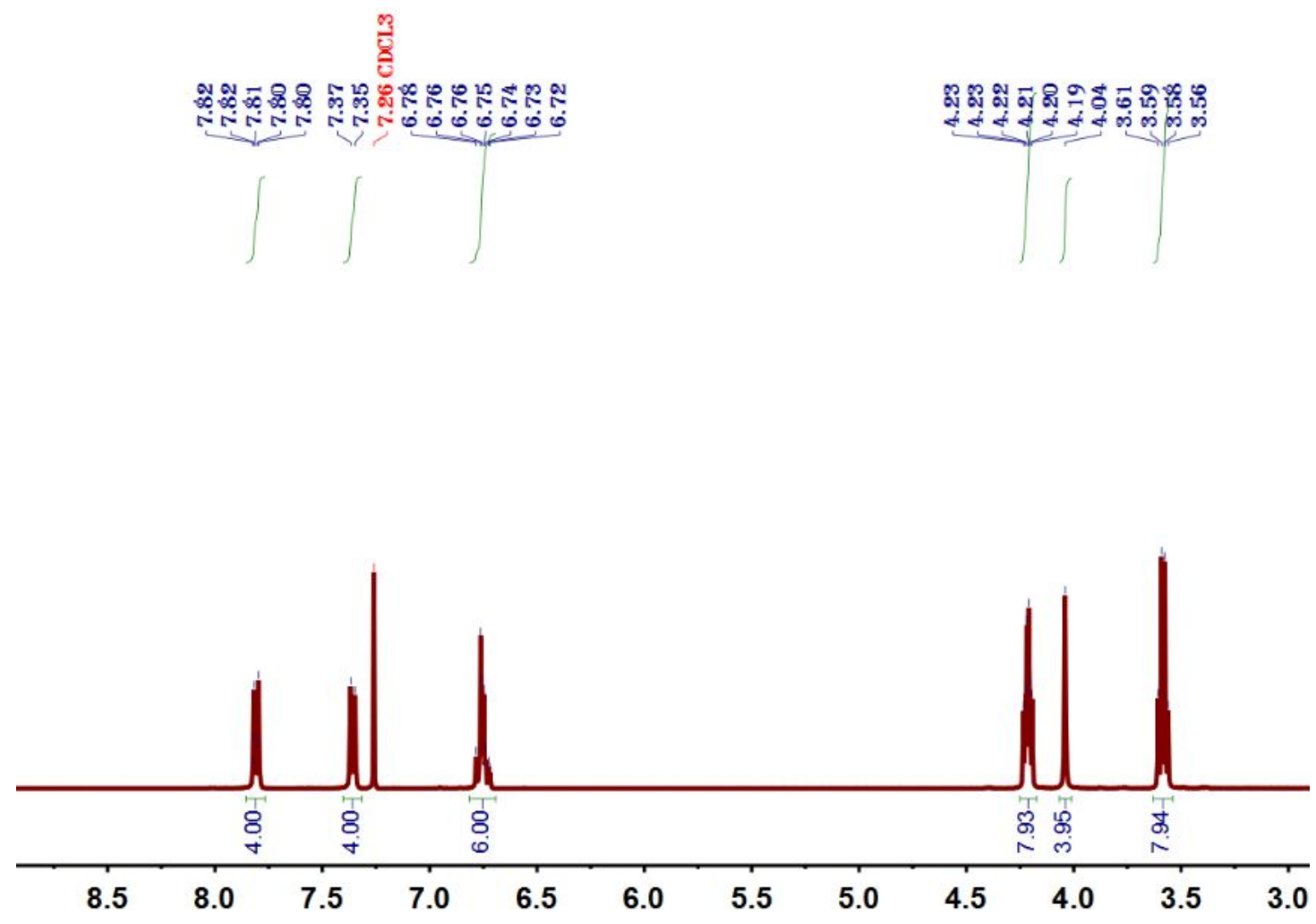

Figure S1 ${ }^{1} \mathrm{H}$ NMR spectrum $\left(600 \mathrm{MHz}, \mathrm{CDCl}_{3}, 298 \mathrm{~K}\right)$ of 5.

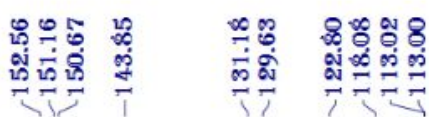
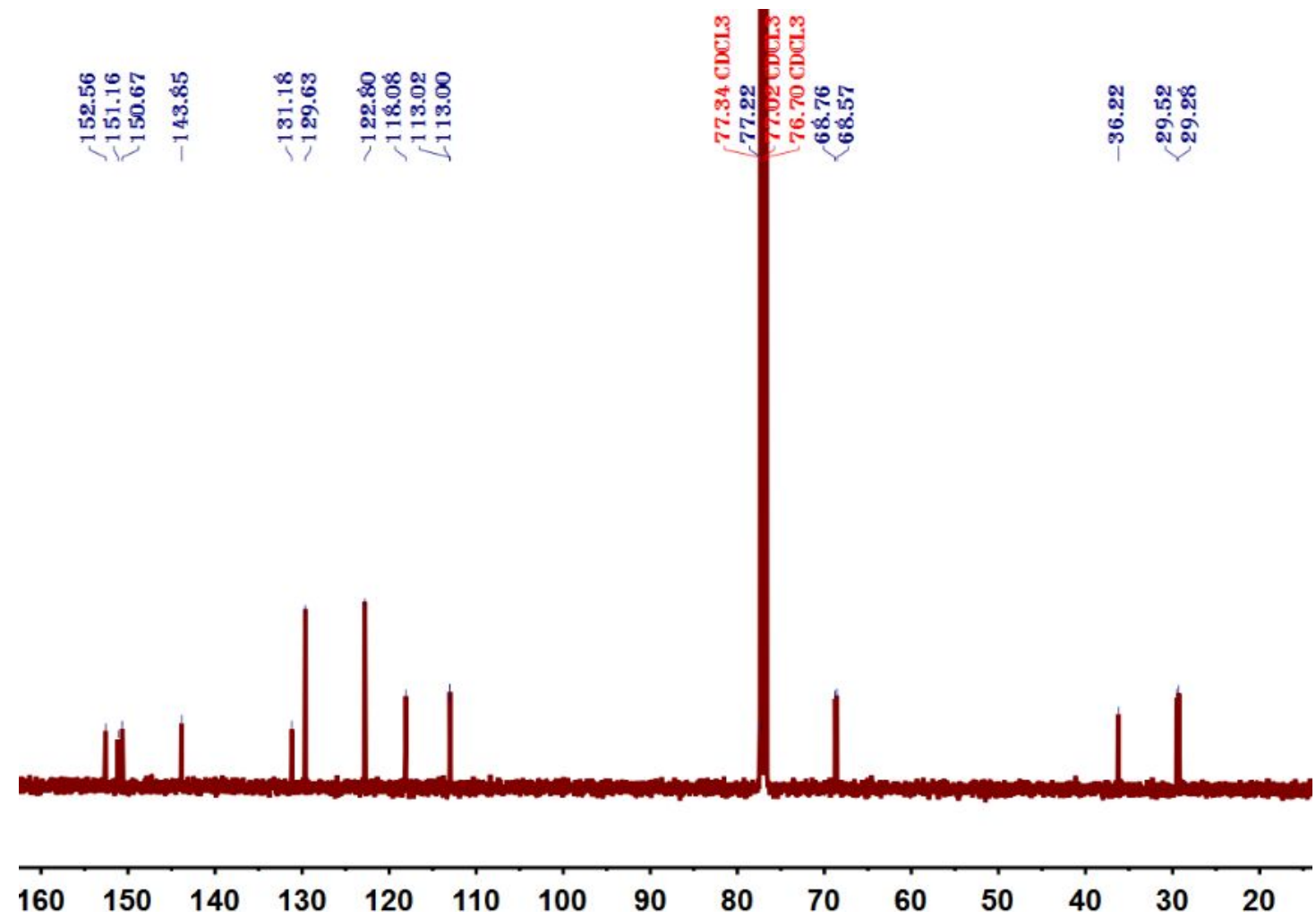

Figure S2 ${ }^{13} \mathrm{C}$ NMR spectrum $\left(150 \mathrm{MHz}, \mathrm{CDCl}_{3}, 298 \mathrm{~K}\right)$ of 5 . 


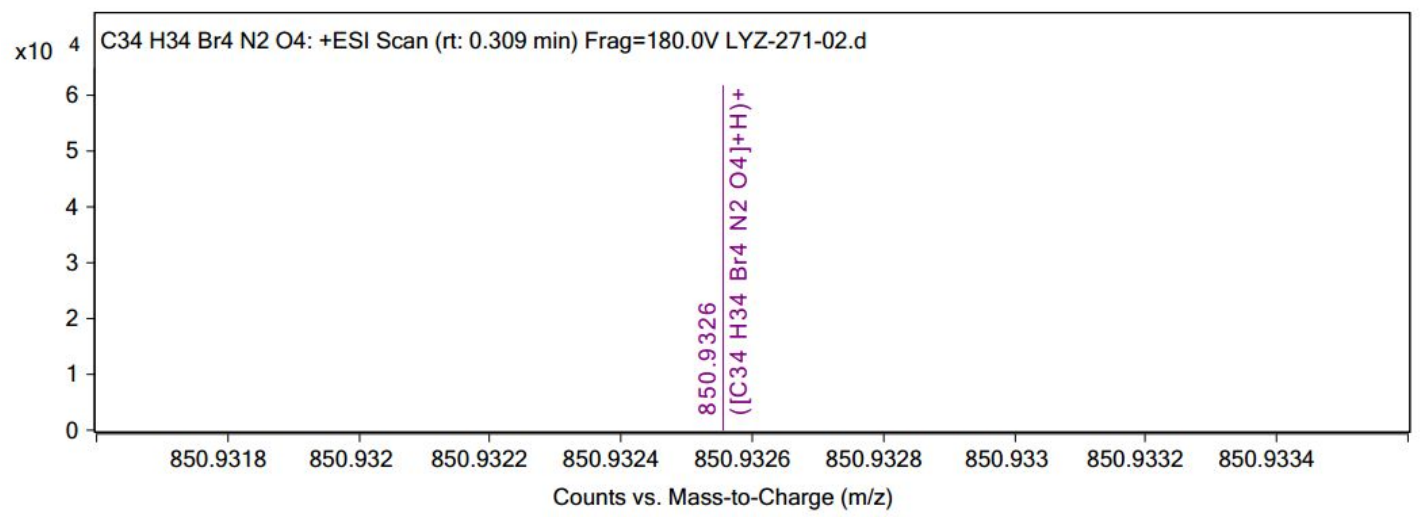

Figure S3 Q-TOF mass spectrum of 5.

Synthesis of $\boldsymbol{E}, \boldsymbol{E}-2$. To a $100 \mathrm{~mL}$ round bottom flask, compound 5 (1020 mg, 1.20 mmol), paraformaldehyde (108 mg, $3.6 \mathrm{mmol}$ ) and $60 \mathrm{~mL}$ of 1,2-dichloroethane were added. $\mathrm{BF}_{3} \cdot \mathrm{O}\left(\mathrm{C}_{2} \mathrm{H}_{5}\right)_{2}(1.0 \mathrm{~mL}, 7.8 \mathrm{mmol})$ was added to the solution and the mixture was stirred at room temperature for $1.5 \mathrm{~h}$. The reaction was quenched with water. The organic layer was washed with water and saturated $\mathrm{NaCl}$ solution, and dried with anhydrous $\mathrm{Na}_{2} \mathrm{SO}_{4}$. After concentrating the dried organic layer to a minimum volume by rotary evaporation, the resulting residue was purified by column chromatography on silica gel using a mixture of petroleum ether and ethyl acetate $(v: v=10: 1)$ as the eluent to give $320 \mathrm{mg}$ (31\% yield) of E,E-2 as an orange solid. Mp: $194.2-195.3{ }^{\circ} \mathrm{C}$. The ${ }^{1} \mathrm{H}$ NMR spectrum of $E, E-2$ is shown in Figure S4. ${ }^{1} \mathrm{H}$ NMR $\left(600 \mathrm{MHz}, \mathrm{CDCl}_{3}\right.$, $298 \mathrm{~K}) \delta(\mathrm{ppm}): 7.71-7.72(\mathrm{~d}, J=6 \mathrm{~Hz}, 8 \mathrm{H}), 7.28-7.30(\mathrm{~d}, J=12 \mathrm{~Hz}, 8 \mathrm{H}), 6.90(\mathrm{~s}, 4$ H), 6.67 (s, $4 \mathrm{H}), 4.22-4.24$ (t, $J=6 \mathrm{~Hz}, 8 \mathrm{H}), 4.16-4.18$ (t, $J=6 \mathrm{~Hz}, 8 \mathrm{H}), 3.97-3.99$ (d, $J=12 \mathrm{~Hz}, 12 \mathrm{H}), 3.62-3.64$ (t, $J=6 \mathrm{~Hz}, 8 \mathrm{H}), 3.49-3.51$ (t, $J=6 \mathrm{~Hz}, 8 \mathrm{H})$. The ${ }^{13} \mathrm{C}$ NMR spectrum of $E, E-2$ is shown in Figure S5. ${ }^{13} \mathrm{C}$ NMR $\left(150 \mathrm{MHz}, \mathrm{CDCl}_{3}, 298\right.$ K) $\delta(\mathrm{ppm}): 153.7,153.0,152.9,146.9,131.9,130.8,125.3,118.7,118.0,71.5,71.7$, $39.0,33.0,32.8,32.3$. The MALDI-TOF-MS spectrum of $E, E-2$ is shown in Figure S6. $m / z$ of $\left[\mathrm{M}+\mathrm{H}^{+}\right] \mathrm{C}_{70} \mathrm{H}_{69} \mathrm{Br}_{8} \mathrm{~N}_{4} \mathrm{O}_{8}{ }^{+} 1732.2443$. 


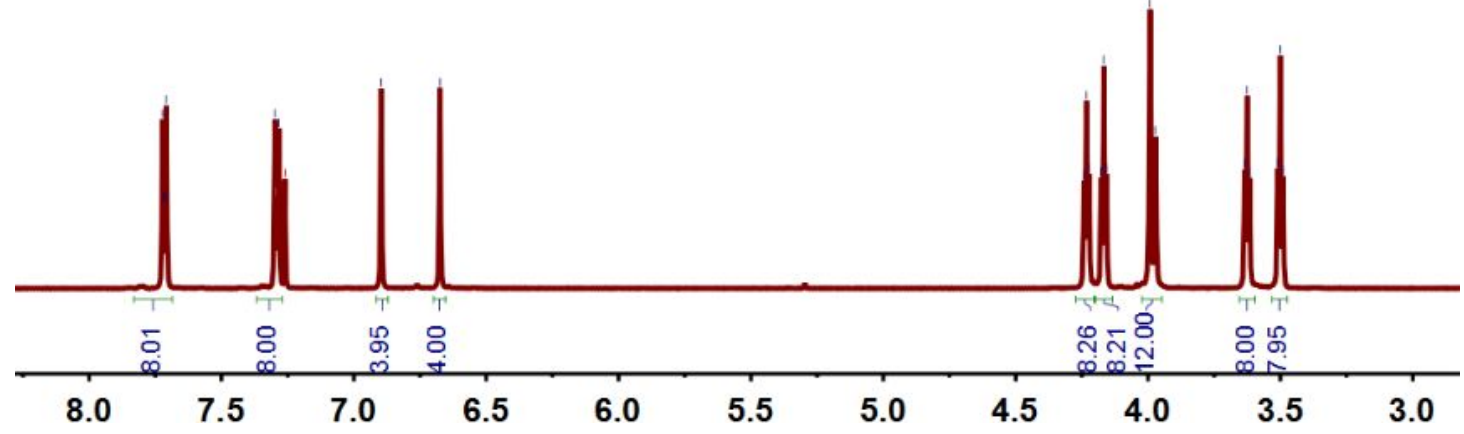

Figure $\mathbf{S 4}{ }^{1} \mathrm{H}$ NMR spectrum $\left(600 \mathrm{MHz}, \mathrm{CDCl}_{3}, 298 \mathrm{~K}\right)$ of $E, E-2$.

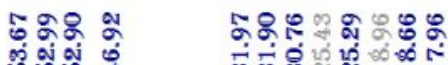

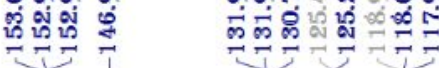

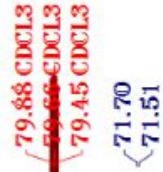

Кำระ

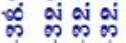

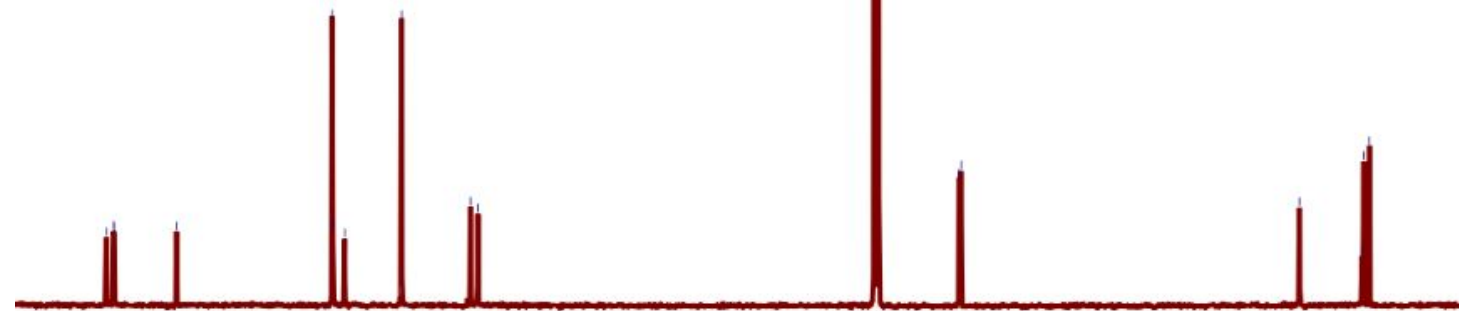

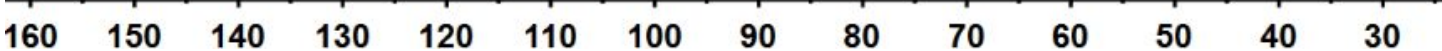

Figure S5 ${ }^{13} \mathrm{C}$ NMR spectrum $\left(150 \mathrm{MHz}, \mathrm{CDCl}_{3}, 298 \mathrm{~K}\right)$ of $E, E-2$. 


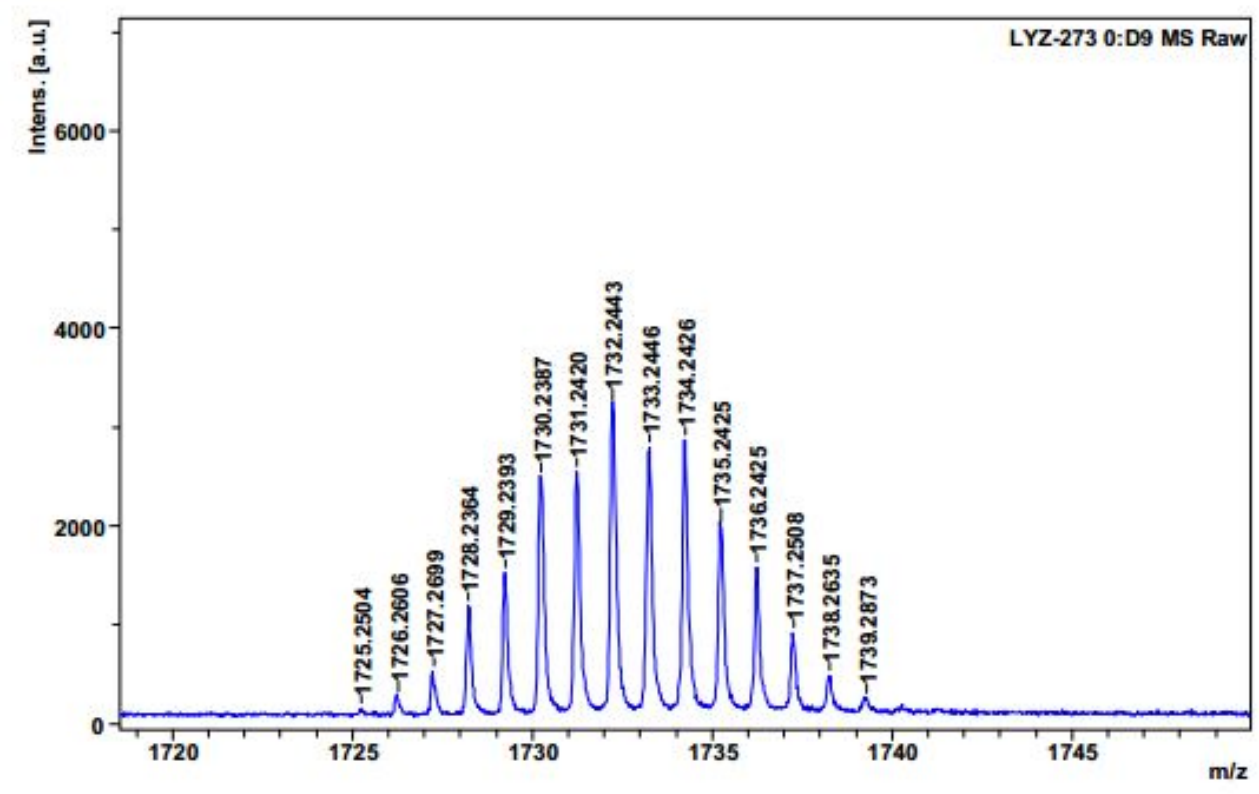

Figure S6. The MALDI-TOF-MS spectrum of E,E-2.

Synthesis of $\boldsymbol{E}, \boldsymbol{E}-1$. A mixture of $E, E-2(1.73 \mathrm{~g}, 1.00 \mathrm{mmol})$ and $N$-methylimidazole (1.64 g, $20.0 \mathrm{mmol})$ in $\mathrm{CH}_{3} \mathrm{CN}(25 \mathrm{~mL})$ was stirred in a $50 \mathrm{~mL}$ round-bottom flask at $85{ }^{\circ} \mathrm{C}$ for 24 hours. After cooling, the solvent was removed and the residue was dissolved in a small quantity of $\mathrm{CH}_{3} \mathrm{CH}_{2} \mathrm{OH}$, and the solution was precipitated in diethyl ether to give an orange solid (1.95 g, $82 \%)$. The ${ }^{1} \mathrm{H}$ NMR spectra of $E, E-1$ are shown in Figure S7 and Figure S8. ${ }^{1} \mathrm{H}$ NMR $\left(600 \mathrm{MHz}, \mathrm{D}_{2} \mathrm{O}, 298 \mathrm{~K}\right) \delta$ (ppm): 7.51-7.53 (d, J=12 Hz, 8 H), 7.27 (s, 4 H), 7.20 (s, 4 H), 7.10 (s, 4 H), 7.02-7.05 (t, $J=9 \mathrm{~Hz}, 12 \mathrm{H}), 6.84$ (s, $4 \mathrm{H}), 6.51$ (s, $4 \mathrm{H}), 4.41-4.42$ (t, $J=3 \mathrm{~Hz}, 8 \mathrm{H}), 4.31-4.33$ (t, $J=6 \mathrm{~Hz}, 8 \mathrm{H}), 4.26-4.28(\mathrm{t}, J=6 \mathrm{~Hz}, 8 \mathrm{H}), 4.05-4.07(\mathrm{t}, J=6 \mathrm{~Hz}, 8 \mathrm{H}), 3.83(\mathrm{~s}, 8 \mathrm{H})$, 3.70 (s, $4 \mathrm{H}), 3.51$ (s, $12 \mathrm{H}), 3.48$ (s, $12 \mathrm{H}) .{ }^{1} \mathrm{H}$ NMR (600 MHz, DMSO-d $\left.d_{6}, 298 \mathrm{~K}\right) \delta$ (ppm): 9.43 (s, 4 H), 9.31 (s, 4 H), 7.64-7.91 (m, 24 H), 7.09-7.18 (m, 12 H), 6.67 (s, $4 \mathrm{H}), 4.57-4.69$ (m, $16 \mathrm{H}), 4.20-4.39(\mathrm{~m}, 16 \mathrm{H}), 3.63-3.88(\mathrm{~m}, 36 \mathrm{H})$. The ${ }^{13} \mathrm{C} \mathrm{NMR}$ spectrum of $E, E-1$ is shown in Figure S9. ${ }^{13} \mathrm{C} \mathrm{NMR}\left(150 \mathrm{MHz}, \mathrm{DMSO}-d_{6}, 298 \mathrm{~K}\right) \delta$ (ppm): 150.1, 149.7, 149.5, 144.4, 137.0, 136.8, 129.1, 128.0, 123.4, 122.7, 122.3, $115.8,67.0,66.2,48.7,35.8$. HRMS of $E, E-1$ is shown in Figure S10: $\mathrm{m} / \mathrm{z}$ of $\left[\mathrm{M}-4 \mathrm{Br}^{-}\right]^{4+} \mathrm{C}_{102} \mathrm{H}_{116} \mathrm{~N}_{20} \mathrm{O}_{8} \mathrm{Br}_{4}{ }^{4+}$ 517.9031; [M-5Br ${ }^{-5+} \mathrm{C}_{102} \mathrm{H}_{116} \mathrm{~N}_{20} \mathrm{O}_{8} \mathrm{Br}_{3}{ }^{5+}$ 398.1397. 


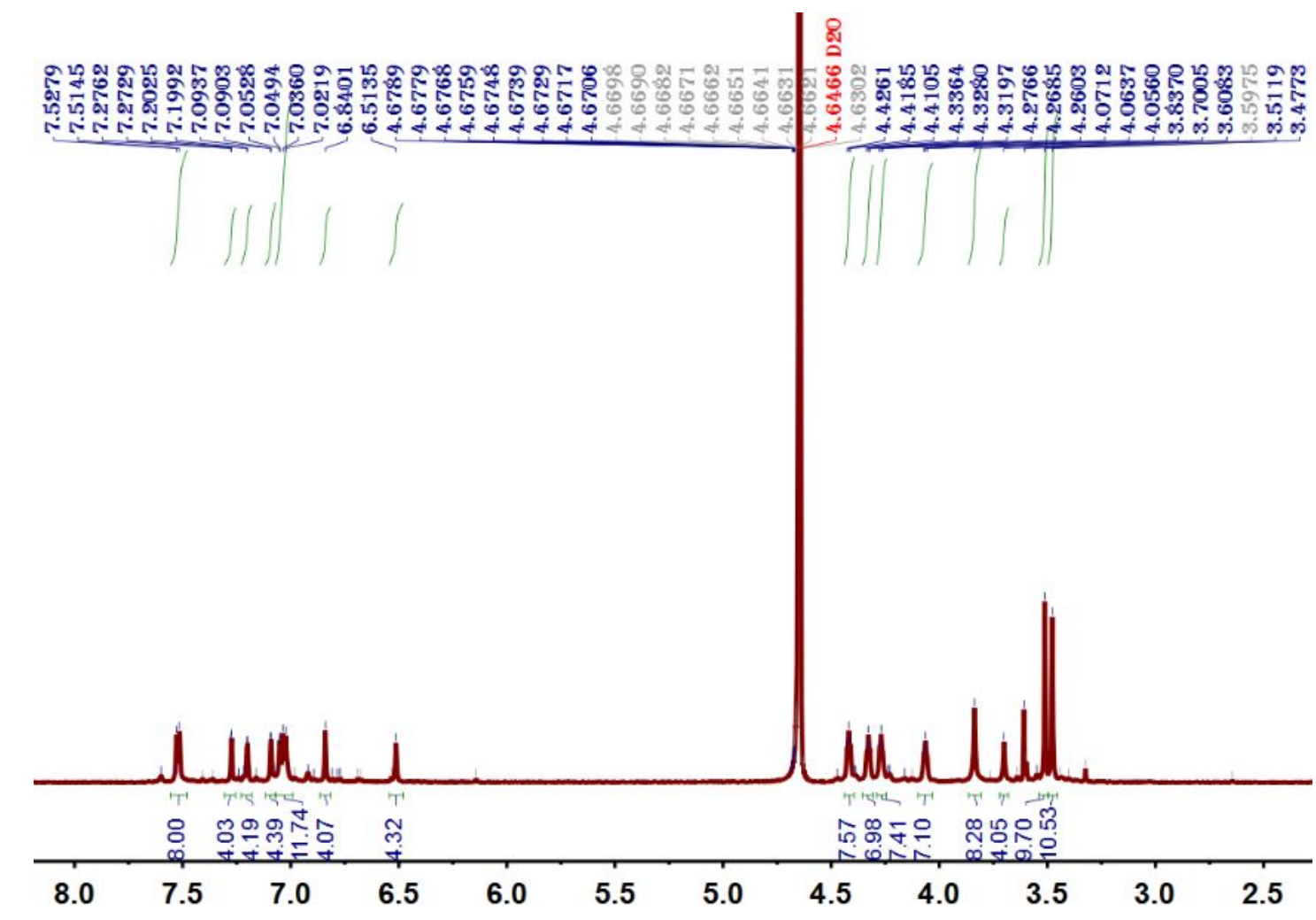

Figure S7 ${ }^{1} \mathrm{H}$ NMR spectrum $\left(600 \mathrm{MHz}, \mathrm{D}_{2} \mathrm{O}, 298 \mathrm{~K}\right)$ of $E, E-1$.

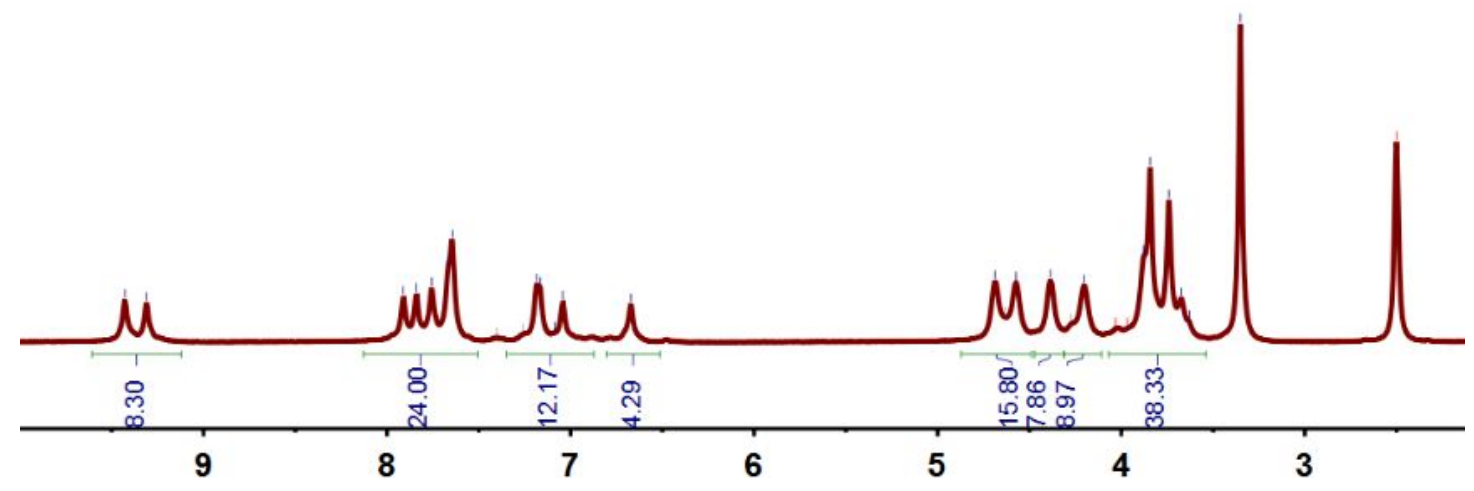

Figure S8 ${ }^{1} \mathrm{H}$ NMR spectrum $\left(600 \mathrm{MHz}, \mathrm{DMSO}-d_{6}, 298 \mathrm{~K}\right)$ of $E, E-1$. 


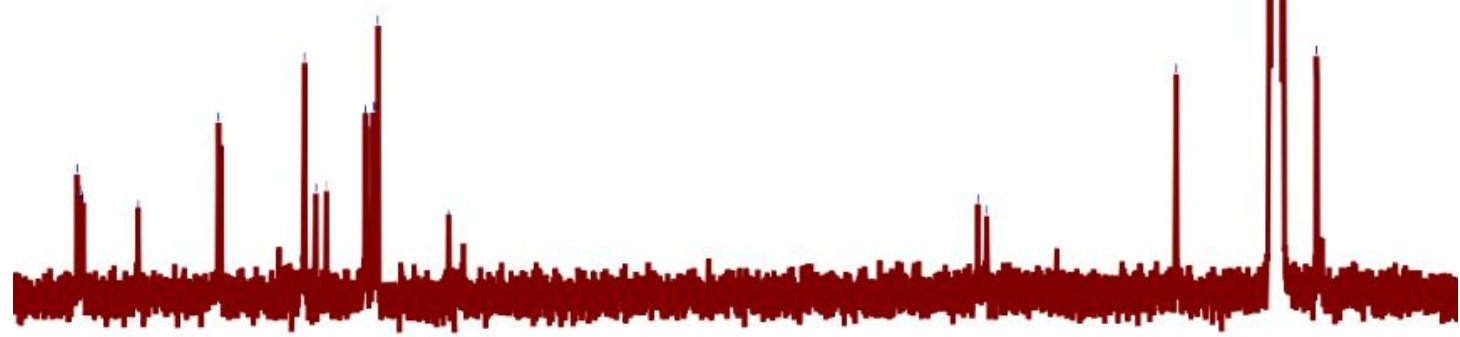

$\begin{array}{lllllllllllll}150 & 140 & 130 & 120 & 110 & 100 & 90 & 80 & 70 & 60 & 50 & 40 & 30\end{array}$

Figure S9 ${ }^{13} \mathrm{C}$ NMR spectrum $\left(150 \mathrm{MHz}, \mathrm{DMSO}-d_{6}, 298 \mathrm{~K}\right)$ of $E, E-1$.
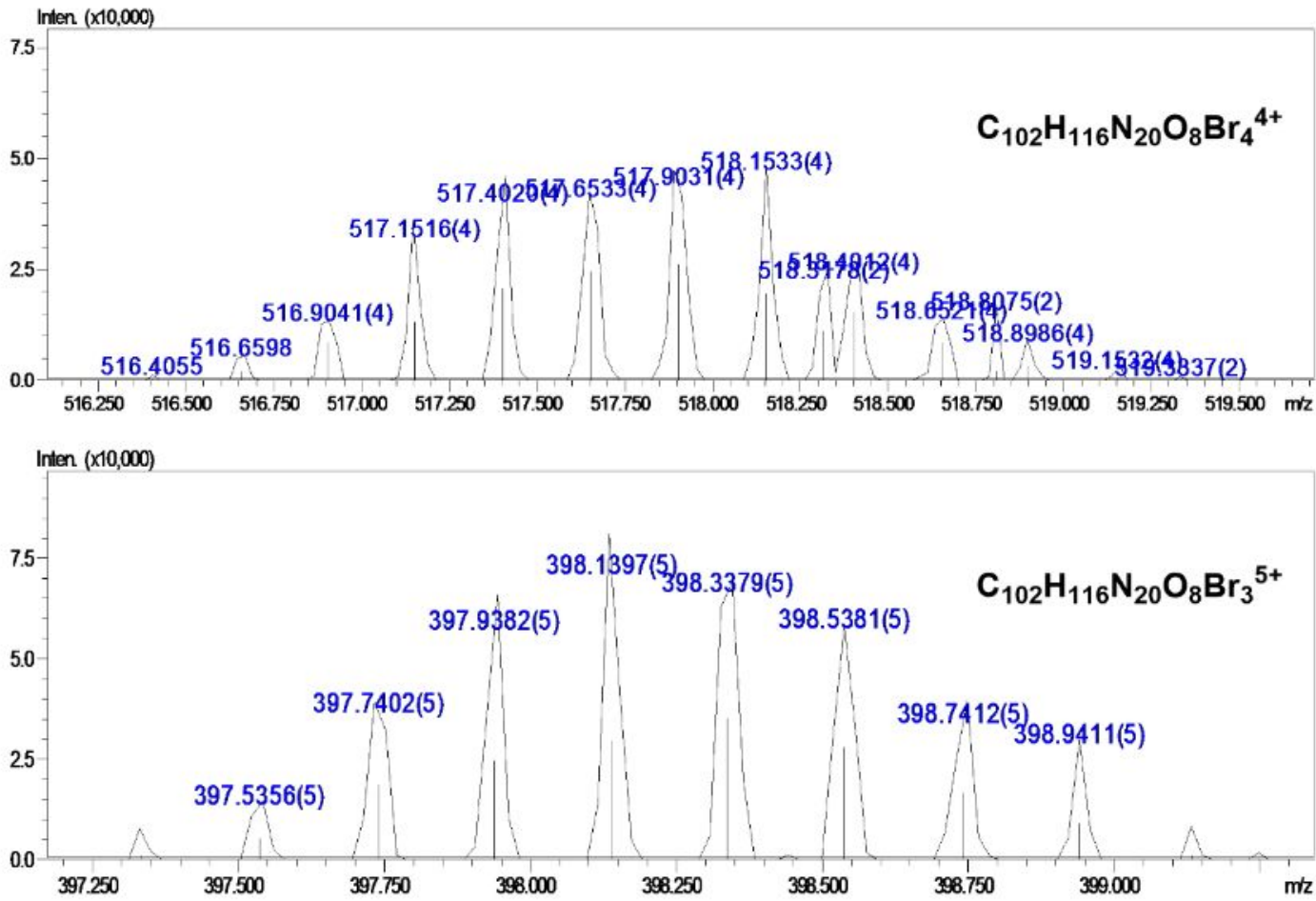

Figure S10 HRMS of $E, E-1$. 


\section{Crystallographic data for E,E-2}

Method to get single crystals of $E, E-2$ suitable for X-ray analysis: $E, E-2(10 \mathrm{mg})$ was dissolved in acetonitrile to make a hot saturated acetonitrile solution. The resultant solution was allowed to evaporate slowly to give orange $E, E-2$ crystals within 2 days.

Table S1. Experimental single crystal X-ray data for $E, E-2$.

\begin{tabular}{|c|c|}
\hline Formula & $E, E-\mathbf{2}$ \\
\hline Crystallization Solvent & acetonitrile \\
\hline Collection Temperature & 169.98 \\
\hline Formula & $\mathrm{C}_{76} \mathrm{H}_{77} \mathrm{Br}_{8} \mathrm{~N}_{7} \mathrm{O}_{8}$ \\
\hline$M r$ & 1855.72 \\
\hline Crystal Size $\left[\mathrm{mm}^{3}\right]$ & $0.06 \times 0.06 \times 0.05$ \\
\hline Crystal System & triclinic \\
\hline Space Group & P-1 \\
\hline$a[\AA]$ & $13.0738(12)$ \\
\hline$b[\AA]$ & $13.1691(10)$ \\
\hline$c[\AA]$ & $24.798(2)$ \\
\hline$\alpha\left[^{\circ}\right]$ & $77.425(5)$ \\
\hline$\beta\left[^{\circ}\right]$ & $79.198(6)$ \\
\hline$\gamma\left[^{\circ}\right]$ & $65.541(4)$ \\
\hline$V\left[\AA^{3}\right]$ & $3770.3(6)$ \\
\hline$Z$ & 2 \\
\hline Dcalcd $\left[\mathrm{g} \mathrm{cm}^{-3}\right]$ & 1.635 \\
\hline$\mu\left[\mathrm{mm}^{-1}\right]$ & 3.835 \\
\hline $\mathrm{F}(000)$ & 1852.0 \\
\hline $2 \theta$ range $\left[^{\circ}\right]$ & 6.496 to 110.314 \\
\hline Reflections collected & 41829 \\
\hline Independent reflections, Rint & $14195,0.0790$ \\
\hline
\end{tabular}


Data / restraints / parameters 14195/157/892

Final $R 1$ values $(I>2 \sigma(I)) \quad 0.1433$

Final $R 1$ values (all data) $\quad 0.2139$

Goodness-of-fit on $F^{2} \quad 0.901$

Largest difference peak and hole $\left[\mathrm{e} . \mathrm{A}^{-3}\right]$

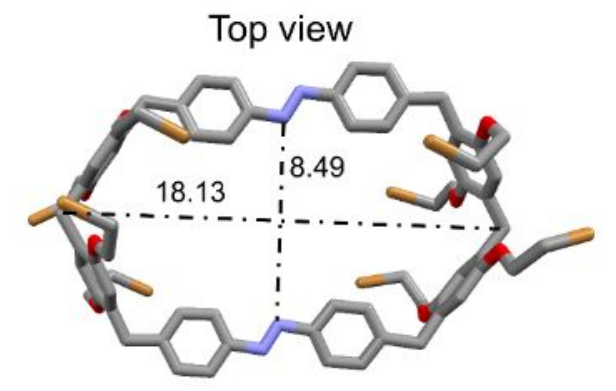

Side view

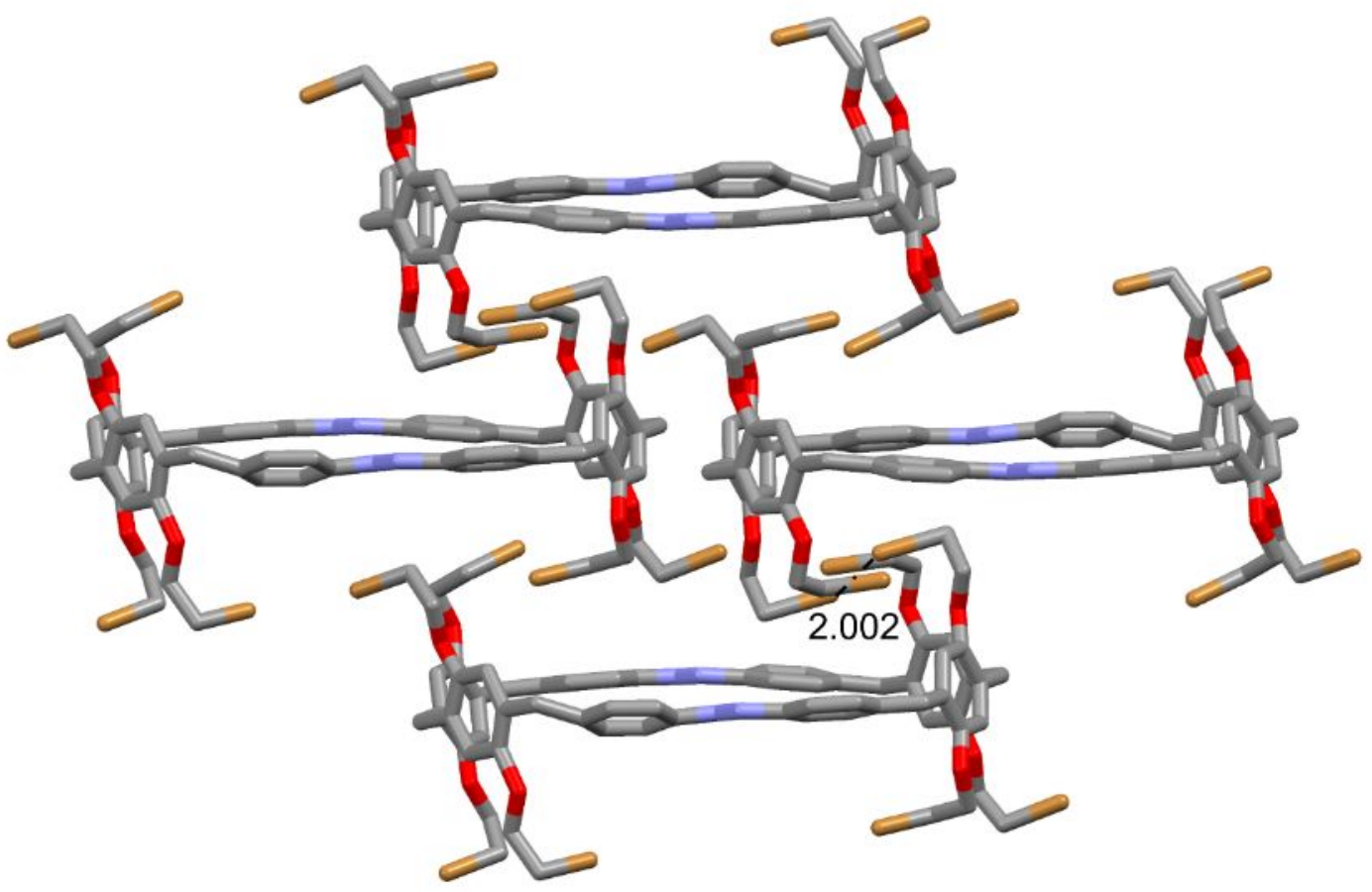

Figure S11 Single crystal structure of E,E-2. C gray, $\mathrm{O}$ red, $\mathrm{N}$ blue. A $\mathrm{Br} \cdots \mathrm{Br}$ interaction with a $\mathrm{Br} \cdots \mathrm{Br}$ distance of $2.002 \AA$. Hydrogen atoms are omitted for clarity. 


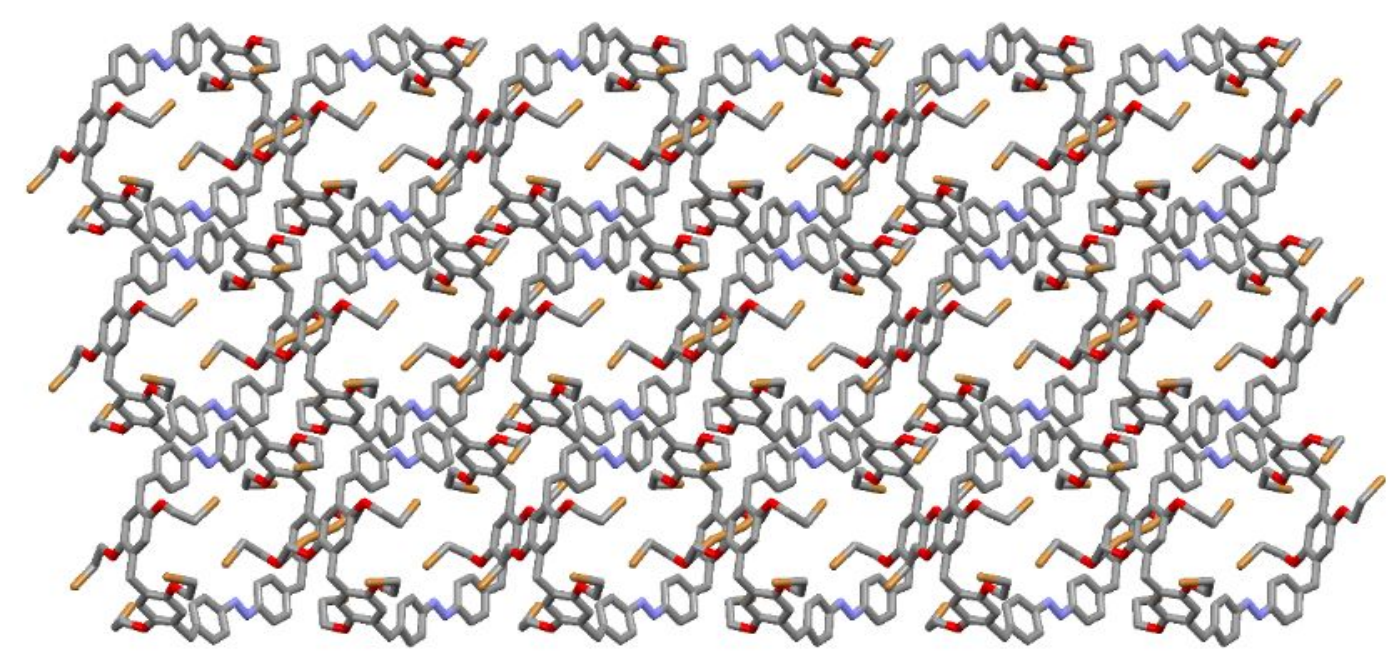

Figure S12 The packing structure of $E, E-2$ (view along $b$ axis). Hydrogen atoms are omitted for clarity.

\section{Concentration-variant ${ }^{1} H$ NMR spectra of E,E-1}

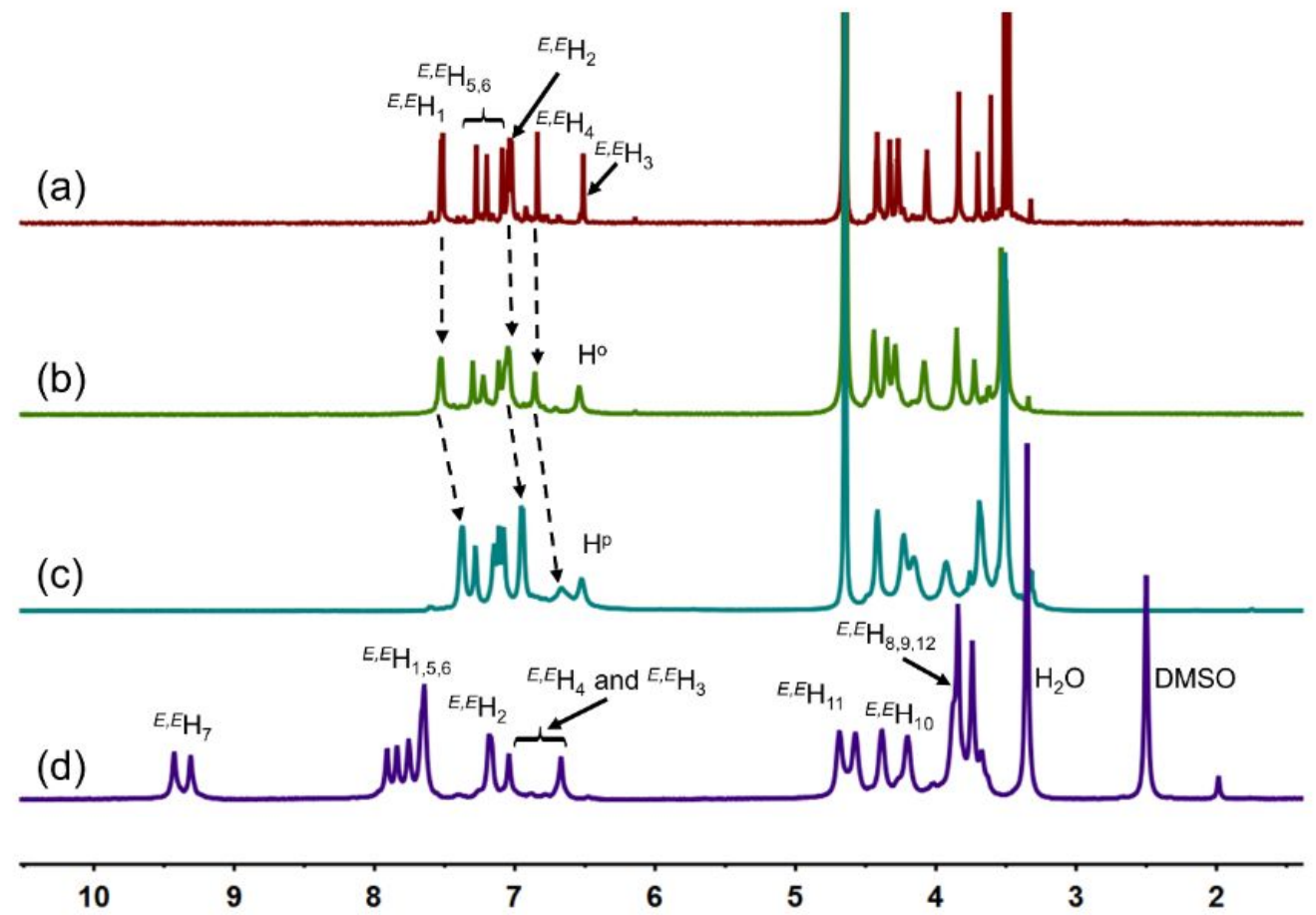

Figure $\mathbf{S 1 3}{ }^{1} \mathrm{H}$ NMR spectra $\left(600 \mathrm{MHz}, 298 \mathrm{~K}, \mathrm{D}_{2} \mathrm{O}\right)$ of $E, E-1$ at various concentrations: (a) $1.00 \mathrm{mM}$; (b) $3.00 \mathrm{mM}$; (c) $10.0 \mathrm{mM}$. (d) ${ }^{1} \mathrm{H}$ NMR spectrum (600 $\left.\mathrm{MHz}, 298 \mathrm{~K}, \mathrm{DMSO}-d_{6}\right)$ of $E, E-\mathbf{1}$. Peaks of supramolecular oligomer and polymer are designated by o and $p$, respectively. 


\section{Photoisomerization of 1}

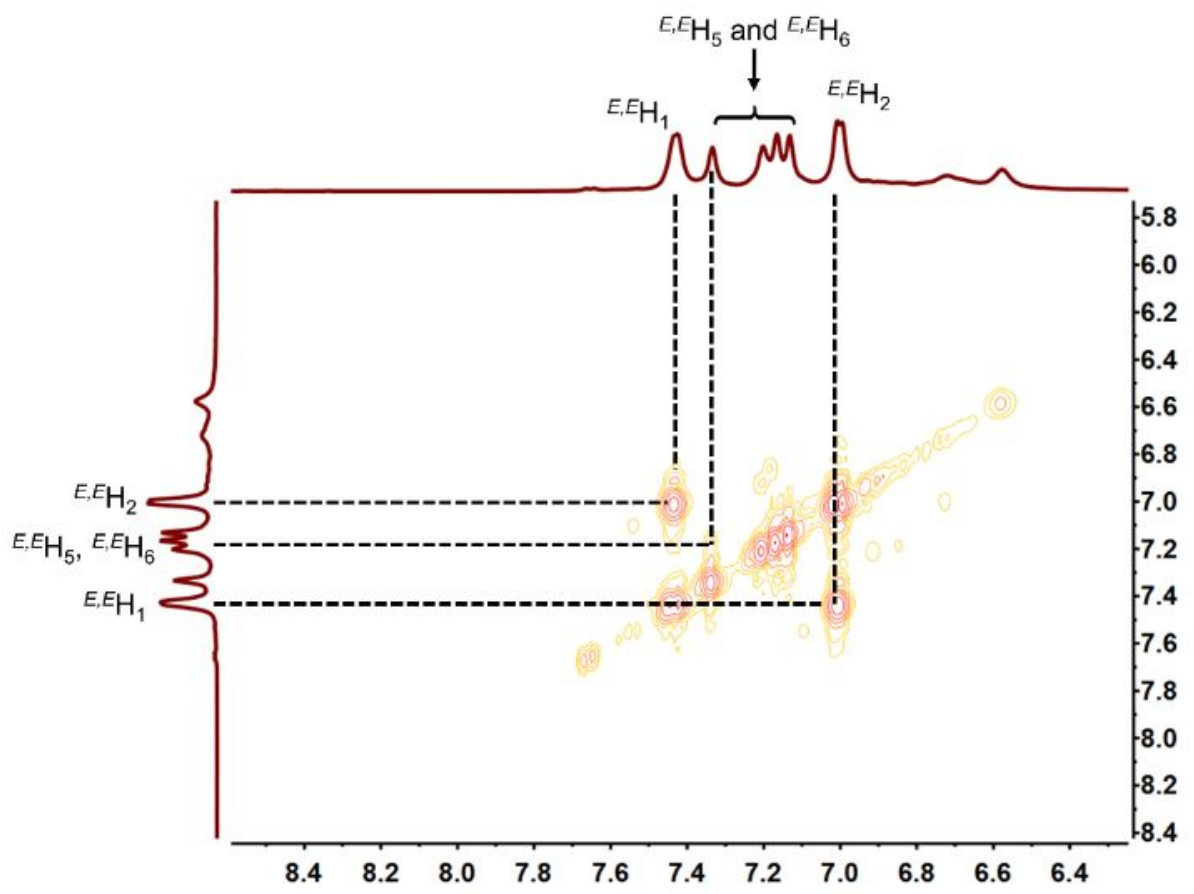

Figure $\mathrm{S14}$ COSY ${ }^{1} \mathrm{H}$ NMR spectrum $\left(\mathrm{D}_{2} \mathrm{O}, 600 \mathrm{MHz}\right.$, aromatic-aromatic resonance correlation) of $E, E-\mathbf{1}$.

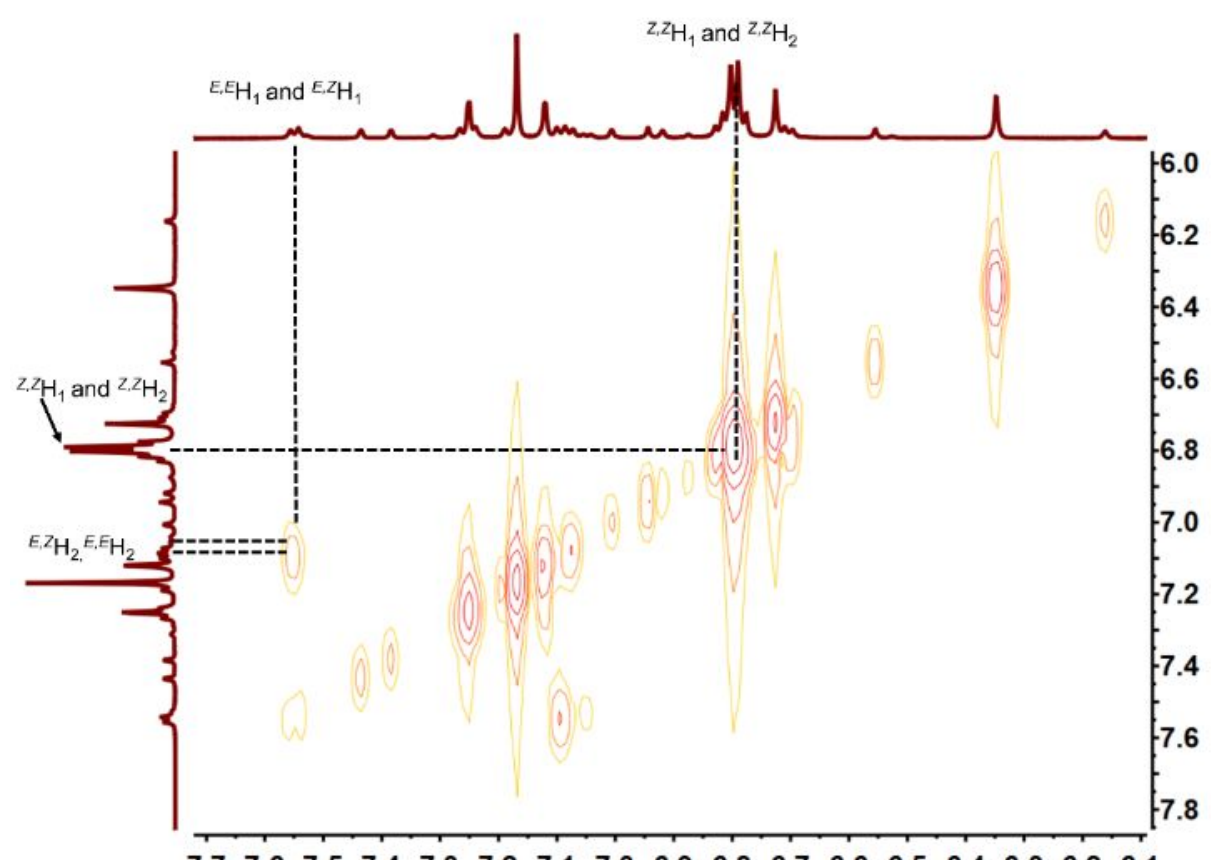

$\begin{array}{lllllllllllllllll}7.7 & 7.6 & 7.5 & 7.4 & 7.3 & 7.2 & 7.1 & 7.0 & 6.9 & 6.8 & 6.7 & 6.6 & 6.5 & 6.4 & 6.3 & 6.2 & 6.1\end{array}$

Figure S15 COSY ${ }^{1} \mathrm{H}$ NMR spectrum $\left(\mathrm{D}_{2} \mathrm{O}, 600 \mathrm{MHz}\right.$, aromatic-aromatic resonance correlation) of 1 upon partial Z-conversion via irradiation at $365 \mathrm{~nm}$. 
(a)

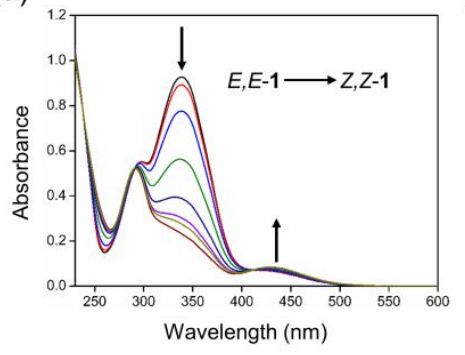

(b)

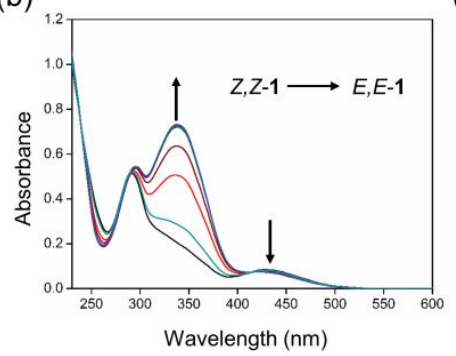

(c)

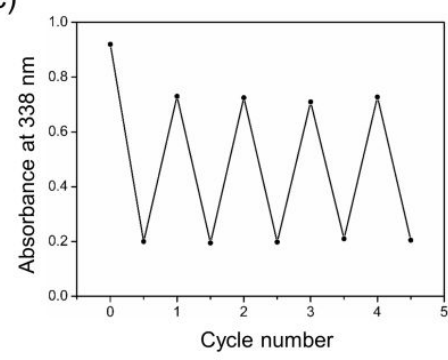

Figure S16 UV-vis absorption spectra $(20 \mu \mathrm{M}$ in water, $298 \mathrm{~K})$ of 1: upon increasing UV $365 \mathrm{~nm}$ irradiation for $1 \mathrm{~min}$ to achieve the $Z$-photostationary state and $420 \mathrm{~nm}$ for 1 min to achieve the $E$-photostationary state, and their absorbance intensity changes at $338 \mathrm{~nm}$ upon alternating UV light (365 nm) and visible light (420 nm) irradiations.

(a)

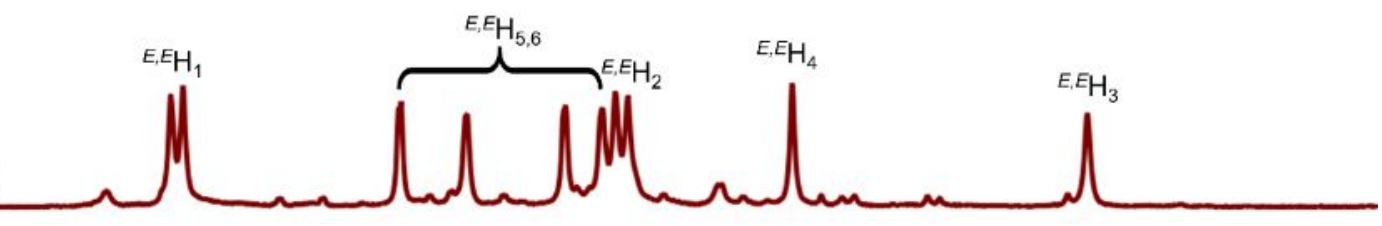

(b)
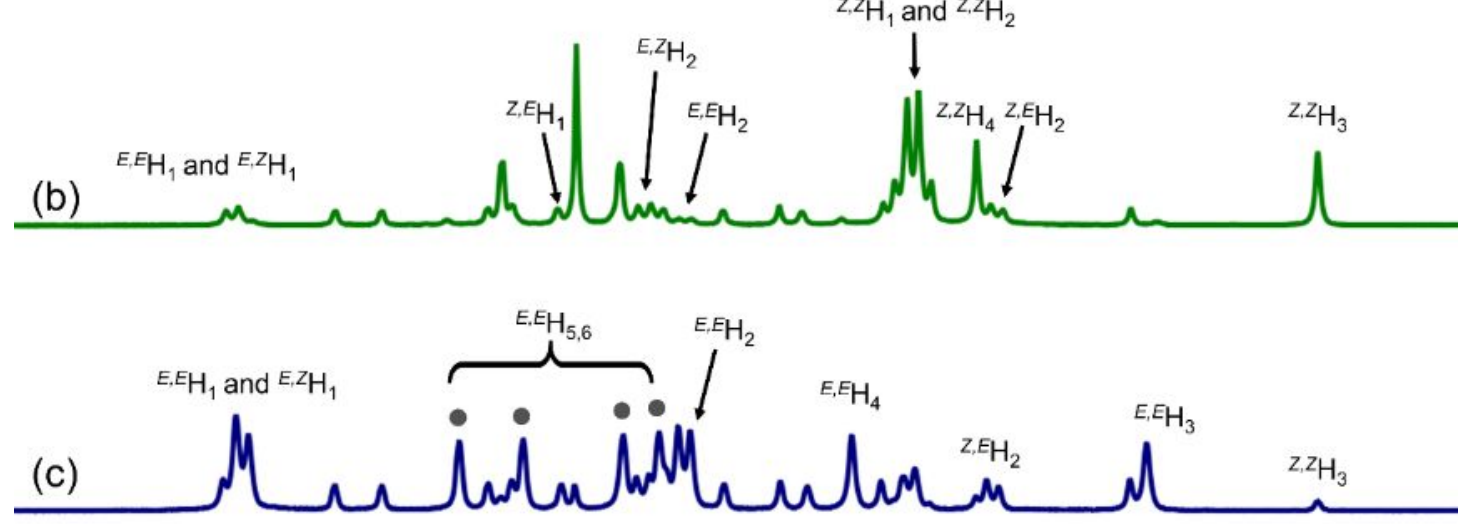

$\begin{array}{llllllllllllllll}7.7 & 7.6 & 7.5 & 7.4 & 7.3 & 7.2 & 7.1 & 7.0 & 6.9 & 6.8 & 6.7 & 6.6 & 6.5 & 6.4 & 6.3 & 6 .\end{array}$

Figure S17 Partial ${ }^{1} \mathrm{H}$ NMR spectra $\left(600 \mathrm{MHz}, \mathrm{D}_{2} \mathrm{O}, 298 \mathrm{~K}\right)$ of (a) $E, E-1$, (b) after photoirradiation (365 nm), and (c) after further photoirradiation (420 nm) of (b). 


\section{The host-guest complexation between 1 and ANT or PHE}

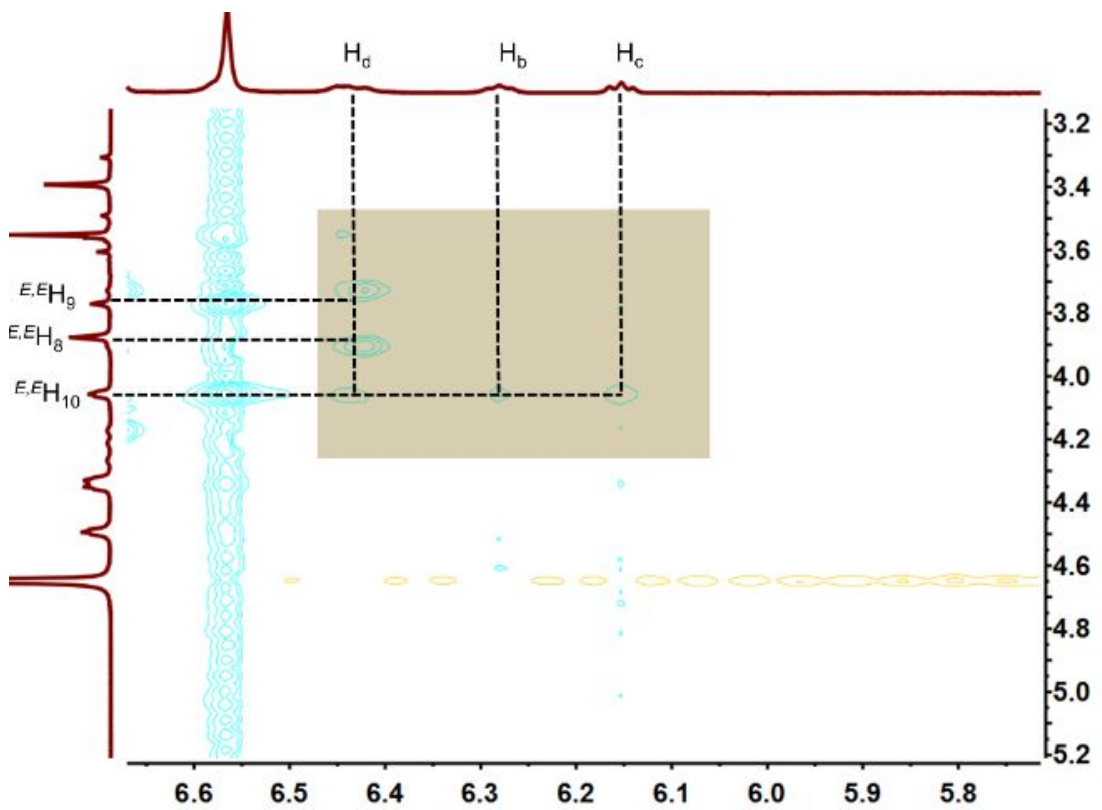

Figure S18 2D NOESY NMR spectrum (600 MHz, $\left.\mathrm{D}_{2} \mathrm{O}, 298 \mathrm{~K}\right)$ of $1.00 \mathrm{mM} E, E-1$ and $1.00 \mathrm{mM}$ PHE.

To determine the stoichiometry for the complexation between $E, E-1$ and ANT or PHE, ${ }^{1} \mathrm{H}$ NMR titration was done with solutions which had a constant concentration of the guest molecule $(1.00 \mathrm{mM})$ and varying concentrations of $E, E-1$. By a mole ratio plot, $1: 1$ stoichiometry was obtained for the complexation between $E, E-1$ and ANT or PHE.

(a)

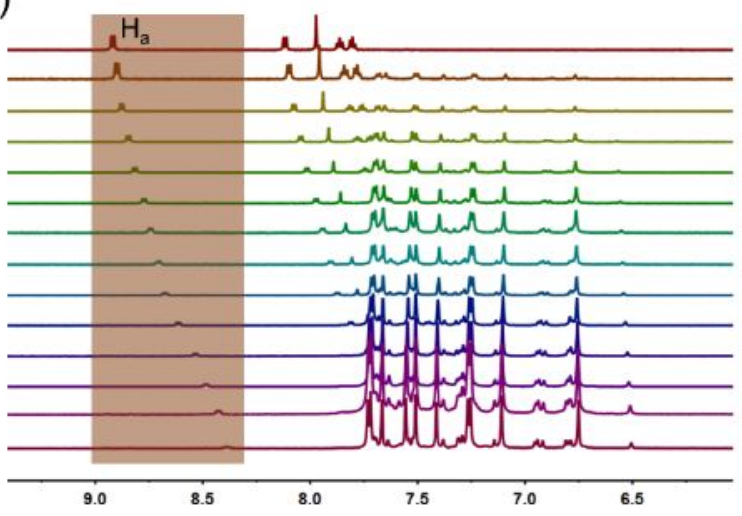

(b)

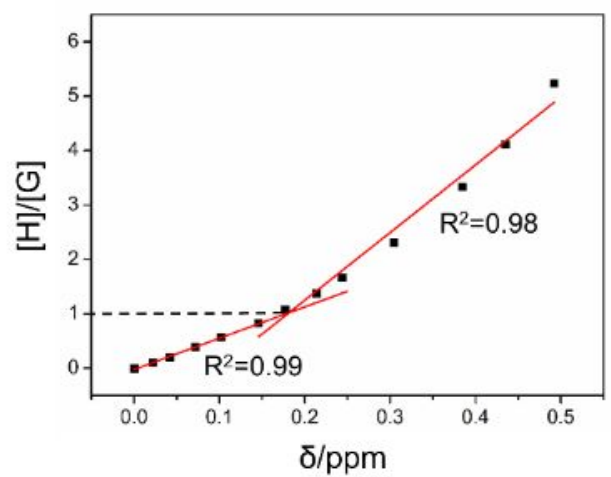

Figure S19 (a) Partial ${ }^{1} \mathrm{H}$ NMR spectra $\left(600 \mathrm{MHz}, \mathrm{D}_{2} \mathrm{O} / \mathrm{DMSO}-d_{6}=1: 1,298 \mathrm{~K}\right)$ of PHE at a concentration of $1.00 \mathrm{mM}$ upon addition of various concentrations of $E, E-\mathbf{1}$ : $0.00 \mathrm{mM}, 0.10 \mathrm{mM}, 0.20 \mathrm{mM}, 0.38 \mathrm{mM}, 0.57 \mathrm{mM}, 0.82 \mathrm{mM}, 1.07 \mathrm{mM}, 1.38 \mathrm{mM}$, 
$1.67 \mathrm{mM}, 2.31 \mathrm{mM}, 3.33 \mathrm{mM}, 4.11 \mathrm{mM}, 5.24 \mathrm{mM}$ and $6.31 \mathrm{mM}$ from top to bottom.

(b) Mole ratio plot for E,E-1 and PHE, indicating a $1: 1$ stoichiometry.

(a)

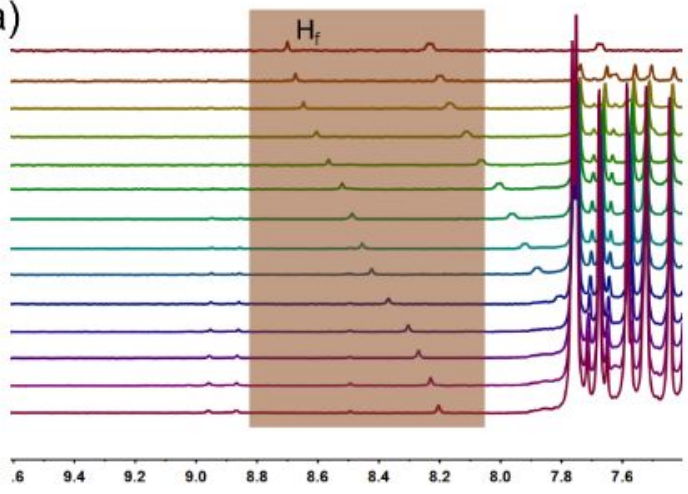

(b)

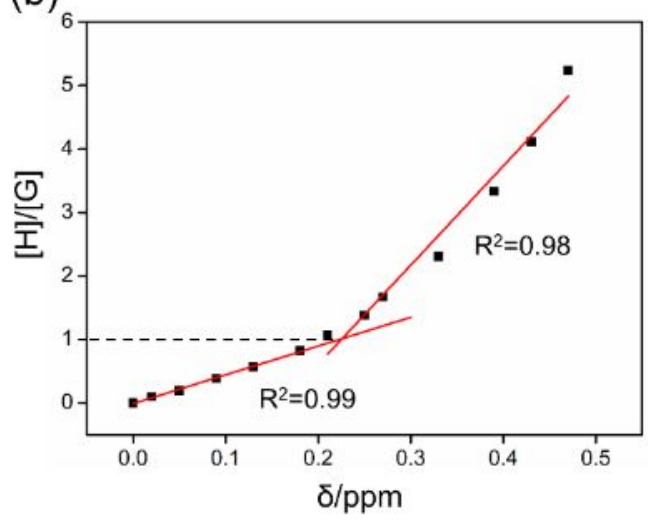

Figure S20 (a) Partial ${ }^{1} \mathrm{H}$ NMR spectra $\left(600 \mathrm{MHz}, \mathrm{D}_{2} \mathrm{O} / \mathrm{DMSO}-d_{6}=1: 1,298 \mathrm{~K}\right)$ of ANT at a concentration of $1.00 \mathrm{mM}$ upon addition of various concentrations of $E, E-\mathbf{1}$ : $0.00 \mathrm{mM}, 0.10 \mathrm{mM}, 0.20 \mathrm{mM}, 0.38 \mathrm{mM}, 0.57 \mathrm{mM}, 0.82 \mathrm{mM}, 1.07 \mathrm{mM}, 1.38 \mathrm{mM}$, $1.67 \mathrm{mM}, 2.31 \mathrm{mM}, 3.33 \mathrm{mM}, 4.11 \mathrm{mM}, 5.24 \mathrm{mM}$ and $6.31 \mathrm{mM}$ from top to bottom. (b) Mole ratio plot for $E, E-1$ and ANT, indicating a $1: 1$ stoichiometry.

(a)

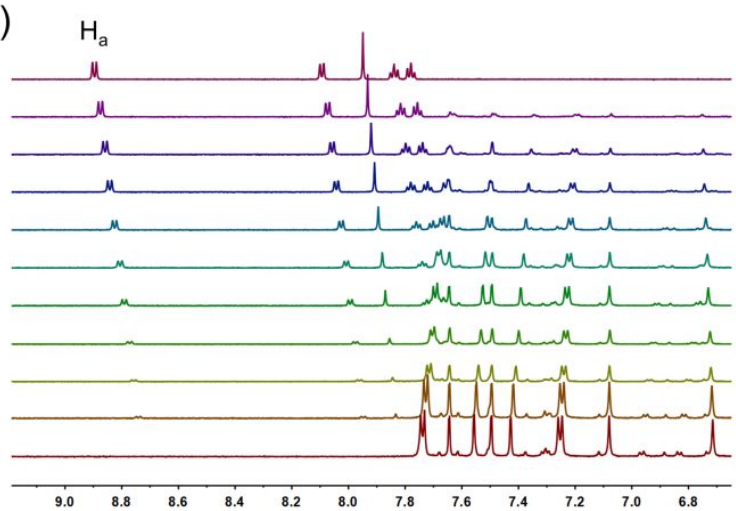

(b)

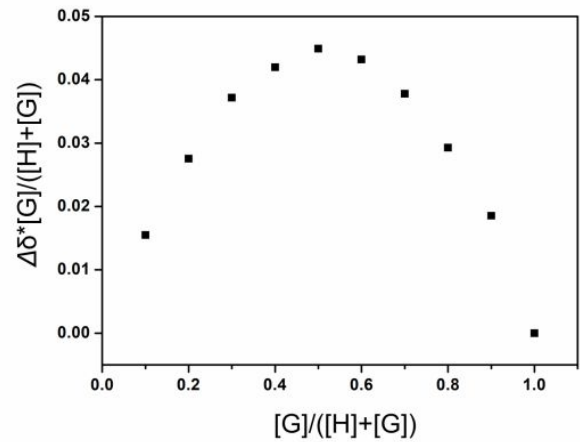

Figure S21 (a) Partial ${ }^{1} \mathrm{H}$ NMR spectra $\left(600 \mathrm{MHz}, \mathrm{D}_{2} \mathrm{O} / \mathrm{DMSO}-d_{6}=1 / 1, v / v, 298 \mathrm{~K}\right)$ of PHE and $E, E-\mathbf{1}([\mathrm{H}]+[\mathrm{G}]=1.0 \mathrm{mM})$ at different molar ratios. (b) Job plot for $1: 1$ complexation of $\mathrm{H}$ with $\mathrm{G}$. 
(a)

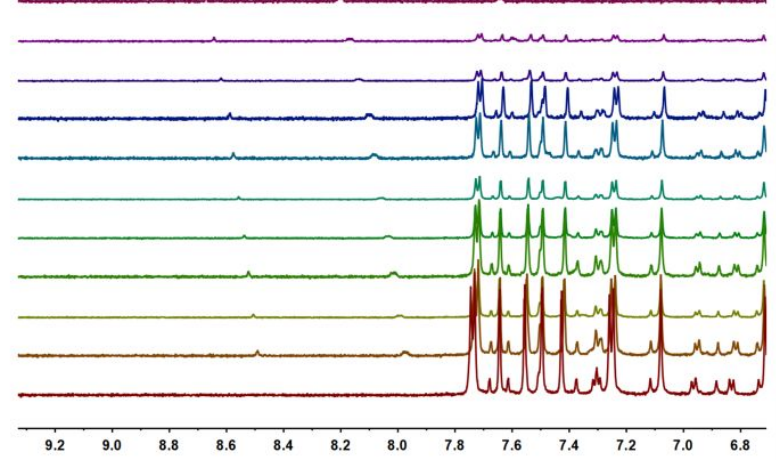

(b)

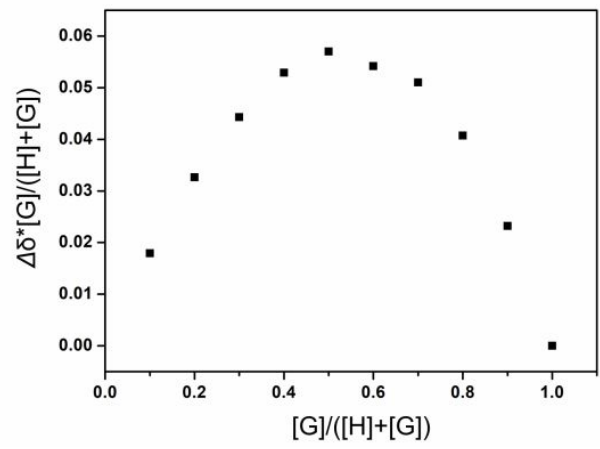

Figure S22 (a) Partial ${ }^{1} \mathrm{H}$ NMR spectra $\left(600 \mathrm{MHz}, \mathrm{D}_{2} \mathrm{O} / \mathrm{DMSO}-d_{6}=1: 1, v / v, 298 \mathrm{~K}\right)$ of ANT and $E, E-1([\mathrm{H}]+[\mathrm{G}]=1.0 \mathrm{mM})$ at different molar ratios. (b) Job plot for 1 : 1 complexation of $\mathrm{H}$ with $\mathrm{G}$.

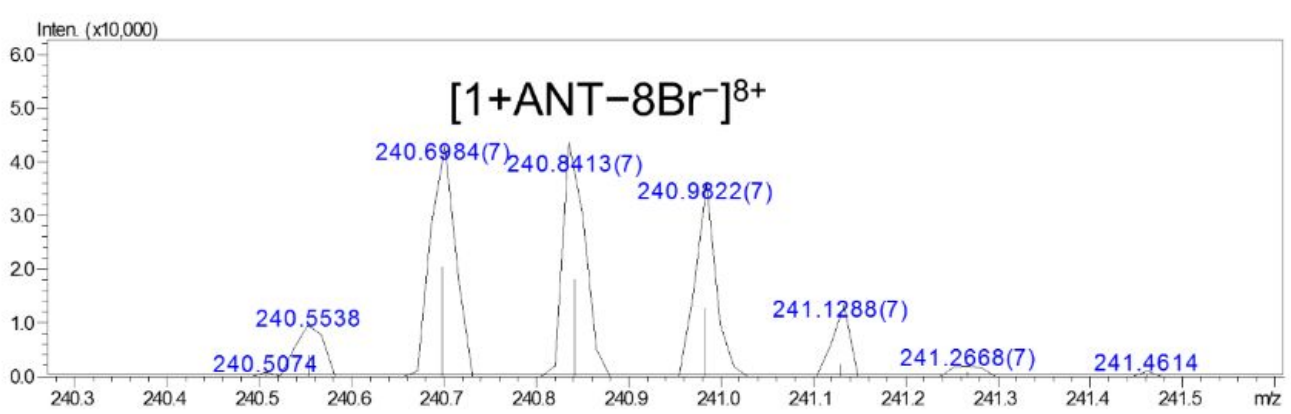

Figure S23 HRMS of the sample of ANT dissolved in an aqueous solution of $E, E-1$.

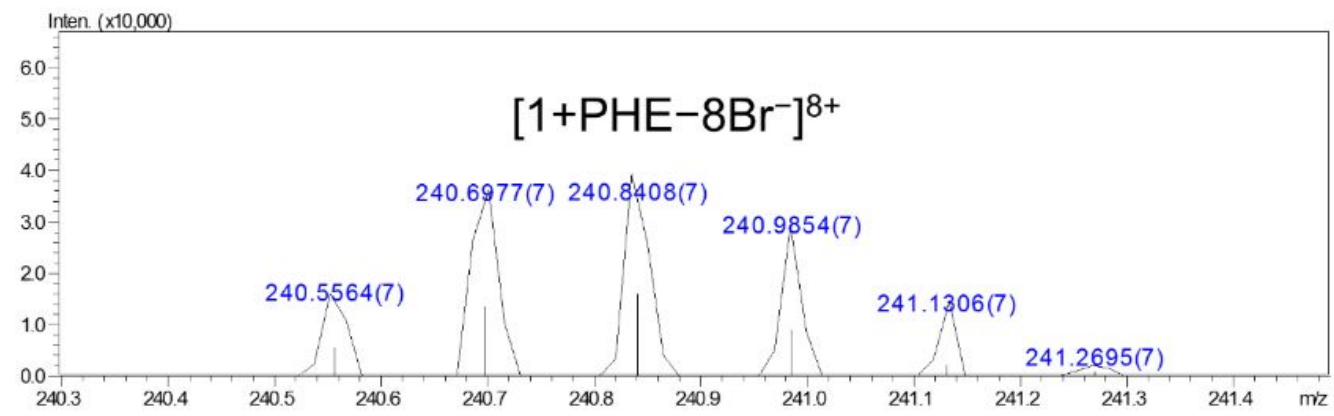

Figure S24 HRMS of the sample of PHE dissolved in an aqueous solution of $E, E-1$.

We measured the association constant $K_{\mathrm{a}}$ between $E, E-\mathbf{1}$ and ANT or PHE in water by the practical separation of ANT or PHE using Eq. S1. ANT or PHE (10.0 mg), E,E-1 (145 mg) and $15 \mathrm{~mL}$ of water were added to a $25 \mathrm{~mL}$ round-bottom flask, and the suspension was stirred at room temperature for 24 hours. After that, the mixture was 
filtered and the solution was irradiated by UV light $(365 \mathrm{~nm})$ for 2 hours. Then, the solution was extracted with $\mathrm{CHCl}_{3}$, and the organic layer was concentrated by rotary evaporation. ${ }^{1} \mathrm{H}$ NMR spectra was carried out to detect the content of ANT (Figure S24) or PHE (Figure S23) using $2 \mu \mathrm{L}$ of 1,1,2,2-tetrachloroethane as the internal standard.

The $K_{\mathrm{a}}$ was calculated by the following formula:

$$
\begin{aligned}
& \mathrm{H}+\mathrm{G} \longrightarrow \mathrm{HG} \\
& \quad K_{\mathrm{a}}=[\mathrm{HG}] /([\mathrm{H}][\mathrm{G}]) \ldots \ldots \ldots \ldots \ldots \ldots \ldots \ldots(\text { Eq. } \mathrm{S} 1)
\end{aligned}
$$

$[\mathrm{H}]$ is the concentration of dissociative $E, E-\mathbf{1}$ at equilibrium, $[\mathrm{G}]$ is the concentration of the dissociative guest at equilibrium, and $[\mathrm{HG}]$ is the concentration of the host-guest complex at equilibrium.
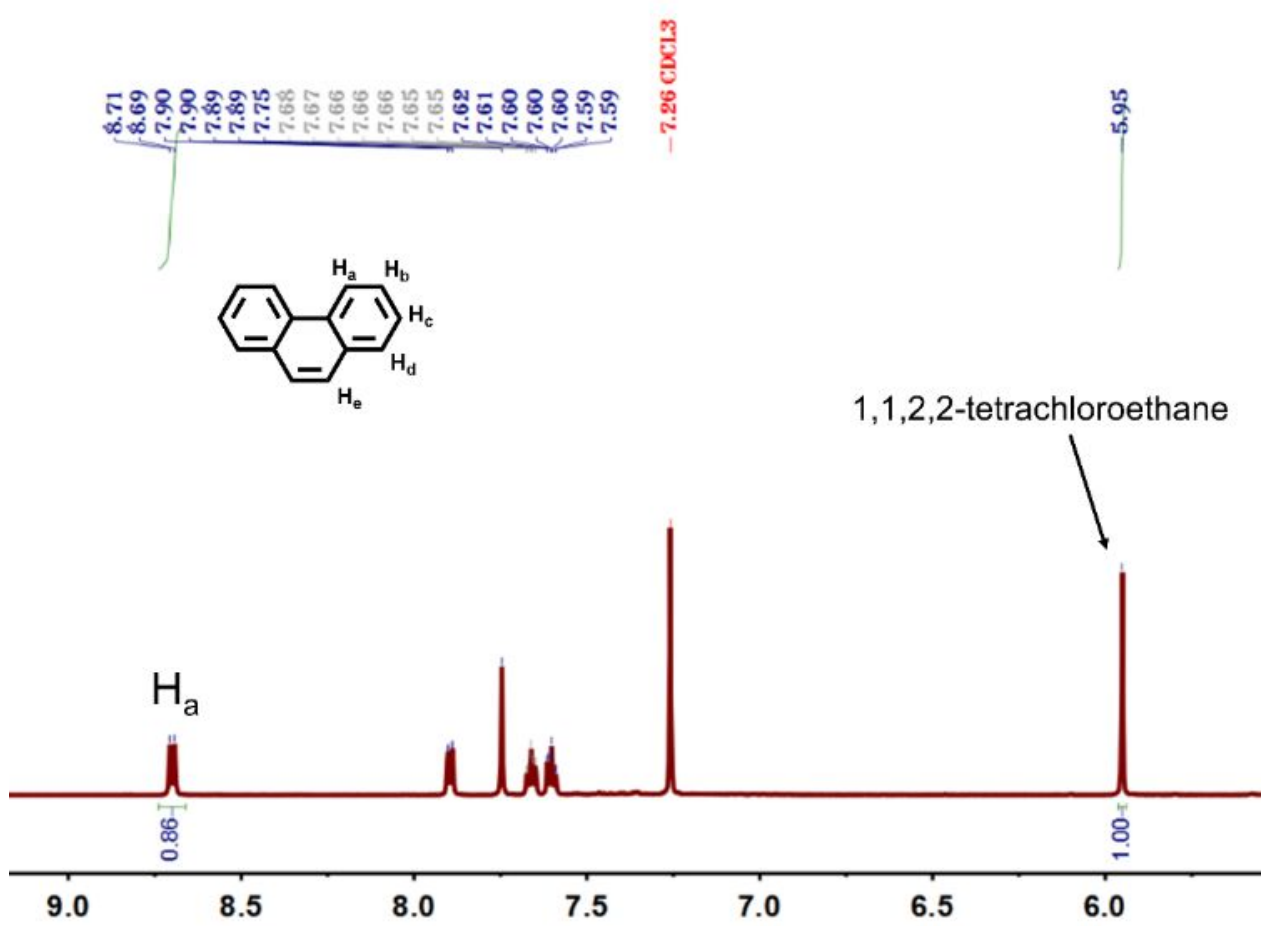

Figure S25 Partial ${ }^{1} \mathrm{H}$ NMR spectrum $\left(600 \mathrm{MHz}, \mathrm{CDCl}_{3}, 298 \mathrm{~K}\right)$ of PHE and 1,1,2,2-tetrachloroethane. 


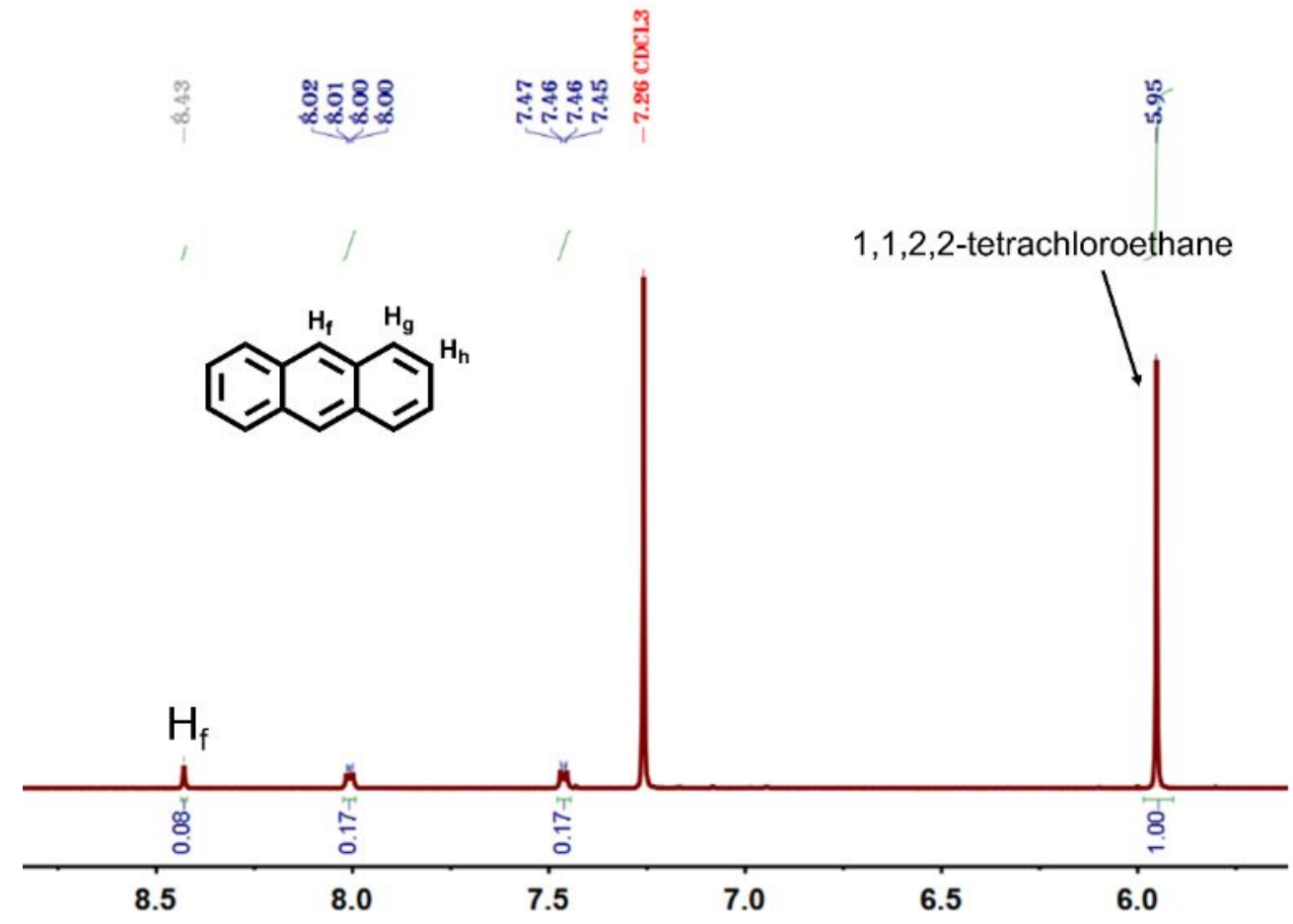

Figure S26 Partial ${ }^{1} \mathrm{H}$ NMR spectrum $\left(600 \mathrm{MHz}, \mathrm{CDCl}_{3}, 298 \mathrm{~K}\right)$ of ANT and 1,1,2,2-tetrachloroethane.

The solubility $(298 \mathrm{~K})$ of PHE in water is $8.1 \times 10^{-6} \mathrm{M}^{[\mathrm{S} 12]}$. We deduced that the binding constant of $E, E-1$ and PHE was $4.5 \times 10^{4} \mathrm{M}^{-1}$.

The solubility $(298 \mathrm{~K})$ of ANT in water is $3.9 \times 10^{-7} \mathrm{M}^{[\mathrm{S} 12]}$. We deduced that the binding constant of $E, E-1$ and ANT was $6.7 \times 10^{4} \mathrm{M}^{-1}$.
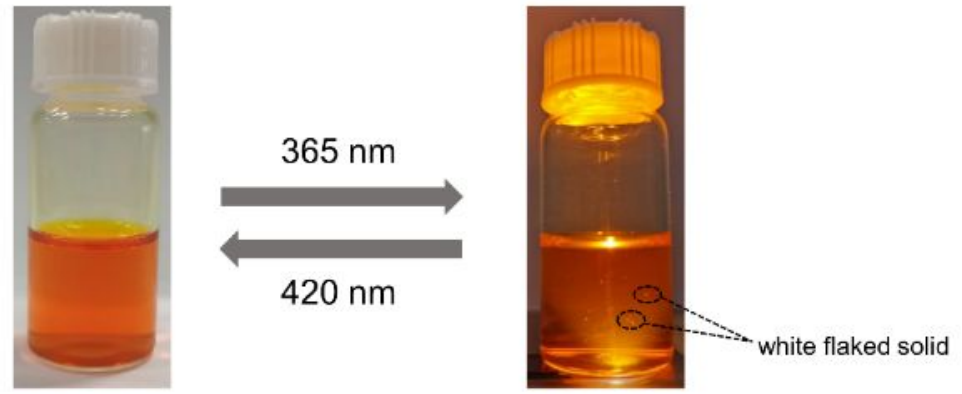

Figure S27 Photographs showing the change of a mixture of $E, E-1$ and PHE in water after UV light (365 $\mathrm{nm})$ and visible light $(420 \mathrm{~nm})$ irradiation, respectively. 


\section{Selective separation of PHE from aromatic isomer mixtures and cycling experiments}

The separation experiment was carried out as follows:

ANT (10.0 mg), PHE (10.0 mg), E,E-1 (300 mg) and $15 \mathrm{~mL}$ of water were added to a $25 \mathrm{~mL}$ round-bottom flask, and the suspension was stirred at room temperature for 2 hours. After that, the mixture was filtered. The solution containing E,E-1 and guest molecules was irradiated by UV light $(365 \mathrm{~nm})$ for 1 hour in order to release guest molecules and a white flaky solid formed during this process. Then, the solid-liquid separation was achieved by centrifugation and filtration. The solution was transferred to next separation experiment after visible light $(420 \mathrm{~nm})$ irradiation for 1 hour. The solid was dried in a vacuum drying chamber, and ${ }^{1} \mathrm{H}$ NMR was carried out to detect the composition of the solid using $1 \mu \mathrm{L}$ of 1,1,2,2-tetrachloroethane as the internal standard.

1,1,2,2-tetrachloroethane

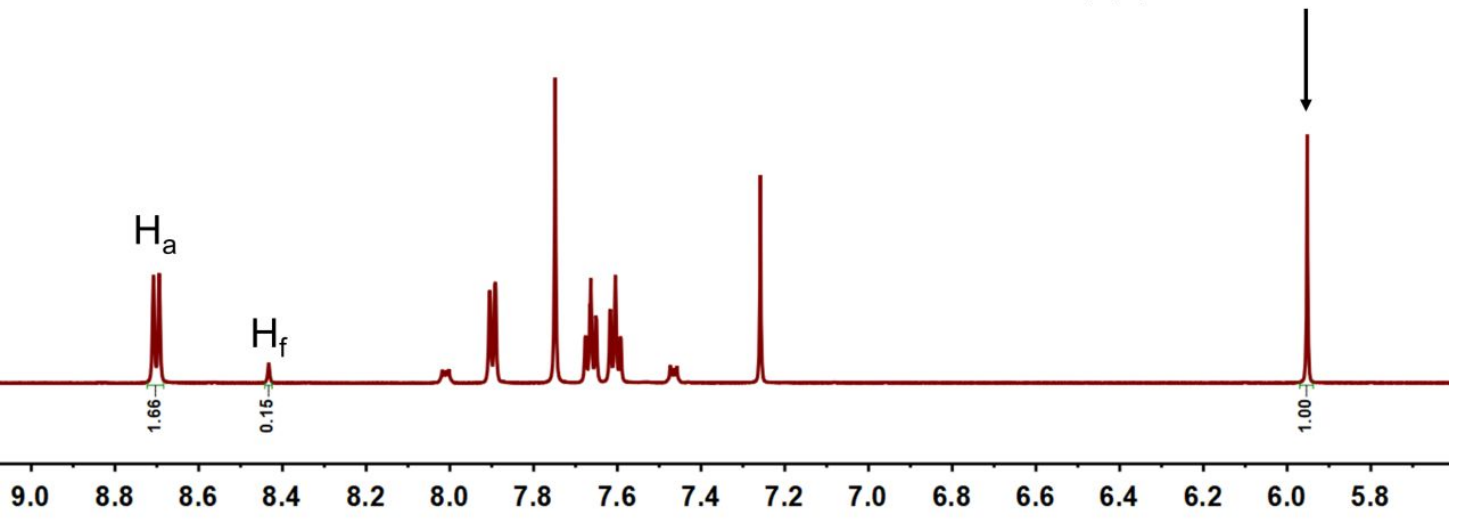

Figure S28 Partial ${ }^{1} \mathrm{H}$ NMR spectra $\left(600 \mathrm{MHz}, \mathrm{CDCl}_{3}, 298 \mathrm{~K}\right)$ of the solid phase after the first separation, indicating $3.09 \mathrm{mg}$ of solid product was obtained and the purity for PHE was $91.7 \%$. 


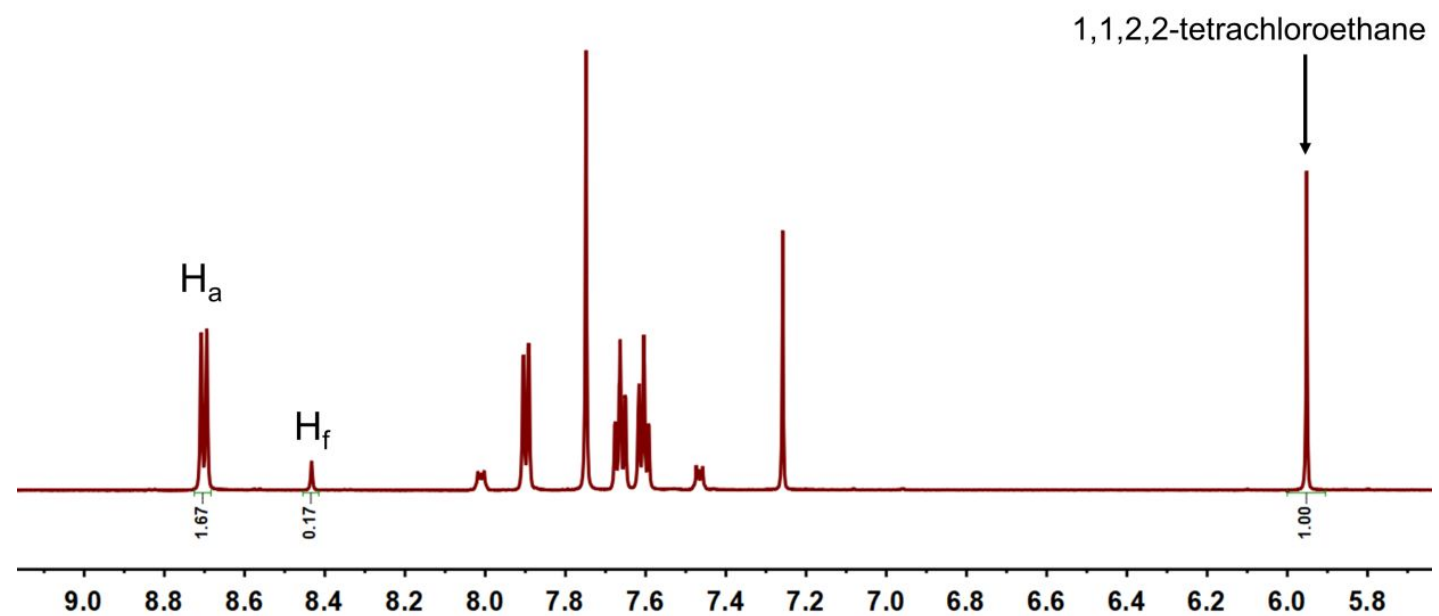

Figure S29 Partial ${ }^{1} \mathrm{H}$ NMR spectra $\left(600 \mathrm{MHz}, \mathrm{CDCl}_{3}, 298 \mathrm{~K}\right)$ of the solid phase after the second separation, indicating $3.14 \mathrm{mg}$ of solid product was obtained and the purity for PHE was $90.7 \%$.

1,1,2,2-tetrachloroethane

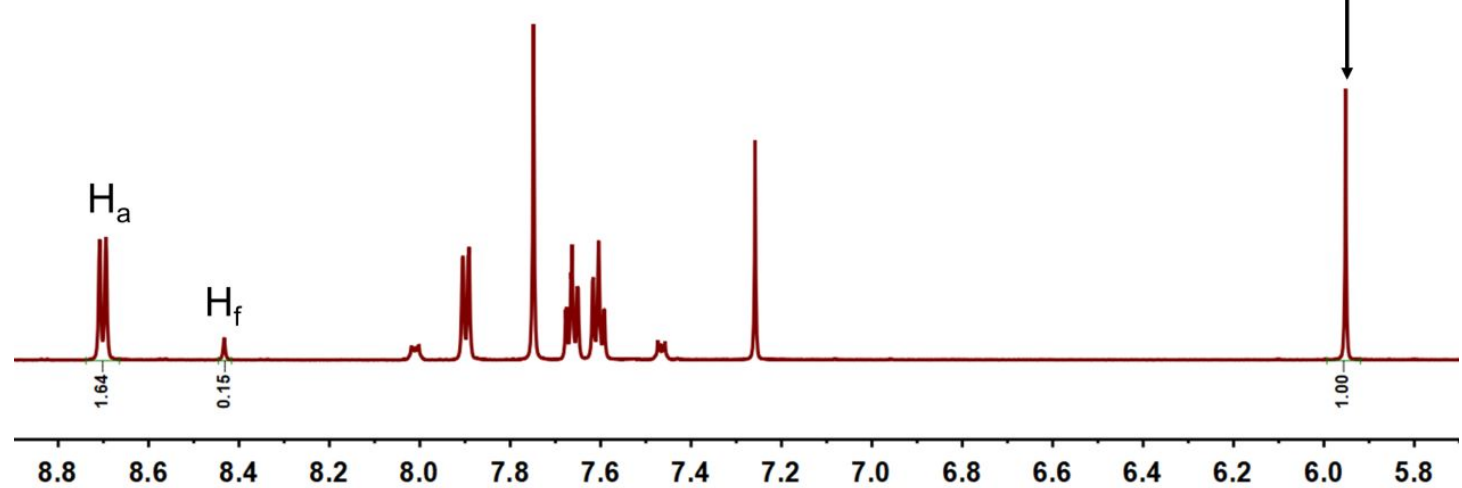

Figure S30 Partial ${ }^{1} \mathrm{H}$ NMR spectra $\left(600 \mathrm{MHz}, \mathrm{CDCl}_{3}, 298 \mathrm{~K}\right)$ of the solid phase after the third separation, indicating $3.05 \mathrm{mg}$ of solid product was obtained and the purity for PHE was $91.6 \%$. 


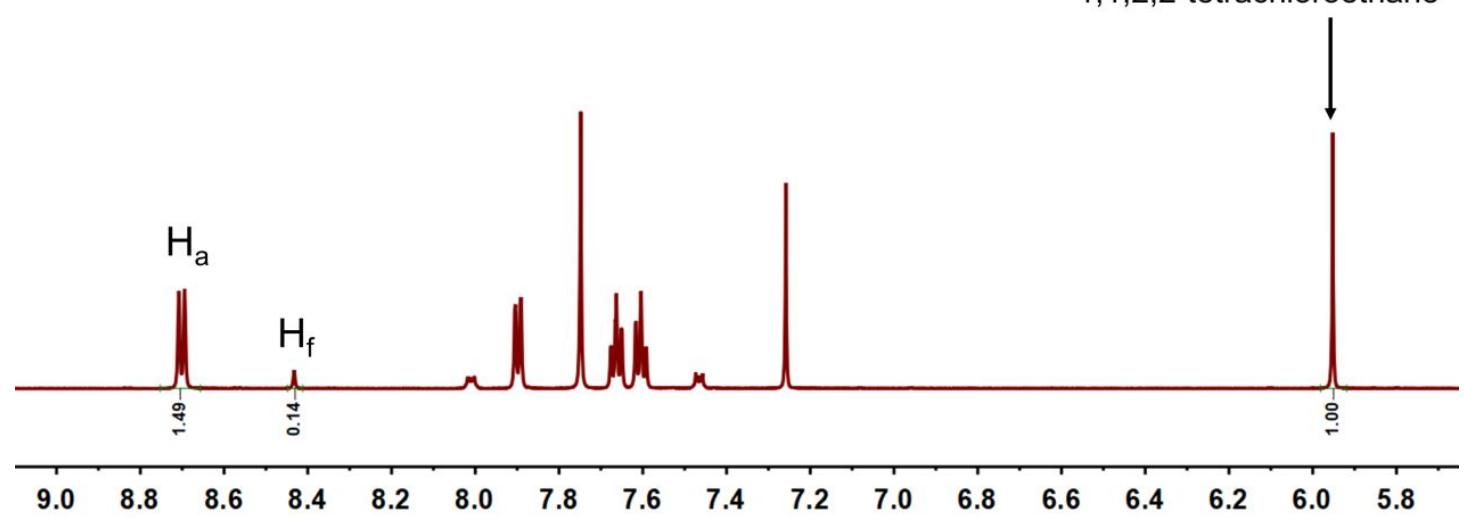

Figure S31 Partial ${ }^{1} \mathrm{H}$ NMR spectra $\left(600 \mathrm{MHz}, \mathrm{CDCl}_{3}, 298 \mathrm{~K}\right)$ of the solid phase after the forth separation, indicating $2.78 \mathrm{mg}$ of solid product was obtained and the purity for PHE was $91.4 \%$.

1,1,2,2-tetrachloroethane

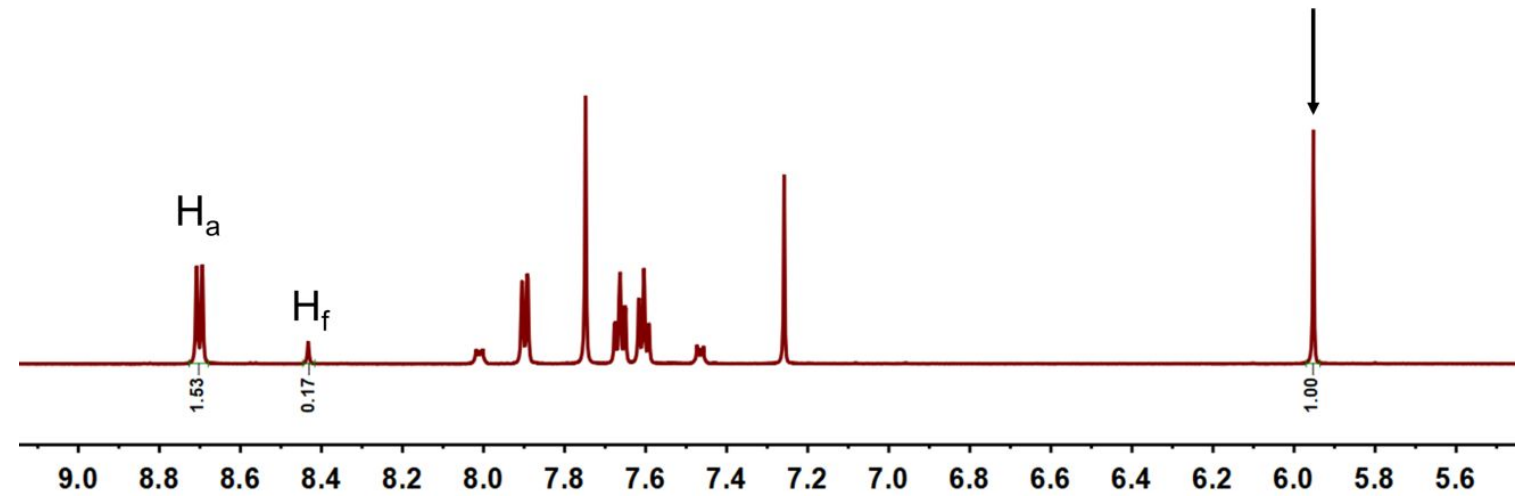

Figure S32 Partial ${ }^{1} \mathrm{H}$ NMR spectra $\left(600 \mathrm{MHz}, \mathrm{CDCl}_{3}, 298 \mathrm{~K}\right)$ of the solid phase after the fifth separation, indicating $2.90 \mathrm{mg}$ of solid product was obtained and the purity for PHE was $90.0 \%$.

From the results of the separation, the average weight for the solid product is $2.99 \mathrm{mg}$ and the average purity for PHE is $91.1 \%$ in five extraction cycles. 

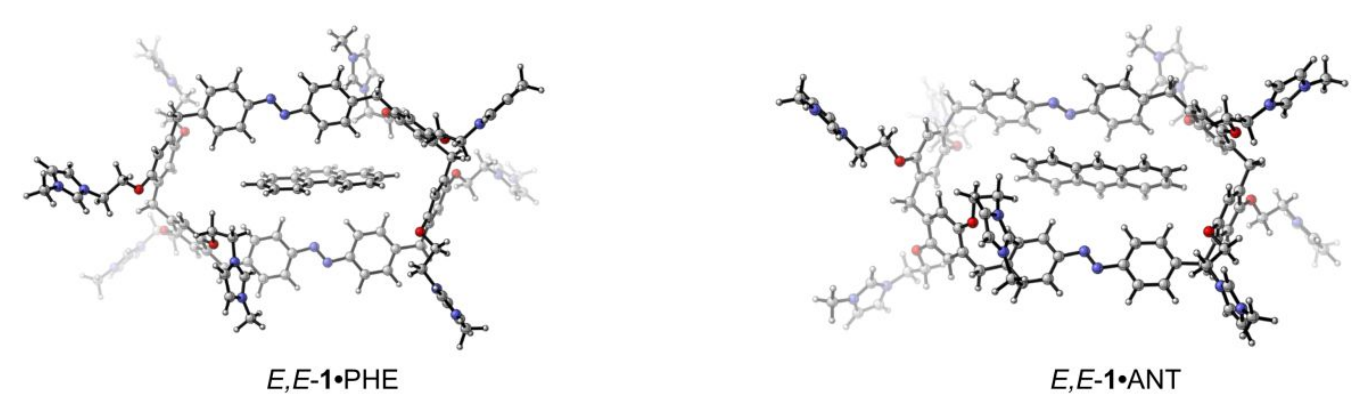

Figure S33 The DFT-optimized structures of $E, E-\mathbf{1} \bullet P H E$ and $E, E-\mathbf{1} \bullet A N T$.

\section{References}

S1. L. Wu, Y. He, X. Tang. Bioconjugate Chem. 2015, 26, 1070-1079.

S2. Gaussian 09, Revision D. 01, M. J. Frisch, G. W. Trucks, H. B. Schlegel, G. E. Scuseria, M. A. Robb, J. R. Cheeseman, G. Scalmani, V. Barone, B. Mennucci, G. A. Petersson, H. Nakatsuji, M. Caricato, X. Li, H. P. Hratchian, A. F. Izmaylov, J. Bloino, G. Zheng, J. L. Sonnenberg, M. Hada, M. Ehara, K. Toyota, R. Fukuda, J. Hasegawa, M. Ishida, T. Nakajima, Y. Honda, O. Kitao, H. Nakai, T. Vreven, J. A. Montgomery, Jr., J. E. Peralta, F. Ogliaro, M. Bearpark, J. J. Heyd, E. Brothers, K. N. Kudin, V. N. Staroverov, T. Keith, R. Kobayashi, J. Normand, K. Raghavachari, A. Rendell, J. C. Burant, S. S. Iyengar, J. Tomasi, M. Cossi, N. Rega, J. M. Millam, M. Klene, J. E. Knox, J. B. Cross, V. Bakken, C. Adamo, J. Jaramillo, R. Gomperts, R. E. Stratmann, O. Yazyev, A. J. Austin, R. Cammi, C. Pomelli, J. W. Ochterski, R. L. Martin, K. Morokuma, V. G. Zakrzewski, G. A. Voth, P. Salvador, J. J. Dannenberg, S. Dapprich, A. D. Daniels, O. Farkas, J. B. Foresman, J. V. Ortiz, J. Cioslowski, and D. J. Fox, Gaussian, Inc., Wallingford CT, 2013.

S3. C. Lee, W. Yang, R. G. Parr, Phys. Rev. B. 1988, 37, 785-789.

S4. A. D. Becke, J. Chem. Phys. 1993, 98, 5648-5652.

S5. P. J. Stephens, F. J. Devlin, C. F. Chabalowski, M. J. Frisch, J. Phys. Chem. 1994, 98, 11623-11627. 
S6. S. Grimme, J. Antony, S. Ehrlich, H. Krieg, J. Chem. Phys. 2010, 132, 154104.

S7. S. Grimme, S. Ehrlich, L. Goerigk, J. Comput. Chem. 2011, 32, 1456-1465.

S8. F. Weigend, R. Ahlrichs, Phys. Chem. Chem. Phys. 2005, 7, 3297-3305.

S9. F. Weigend, Phys. Chem. Chem. Phys. 2006, 8, 1057-1065.

S10. A. V. Marenich, C. J. Cramer, D. G. Truhlar, J. Phys. Chem. B 2009, 113, $6378-6396$.

S11. C. Y. Legault, CYLview, 1.0b, Université de Sherbrooke 2009, (http://www.cylview.org).

S12. X. Wang, M. L. Brusseau. Environ. Sci. Technol. 1995, 29, 2346-2351.

Table S2. Zero-point correction ( $Z P E)$, thermal correction to enthalpy $(T C H)$, thermal correction to Gibbs free energy $(T C G)$, energies $(E)$, enthalpies $(H)$, and Gibbs free energies $(G)$ (in Hartree) of the structures for all the figures calculated at the B3LYP-D3(BJ)/def2-TZVP-SMD(water)//B3LYP-D3(BJ)/def2-SVP level of theory.

\begin{tabular}{|c|c|c|c|c|c|c|c|}
\hline Structures & $Z P E$ & $T C H$ & $T C G$ & $E$ & $\boldsymbol{H}$ & $G$ & $\begin{array}{l}\text { Imaginary } \\
\text { Frequency }\end{array}$ \\
\hline E,E-1 & 2.066690 & 2.190596 & 1.866728 & -5654.909586 & -5652.71899 & -5653.042858 & \\
\hline E,Z-1 & 2.066893 & 2.190766 & 1.864416 & -5654.874141 & -5652.683375 & -5653.009725 & \\
\hline Z,Z-1 & 2.067108 & 2.190368 & 1.869516 & -5654.858609 & -5652.668241 & -5652.989093 & \\
\hline EE-1.PHE & 2.264298 & 2.399404 & 2.050845 & -6194.736069 & -6192.336665 & -6192.685224 & \\
\hline EE-1·ANT & 2.264586 & 2.399254 & 2.055278 & -6194.72677 & -6192.327516 & -6192.671492 & \\
\hline Phenanthrene & 0.195302 & 0.205568 & 0.161201 & -539.788535 & -539.582967 & -539.627334 & \\
\hline Anthracene & 0.194804 & 0.205081 & 0.160765 & -539.77941 & -539.574329 & -539.618645 & \\
\hline
\end{tabular}

\section{Cartesian coordinates of the calculated species}

anthracene

01

$\begin{array}{lrrr}\text { C } & 3.66414700 & 0.71358100 & 0.00009100 \\ \text { C } & 2.48118800 & 1.40838400 & 0.00011400 \\ \text { C } & 1.22491100 & 0.72317700 & -0.00011400 \\ \text { C } & 1.22491100 & -0.72317700 & -0.00011400\end{array}$




\begin{tabular}{|c|c|c|c|}
\hline $\mathrm{C}$ & 2.48118800 & -1.40838400 & 0.00011400 \\
\hline $\mathrm{C}$ & 3.66414700 & -0.71358100 & 0.00009100 \\
\hline $\mathrm{C}$ & 0.00000000 & 1.40508400 & -0.00019200 \\
\hline $\mathrm{C}$ & 0.00000000 & -1.40508400 & -0.00019200 \\
\hline $\mathrm{C}$ & -1.22491100 & -0.72317700 & -0.00011400 \\
\hline $\mathrm{C}$ & -1.22491100 & 0.72317700 & -0.00011400 \\
\hline $\mathrm{C}$ & -2.48118800 & 1.40838400 & 0.00011400 \\
\hline $\mathrm{H}$ & -2.47855100 & 2.50146400 & -0.00004700 \\
\hline $\mathrm{C}$ & -3.66414700 & 0.71358100 & 0.00009100 \\
\hline $\mathrm{C}$ & -3.66414700 & -0.71358100 & 0.00009100 \\
\hline $\mathrm{C}$ & -2.48118800 & -1.40838400 & 0.00011400 \\
\hline $\mathrm{H}$ & 0.00000000 & 2.49863800 & -0.00002200 \\
\hline $\mathrm{H}$ & 4.61583100 & 1.25028300 & 0.00009200 \\
\hline $\mathrm{H}$ & 2.47855100 & 2.50146400 & -0.00004700 \\
\hline $\mathrm{H}$ & 2.47855100 & -2.50146400 & -0.00004700 \\
\hline $\mathrm{H}$ & 4.61583100 & -1.25028300 & 0.00009200 \\
\hline $\mathrm{H}$ & 0.00000000 & -2.49863800 & -0.00002200 \\
\hline $\mathrm{H}$ & -4.61583100 & 1.25028300 & 0.00009200 \\
\hline $\mathrm{H}$ & -4.61583100 & -1.25028300 & 0.00009200 \\
\hline $\mathrm{H}$ & -2.47855100 & -2.50146400 & -0.00004700 \\
\hline
\end{tabular}

\section{E,E-1}

81

$\begin{array}{llrr}\mathrm{O} & -9.03812000 & 0.78743600 & -3.44225600 \\ \mathrm{O} & -6.07518900 & -3.36284100 & -1.27528700 \\ \mathrm{O} & -6.59160800 & 4.17252000 & 0.71433800 \\ \mathrm{O} & -9.55624200 & -0.29143200 & 2.13978200 \\ \mathrm{~N} & 0.74422900 & -3.69075000 & -3.38257900 \\ \mathrm{~N} & 0.05421500 & -2.80830500 & -2.82159400 \\ \mathrm{C} & 2.89215200 & -4.58067800 & -3.90160600\end{array}$




\begin{tabular}{|c|c|c|c|}
\hline $\mathrm{H}$ & 2.35257300 & -5.38309700 & -4.40788700 \\
\hline $\mathrm{C}$ & 4.28461900 & -4.52181400 & -3.91794000 \\
\hline $\mathrm{H}$ & 4.84539800 & -5.28996700 & -4.45862700 \\
\hline $\mathrm{C}$ & 4.97136200 & -3.46303300 & -3.30293600 \\
\hline $\mathrm{C}$ & 4.21902300 & -2.48061300 & -2.63372200 \\
\hline $\mathrm{H}$ & 4.73650400 & -1.65413800 & -2.14081300 \\
\hline $\mathrm{C}$ & 2.82702600 & -2.53158000 & -2.60833900 \\
\hline $\mathrm{H}$ & 2.23993200 & -1.75775500 & -2.11224400 \\
\hline $\mathrm{C}$ & 2.14678600 & -3.57901300 & -3.25947400 \\
\hline $\mathrm{C}$ & -1.34028700 & -2.89592700 & -3.06476500 \\
\hline $\mathrm{C}$ & -1.92831000 & -3.79579700 & -3.97533800 \\
\hline $\mathrm{H}$ & -1.28425800 & -4.49628100 & -4.50776200 \\
\hline $\mathrm{C}$ & -3.29842700 & -3.74569300 & -4.21035200 \\
\hline $\mathrm{H}$ & -3.73464300 & -4.41270900 & -4.96149500 \\
\hline $\mathrm{C}$ & -4.12980900 & -2.81294700 & -3.55467300 \\
\hline $\mathrm{C}$ & -3.53860000 & -1.93744400 & -2.63518900 \\
\hline $\mathrm{H}$ & -4.15621900 & -1.20127400 & -2.11649900 \\
\hline $\mathrm{C}$ & -2.16143600 & -1.97508800 & -2.39677800 \\
\hline $\mathrm{H}$ & -1.69058500 & -1.27622700 & -1.70237400 \\
\hline $\mathrm{C}$ & -5.60222000 & -2.76666300 & -3.93666500 \\
\hline $\mathrm{H}$ & -5.98041500 & -3.80433600 & -3.96832200 \\
\hline $\mathrm{H}$ & -5.66961600 & -2.40570400 & -4.97603200 \\
\hline $\mathrm{C}$ & -6.54497100 & -1.94881200 & -3.08573800 \\
\hline $\mathrm{C}$ & -7.28239800 & -0.89692400 & -3.63566300 \\
\hline $\mathrm{H}$ & -7.11560300 & -0.66036500 & -4.68586900 \\
\hline $\mathrm{C}$ & -8.23398500 & -0.18769000 & -2.88654200 \\
\hline $\mathrm{C}$ & -8.41592700 & -0.47913300 & -1.52213800 \\
\hline $\mathrm{C}$ & -7.68322200 & -1.54344600 & -0.97062400 \\
\hline $\mathrm{H}$ & -7.84378700 & -1.77996800 & 0.08240100 \\
\hline $\mathrm{C}$ & -6.78215000 & -2.28464800 & -1.74014700 \\
\hline
\end{tabular}




\begin{tabular}{|c|c|c|c|}
\hline $\mathrm{C}$ & -9.36888600 & 0.32307700 & -0.64923700 \\
\hline $\mathrm{H}$ & -9.93895100 & 1.01088700 & -1.28685200 \\
\hline $\mathrm{H}$ & -10.08836600 & -0.35888000 & -0.17575800 \\
\hline $\mathrm{C}$ & -8.66619300 & 1.12306600 & 0.43543300 \\
\hline $\mathrm{C}$ & -7.92065900 & 2.26516800 & 0.10889600 \\
\hline $\mathrm{H}$ & -7.86232400 & 2.59188500 & -0.93185400 \\
\hline $\mathrm{C}$ & -7.26370800 & 3.02161800 & 1.08106900 \\
\hline $\mathrm{C}$ & -7.25805200 & 2.61042900 & 2.42558900 \\
\hline $\mathrm{C}$ & -8.02050200 & 1.48408100 & 2.76051500 \\
\hline $\mathrm{H}$ & -8.03842800 & 1.16186100 & 3.80293700 \\
\hline $\mathrm{C}$ & -8.74880900 & 0.77157600 & 1.79717800 \\
\hline $\mathrm{C}$ & -8.70965700 & 1.29354600 & -4.72400400 \\
\hline $\mathrm{H}$ & -8.69436300 & 0.49370900 & -5.48490900 \\
\hline $\mathrm{H}$ & -7.70778800 & 1.75820600 & -4.70334200 \\
\hline $\mathrm{C}$ & -6.35528500 & -3.91748700 & -0.01513700 \\
\hline $\mathrm{H}$ & -7.41325900 & -4.23301800 & 0.05162100 \\
\hline $\mathrm{H}$ & -6.15964800 & -3.19902400 & 0.80159800 \\
\hline $\mathrm{C}$ & -7.28037100 & 5.38644200 & 0.94428300 \\
\hline $\mathrm{H}$ & -7.97625600 & 5.61053300 & 0.11367700 \\
\hline $\mathrm{H}$ & -7.88103400 & 5.33749400 & 1.86933900 \\
\hline $\mathrm{C}$ & -10.17961700 & -0.28461200 & 3.41023200 \\
\hline $\mathrm{H}$ & -10.45679600 & 0.74447700 & 3.69906000 \\
\hline $\mathrm{H}$ & -9.51127400 & -0.68555200 & 4.19355400 \\
\hline $\mathrm{C}$ & -6.47861200 & 3.36016400 & 3.48302300 \\
\hline $\mathrm{H}$ & -6.66811200 & 2.87661300 & 4.45609400 \\
\hline $\mathrm{H}$ & -6.88314300 & 4.37998000 & 3.59627200 \\
\hline $\mathrm{O}$ & 9.03812000 & -0.78743600 & 3.44225600 \\
\hline $\mathrm{O}$ & 6.07518900 & 3.36284100 & 1.27528700 \\
\hline $\mathrm{O}$ & 6.59160800 & -4.17252000 & -0.71433800 \\
\hline $\mathrm{O}$ & 9.55624200 & 0.29143200 & -2.13978200 \\
\hline
\end{tabular}




\begin{tabular}{|c|c|c|c|}
\hline $\mathrm{N}$ & -0.74422900 & 3.69075000 & 3.38257900 \\
\hline $\mathrm{N}$ & -0.05421500 & 2.80830500 & 2.82159400 \\
\hline $\mathrm{C}$ & -2.89215200 & 4.58067800 & 3.90160600 \\
\hline $\mathrm{H}$ & -2.35257300 & 5.38309700 & 4.40788700 \\
\hline $\mathrm{C}$ & -4.28461900 & 4.52181400 & 3.91794000 \\
\hline $\mathrm{H}$ & -4.84539800 & 5.28996700 & 4.45862700 \\
\hline $\mathrm{C}$ & -4.97136200 & 3.46303300 & 3.30293600 \\
\hline $\mathrm{C}$ & -4.21902300 & 2.48061300 & 2.63372200 \\
\hline $\mathrm{H}$ & -4.73650400 & 1.65413800 & 2.14081300 \\
\hline $\mathrm{C}$ & -2.82702600 & 2.53158000 & 2.60833900 \\
\hline $\mathrm{H}$ & -2.23993200 & 1.75775500 & 2.11224400 \\
\hline $\mathrm{C}$ & -2.14678600 & 3.57901300 & 3.25947400 \\
\hline $\mathrm{C}$ & 1.34028700 & 2.89592700 & 3.06476500 \\
\hline $\mathrm{C}$ & 1.92831000 & 3.79579700 & 3.97533800 \\
\hline $\mathrm{H}$ & 1.28425800 & 4.49628100 & 4.50776200 \\
\hline $\mathrm{C}$ & 3.29842700 & 3.74569300 & 4.21035200 \\
\hline $\mathrm{H}$ & 3.73464300 & 4.41270900 & 4.96149500 \\
\hline $\mathrm{C}$ & 4.12980900 & 2.81294700 & 3.55467300 \\
\hline $\mathrm{C}$ & 3.53860000 & 1.93744400 & 2.63518900 \\
\hline $\mathrm{H}$ & 4.15621900 & 1.20127400 & 2.11649900 \\
\hline $\mathrm{C}$ & 2.16143600 & 1.97508800 & 2.39677800 \\
\hline $\mathrm{H}$ & 1.69058500 & 1.27622700 & 1.70237400 \\
\hline $\mathrm{C}$ & 5.60222000 & 2.76666300 & 3.93666500 \\
\hline $\mathrm{H}$ & 5.98041500 & 3.80433600 & 3.96832200 \\
\hline $\mathrm{H}$ & 5.66961600 & 2.40570400 & 4.97603200 \\
\hline $\mathrm{C}$ & 6.54497100 & 1.94881200 & 3.08573800 \\
\hline $\mathrm{C}$ & 7.28239800 & 0.89692400 & 3.63566300 \\
\hline $\mathrm{H}$ & 7.11560300 & 0.66036500 & 4.68586900 \\
\hline $\mathrm{C}$ & 8.23398500 & 0.18769000 & 2.88654200 \\
\hline $\mathrm{C}$ & 8.41592700 & 0.47913300 & 1.52213800 \\
\hline
\end{tabular}




\begin{tabular}{|c|c|c|c|}
\hline $\mathrm{C}$ & 7.68322200 & 1.54344600 & 0.97062400 \\
\hline $\mathrm{H}$ & 7.84378700 & 1.77996800 & -0.08240100 \\
\hline $\mathrm{C}$ & 6.78215000 & 2.28464800 & 1.74014700 \\
\hline $\mathrm{C}$ & 9.36888600 & -0.32307700 & 0.64923700 \\
\hline $\mathrm{H}$ & 9.93895100 & -1.01088700 & 1.28685200 \\
\hline $\mathrm{H}$ & 10.08836600 & 0.35888000 & 0.17575800 \\
\hline $\mathrm{C}$ & 8.66619300 & -1.12306600 & -0.43543300 \\
\hline $\mathrm{C}$ & 7.92065900 & -2.26516800 & -0.10889600 \\
\hline $\mathrm{H}$ & 7.86232400 & -2.59188500 & 0.93185400 \\
\hline $\mathrm{C}$ & 7.26370800 & -3.02161800 & -1.08106900 \\
\hline $\mathrm{C}$ & 7.25805200 & -2.61042900 & -2.42558900 \\
\hline $\mathrm{C}$ & 8.02050200 & -1.48408100 & -2.76051500 \\
\hline $\mathrm{H}$ & 8.03842800 & -1.16186100 & -3.80293700 \\
\hline $\mathrm{C}$ & 8.74880900 & -0.77157600 & -1.79717800 \\
\hline $\mathrm{C}$ & 8.70965700 & -1.29354600 & 4.72400400 \\
\hline $\mathrm{H}$ & 8.69436300 & -0.49370900 & 5.48490900 \\
\hline $\mathrm{H}$ & 7.70778800 & -1.75820600 & 4.70334200 \\
\hline $\mathrm{C}$ & 6.35528500 & 3.91748700 & 0.01513700 \\
\hline $\mathrm{H}$ & 7.41325900 & 4.23301800 & -0.05162100 \\
\hline $\mathrm{H}$ & 6.15964800 & 3.19902400 & -0.80159800 \\
\hline $\mathrm{C}$ & 7.28037100 & -5.38644200 & -0.94428300 \\
\hline $\mathrm{H}$ & 7.97625600 & -5.61053300 & -0.11367700 \\
\hline $\mathrm{H}$ & 7.88103400 & -5.33749400 & -1.86933900 \\
\hline $\mathrm{C}$ & 10.17961700 & 0.28461200 & -3.41023200 \\
\hline $\mathrm{H}$ & 10.45679600 & -0.74447700 & -3.69906000 \\
\hline $\mathrm{H}$ & 9.51127400 & 0.68555200 & -4.19355400 \\
\hline $\mathrm{C}$ & 6.47861200 & -3.36016400 & -3.48302300 \\
\hline $\mathrm{H}$ & 6.66811200 & -2.87661300 & -4.45609400 \\
\hline $\mathrm{H}$ & 6.88314300 & -4.37998000 & -3.59627200 \\
\hline $\mathrm{C}$ & 11.43658700 & 1.15399100 & -3.29083200 \\
\hline
\end{tabular}




\begin{tabular}{|c|c|c|c|}
\hline $\mathrm{H}$ & 11.16619900 & 2.19575900 & -3.07253200 \\
\hline $\mathrm{H}$ & 12.05797600 & 0.79000700 & -2.46039500 \\
\hline $\mathrm{C}$ & -11.43658700 & -1.15399100 & 3.29083200 \\
\hline $\mathrm{H}$ & -11.16619900 & -2.19575900 & 3.07253200 \\
\hline $\mathrm{H}$ & -12.05797600 & -0.79000700 & 2.46039500 \\
\hline $\mathrm{C}$ & 5.41744700 & 5.10383700 & -0.16359400 \\
\hline $\mathrm{H}$ & 4.37517000 & 4.76366200 & -0.09300900 \\
\hline $\mathrm{H}$ & 5.56223800 & 5.56346100 & -1.15023800 \\
\hline $\mathrm{C}$ & -5.41744700 & -5.10383700 & 0.16359400 \\
\hline $\mathrm{H}$ & -4.37517000 & -4.76366200 & 0.09300900 \\
\hline $\mathrm{H}$ & -5.56223800 & -5.56346100 & 1.15023800 \\
\hline $\mathrm{C}$ & -9.72885600 & 2.35024300 & -5.12096700 \\
\hline $\mathrm{H}$ & -9.30284800 & 2.98137900 & -5.91122000 \\
\hline $\mathrm{H}$ & -9.95872000 & 2.99539800 & -4.25971400 \\
\hline $\mathrm{C}$ & 9.72885600 & -2.35024300 & 5.12096700 \\
\hline $\mathrm{H}$ & 9.30284800 & -2.98137900 & 5.91122000 \\
\hline $\mathrm{H}$ & 9.95872000 & -2.99539800 & 4.25971400 \\
\hline $\mathrm{C}$ & 6.22010100 & -6.48319400 & -1.06694200 \\
\hline $\mathrm{H}$ & 5.65226100 & -6.57363000 & -0.13133200 \\
\hline $\mathrm{H}$ & 5.50869800 & -6.22259800 & -1.86356100 \\
\hline $\mathrm{C}$ & -6.22010100 & 6.48319400 & 1.06694200 \\
\hline $\mathrm{H}$ & -5.65226100 & 6.57363000 & 0.13133200 \\
\hline $\mathrm{H}$ & -5.50869800 & 6.22259800 & 1.86356100 \\
\hline $\mathrm{N}$ & 6.80930200 & -7.79093800 & -1.37992700 \\
\hline $\mathrm{C}$ & 7.02366600 & -8.79516300 & -0.51283900 \\
\hline $\mathrm{C}$ & 7.25762800 & -8.19144000 & -2.62776800 \\
\hline $\mathrm{H}$ & 6.76752900 & -8.78543000 & 0.54457800 \\
\hline $\mathrm{C}$ & 7.74492400 & -9.46176600 & -2.49235100 \\
\hline $\mathrm{H}$ & 7.18239700 & -7.55406400 & -3.50446300 \\
\hline $\mathrm{H}$ & 8.17831200 & -10.13935800 & -3.22505200 \\
\hline
\end{tabular}




\begin{tabular}{|c|c|c|c|}
\hline $\mathrm{N}$ & -6.80930200 & 7.79093800 & 1.37992700 \\
\hline $\mathrm{C}$ & -7.25762800 & 8.19144000 & 2.62776800 \\
\hline $\mathrm{C}$ & -7.02366600 & 8.79516300 & 0.51283900 \\
\hline $\mathrm{C}$ & -7.74492400 & 9.46176600 & 2.49235100 \\
\hline $\mathrm{H}$ & -7.18239700 & 7.55406400 & 3.50446300 \\
\hline $\mathrm{H}$ & -6.76752900 & 8.78543000 & -0.54457800 \\
\hline $\mathrm{H}$ & -8.17831200 & 10.13935800 & 3.22505200 \\
\hline $\mathrm{N}$ & 10.98444600 & -1.80633400 & 5.66228700 \\
\hline $\mathrm{C}$ & 11.55856200 & -2.18445600 & 6.86464600 \\
\hline $\mathrm{C}$ & 11.78314400 & -0.89841000 & 5.07532100 \\
\hline $\mathrm{C}$ & 12.72412400 & -1.48182100 & 6.99192500 \\
\hline $\mathrm{H}$ & 11.10091600 & -2.91621000 & 7.52609900 \\
\hline $\mathrm{H}$ & 11.58599900 & -0.41793700 & 4.12029500 \\
\hline $\mathrm{H}$ & 13.47020800 & -1.48285800 & 7.78408200 \\
\hline $\mathrm{N}$ & -10.98444600 & 1.80633400 & -5.66228700 \\
\hline $\mathrm{C}$ & -11.78314400 & 0.89841000 & -5.07532100 \\
\hline $\mathrm{C}$ & -11.55856200 & 2.18445600 & -6.86464600 \\
\hline $\mathrm{H}$ & -11.58599900 & 0.41793700 & -4.12029500 \\
\hline $\mathrm{C}$ & -12.72412400 & 1.48182100 & -6.99192500 \\
\hline $\mathrm{H}$ & -11.10091600 & 2.91621000 & -7.52609900 \\
\hline $\mathrm{H}$ & -13.47020800 & 1.48285800 & -7.78408200 \\
\hline $\mathrm{N}$ & -5.62121200 & -6.14356600 & -0.85063000 \\
\hline $\mathrm{C}$ & -5.10079300 & -6.12699500 & -2.08790400 \\
\hline $\mathrm{C}$ & -6.41731700 & -7.26925300 & -0.71824200 \\
\hline $\mathrm{H}$ & -4.43951800 & -5.35793200 & -2.48724700 \\
\hline $\mathrm{C}$ & -6.36117000 & -7.94012000 & -1.90749800 \\
\hline $\mathrm{H}$ & -6.93869700 & -7.51027100 & 0.20456800 \\
\hline $\mathrm{H}$ & -6.82558000 & -8.87293700 & -2.21988400 \\
\hline $\mathrm{N}$ & 5.62121200 & 6.14356600 & 0.85063000 \\
\hline $\mathrm{C}$ & 6.41731700 & 7.26925300 & 0.71824200 \\
\hline
\end{tabular}




\begin{tabular}{|c|c|c|c|}
\hline $\mathrm{C}$ & 5.10079300 & 6.12699500 & 2.08790400 \\
\hline $\mathrm{C}$ & 6.36117000 & 7.94012000 & 1.90749800 \\
\hline $\mathrm{H}$ & 6.93869700 & 7.51027100 & -0.20456800 \\
\hline $\mathrm{H}$ & 4.43951800 & 5.35793200 & 2.48724700 \\
\hline $\mathrm{H}$ & 6.82558000 & 8.87293700 & 2.21988400 \\
\hline $\mathrm{N}$ & 12.25294900 & 1.13999000 & -4.51020200 \\
\hline $\mathrm{C}$ & 12.39942200 & 2.15494900 & -5.38114500 \\
\hline $\mathrm{C}$ & 13.04379800 & 0.08438100 & -4.93731000 \\
\hline $\mathrm{H}$ & 11.91867000 & 3.12830800 & -5.30871400 \\
\hline $\mathrm{C}$ & 13.66738900 & 0.48802500 & -6.08468400 \\
\hline $\mathrm{H}$ & 13.11482900 & -0.85103100 & -4.38878600 \\
\hline $\mathrm{H}$ & 14.37612500 & -0.02938700 & -6.72816900 \\
\hline $\mathrm{N}$ & -12.25294900 & -1.13999000 & 4.51020200 \\
\hline $\mathrm{C}$ & -13.04379800 & -0.08438100 & 4.93731000 \\
\hline $\mathrm{C}$ & -12.39942200 & -2.15494900 & 5.38114500 \\
\hline $\mathrm{C}$ & -13.66738900 & -0.48802500 & 6.08468400 \\
\hline $\mathrm{H}$ & -13.11482900 & 0.85103100 & 4.38878600 \\
\hline $\mathrm{H}$ & -11.91867000 & -3.12830800 & 5.30871400 \\
\hline $\mathrm{H}$ & -14.37612500 & 0.02938700 & 6.72816900 \\
\hline $\mathrm{N}$ & 5.53452900 & 7.20982100 & 2.74362900 \\
\hline $\mathrm{N}$ & -5.53452900 & -7.20982100 & -2.74362900 \\
\hline $\mathrm{N}$ & 13.25070500 & 1.78105800 & -6.34061300 \\
\hline $\mathrm{N}$ & -13.25070500 & -1.78105800 & 6.34061300 \\
\hline $\mathrm{N}$ & -7.58814400 & 9.81667800 & 1.16565000 \\
\hline $\mathrm{N}$ & 7.58814400 & -9.81667800 & -1.16565000 \\
\hline $\mathrm{N}$ & 12.84189700 & -0.68771800 & 5.86696800 \\
\hline $\mathrm{N}$ & -12.84189700 & 0.68771800 & -5.86696800 \\
\hline $\mathrm{C}$ & -5.18209700 & -7.58185300 & -4.11806400 \\
\hline $\mathrm{H}$ & -6.09074700 & -7.62494100 & -4.73375700 \\
\hline $\mathrm{H}$ & -4.49528100 & -6.83046400 & -4.52442700 \\
\hline
\end{tabular}




\begin{tabular}{|c|c|c|c|}
\hline $\mathrm{H}$ & -4.69158400 & -8.56464000 & -4.11866000 \\
\hline $\mathrm{C}$ & -13.70118400 & -2.60687000 & 7.47254200 \\
\hline $\mathrm{H}$ & -13.20505100 & -3.58333300 & 7.42565800 \\
\hline $\mathrm{H}$ & -13.44203100 & -2.10593200 & 8.41488400 \\
\hline $\mathrm{H}$ & -14.78931200 & -2.74459300 & 7.41122200 \\
\hline $\mathrm{C}$ & -13.95849100 & -0.22760000 & -5.60011400 \\
\hline $\mathrm{H}$ & -14.89561800 & 0.34350400 & -5.54988900 \\
\hline $\mathrm{H}$ & -14.02361900 & -0.97060500 & -6.40650600 \\
\hline $\mathrm{H}$ & -13.78614100 & -0.73600800 & -4.64437200 \\
\hline $\mathrm{C}$ & -7.96708800 & 11.11430200 & 0.58579500 \\
\hline $\mathrm{H}$ & -9.04470600 & 11.27526200 & 0.72408500 \\
\hline $\mathrm{H}$ & -7.40537000 & 11.91517900 & 1.08538800 \\
\hline $\mathrm{H}$ & -7.73006700 & 11.11335000 & -0.48459300 \\
\hline $\mathrm{C}$ & 5.18209700 & 7.58185300 & 4.11806400 \\
\hline $\mathrm{H}$ & 4.49528100 & 6.83046400 & 4.52442700 \\
\hline $\mathrm{H}$ & 6.09074700 & 7.62494100 & 4.73375700 \\
\hline $\mathrm{H}$ & 4.69158400 & 8.56464000 & 4.11866000 \\
\hline $\mathrm{C}$ & 13.70118400 & 2.60687000 & -7.47254200 \\
\hline $\mathrm{H}$ & 13.44203100 & 2.10593200 & -8.41488400 \\
\hline $\mathrm{H}$ & 13.20505100 & 3.58333300 & -7.42565800 \\
\hline $\mathrm{H}$ & 14.78931200 & 2.74459300 & -7.41122200 \\
\hline $\mathrm{C}$ & 13.95849100 & 0.22760000 & 5.60011400 \\
\hline $\mathrm{H}$ & 14.02361900 & 0.97060500 & 6.40650600 \\
\hline $\mathrm{H}$ & 14.89561800 & -0.34350400 & 5.54988900 \\
\hline $\mathrm{H}$ & 13.78614100 & 0.73600800 & 4.64437200 \\
\hline $\mathrm{C}$ & 7.96708800 & -11.11430200 & -0.58579500 \\
\hline $\mathrm{H}$ & 7.40537000 & -11.91517900 & -1.08538800 \\
\hline $\mathrm{H}$ & 9.04470600 & -11.27526200 & -0.72408500 \\
\hline $\mathrm{H}$ & 7.73006700 & -11.11335000 & 0.48459300 \\
\hline
\end{tabular}




\section{$E, E-\mathbf{1} \bullet \mathrm{ANT}$}

81

\begin{tabular}{|c|c|c|c|}
\hline $\mathrm{O}$ & -9.32439600 & 2.74822200 & 2.78153600 \\
\hline $\mathrm{O}$ & -5.33996600 & 3.26529400 & -1.03453500 \\
\hline $\mathrm{O}$ & -7.58082400 & -3.45523500 & 1.62148700 \\
\hline $\mathrm{O}$ & -9.56244000 & 0.08149100 & -2.16049500 \\
\hline $\mathrm{N}$ & 1.25459900 & 4.71699800 & 1.22970200 \\
\hline $\mathrm{N}$ & 0.47409200 & 3.74338600 & 1.11473300 \\
\hline $\mathrm{C}$ & 3.46432800 & 5.51422600 & 1.61616400 \\
\hline $\mathrm{H}$ & 3.01557000 & 6.50777800 & 1.66934900 \\
\hline $\mathrm{C}$ & 4.82561900 & 5.32113300 & 1.84715300 \\
\hline $\mathrm{H}$ & 5.45315800 & 6.18136300 & 2.09854900 \\
\hline $\mathrm{C}$ & 5.38419100 & 4.03428700 & 1.81397100 \\
\hline $\mathrm{C}$ & 4.54620900 & 2.94784400 & 1.50803300 \\
\hline $\mathrm{H}$ & 4.96223300 & 1.94196900 & 1.46303300 \\
\hline $\mathrm{C}$ & 3.18493900 & 3.12654100 & 1.28113400 \\
\hline $\mathrm{H}$ & 2.53457000 & 2.27802500 & 1.06970900 \\
\hline $\mathrm{C}$ & 2.62736600 & 4.41769100 & 1.35164200 \\
\hline $\mathrm{C}$ & -0.90107100 & 4.08621700 & 1.09561000 \\
\hline $\mathrm{C}$ & -1.39211700 & 5.40179000 & 1.22243800 \\
\hline $\mathrm{H}$ & -0.67752500 & 6.22021200 & 1.31626700 \\
\hline $\mathrm{C}$ & -2.76372300 & 5.62754200 & 1.25728700 \\
\hline $\mathrm{H}$ & -3.13363700 & 6.65106300 & 1.37192900 \\
\hline $\mathrm{C}$ & -3.69363400 & 4.56824200 & 1.17334600 \\
\hline $\mathrm{C}$ & -3.19404800 & 3.26426300 & 1.06115600 \\
\hline $\mathrm{H}$ & -3.88155600 & 2.41835200 & 1.01449700 \\
\hline $\mathrm{C}$ & -1.81575300 & 3.02593400 & 1.01367500 \\
\hline $\mathrm{H}$ & -1.42599300 & 2.01106800 & 0.93512500 \\
\hline $\mathrm{C}$ & -5.16940100 & 4.93415000 & 1.19470700 \\
\hline $\mathrm{H}$ & -5.33154500 & 5.66426300 & 0.38055800 \\
\hline
\end{tabular}




\begin{tabular}{|c|c|c|c|}
\hline $\mathrm{H}$ & -5.36503600 & 5.49534800 & 2.12227800 \\
\hline $\mathrm{C}$ & -6.22273300 & 3.85949000 & 1.05843700 \\
\hline $\mathrm{C}$ & -7.22449300 & 3.72302400 & 2.02357700 \\
\hline $\mathrm{H}$ & -7.16934300 & 4.36424100 & 2.90288900 \\
\hline $\mathrm{C}$ & -8.29268400 & 2.82743900 & 1.86713000 \\
\hline $\mathrm{C}$ & -8.35365200 & 1.99338200 & 0.73517700 \\
\hline $\mathrm{C}$ & -7.35147100 & 2.13264200 & -0.23871300 \\
\hline $\mathrm{H}$ & -7.41420600 & 1.50988400 & -1.13124800 \\
\hline $\mathrm{C}$ & -6.31310200 & 3.05576200 & -0.09327100 \\
\hline $\mathrm{C}$ & -9.47393300 & 0.97717400 & 0.55439600 \\
\hline $\mathrm{H}$ & -10.01056200 & 0.87860300 & 1.50658800 \\
\hline $\mathrm{H}$ & -10.19503000 & 1.36223800 & -0.18342000 \\
\hline $\mathrm{C}$ & -8.98266500 & -0.38412200 & 0.10578000 \\
\hline $\mathrm{C}$ & -8.49799200 & -1.31008600 & 1.03843500 \\
\hline $\mathrm{H}$ & -8.52743900 & -1.06992400 & 2.10382400 \\
\hline $\mathrm{C}$ & -7.98252900 & -2.54914900 & 0.65073200 \\
\hline $\mathrm{C}$ & -7.85157300 & -2.87516600 & -0.70926600 \\
\hline $\mathrm{C}$ & -8.37708300 & -1.97324700 & -1.64475200 \\
\hline $\mathrm{H}$ & -8.30872000 & -2.22305000 & -2.70531700 \\
\hline $\mathrm{C}$ & -8.97779500 & -0.77158800 & -1.24948500 \\
\hline $\mathrm{C}$ & -9.07966500 & 3.17830200 & 4.11145600 \\
\hline $\mathrm{H}$ & -8.86217700 & 4.25956700 & 4.15698200 \\
\hline $\mathrm{H}$ & -8.20788800 & 2.64173500 & 4.52555900 \\
\hline $\mathrm{C}$ & -5.15692200 & 2.37055100 & -2.10087700 \\
\hline $\mathrm{H}$ & -5.92202300 & 2.52695900 & -2.88454400 \\
\hline $\mathrm{H}$ & -5.22096600 & 1.31936700 & -1.76802200 \\
\hline $\mathrm{C}$ & -8.58391200 & -4.37150900 & 2.02661400 \\
\hline $\mathrm{H}$ & -9.39798100 & -3.85524000 & 2.56841600 \\
\hline $\mathrm{H}$ & -9.03607400 & -4.87151000 & 1.15040400 \\
\hline $\mathrm{C}$ & -10.22088400 & -0.47552900 & -3.28327500 \\
\hline
\end{tabular}




\begin{tabular}{|c|c|c|c|}
\hline $\mathrm{H}$ & -10.63092700 & -1.47007000 & -3.03626000 \\
\hline $\mathrm{H}$ & -9.53100000 & -0.59830600 & -4.13752100 \\
\hline $\mathrm{C}$ & -7.13230200 & -4.12960400 & -1.16385500 \\
\hline $\mathrm{H}$ & -7.57706700 & -4.47917200 & -2.10621500 \\
\hline $\mathrm{H}$ & -7.28486400 & -4.93031600 & -0.42567600 \\
\hline $\mathrm{O}$ & 8.67646600 & -1.17707100 & -2.90693000 \\
\hline $\mathrm{O}$ & 5.72238800 & -4.37042100 & 0.52678800 \\
\hline $\mathrm{O}$ & 7.32625800 & 3.53806400 & -0.62182600 \\
\hline $\mathrm{O}$ & 9.18472900 & -0.51321300 & 2.67768400 \\
\hline $\mathrm{N}$ & -1.46543000 & -3.78259100 & -2.04687400 \\
\hline $\mathrm{N}$ & -0.67421500 & -3.70013700 & -1.07907900 \\
\hline $\mathrm{C}$ & -3.70519700 & -3.88913300 & -2.84407600 \\
\hline $\mathrm{H}$ & -3.26599700 & -3.94439200 & -3.84185300 \\
\hline $\mathrm{C}$ & -5.08544400 & -3.97368100 & -2.65479400 \\
\hline $\mathrm{H}$ & -5.73931300 & -4.10224600 & -3.52101700 \\
\hline $\mathrm{C}$ & -5.63461800 & -3.94501700 & -1.36588900 \\
\hline $\mathrm{C}$ & -4.76476500 & -3.79135900 & -0.26604600 \\
\hline $\mathrm{H}$ & -5.18502900 & -3.75812300 & 0.74210300 \\
\hline $\mathrm{C}$ & -3.38760600 & -3.71210000 & -0.44498700 \\
\hline $\mathrm{H}$ & -2.70827500 & -3.62090000 & 0.40209600 \\
\hline $\mathrm{C}$ & -2.84336400 & -3.77428800 & -1.74356100 \\
\hline $\mathrm{C}$ & 0.69624200 & -3.81105900 & -1.42052900 \\
\hline $\mathrm{C}$ & 1.16350500 & -4.24197600 & -2.67610800 \\
\hline $\mathrm{H}$ & 0.43706700 & -4.47871800 & -3.45405900 \\
\hline $\mathrm{C}$ & 2.53136500 & -4.37376600 & -2.89973100 \\
\hline $\mathrm{H}$ & 2.87723200 & -4.72644400 & -3.87567500 \\
\hline $\mathrm{C}$ & 3.47833400 & -4.06931100 & -1.90044800 \\
\hline $\mathrm{C}$ & 3.00084200 & -3.60630900 & -0.66259100 \\
\hline $\mathrm{H}$ & 3.70097800 & -3.32290200 & 0.12154400 \\
\hline $\mathrm{C}$ & 1.62857700 & -3.50148400 & -0.42088400 \\
\hline
\end{tabular}




\begin{tabular}{|c|c|c|c|}
\hline $\mathrm{H}$ & 1.25489800 & -3.15444200 & 0.54273900 \\
\hline $\mathrm{C}$ & 4.94492400 & -4.37065400 & -2.18483000 \\
\hline $\mathrm{H}$ & 5.15419900 & -5.38909800 & -1.80773100 \\
\hline $\mathrm{H}$ & 5.07078800 & -4.43562000 & -3.27480100 \\
\hline $\mathrm{C}$ & 6.03091900 & -3.46791000 & -1.63787000 \\
\hline $\mathrm{C}$ & 6.76852300 & -2.64835300 & -2.50345200 \\
\hline $\mathrm{H}$ & 6.48404700 & -2.64274000 & -3.55501000 \\
\hline $\mathrm{C}$ & 7.86490800 & -1.89941800 & -2.05717500 \\
\hline $\mathrm{C}$ & 8.20071200 & -1.88364700 & -0.68837500 \\
\hline $\mathrm{C}$ & 7.47181700 & -2.71293800 & 0.17307600 \\
\hline $\mathrm{H}$ & 7.73690700 & -2.71457400 & 1.23191900 \\
\hline $\mathrm{C}$ & 6.42594000 & -3.51719600 & -0.29205300 \\
\hline $\mathrm{C}$ & 9.28607500 & -0.97111100 & -0.14161600 \\
\hline $\mathrm{H}$ & 9.97170500 & -0.69478700 & -0.95317300 \\
\hline $\mathrm{H}$ & 9.86655200 & -1.51092100 & 0.61721800 \\
\hline $\mathrm{C}$ & 8.72126200 & 0.29649300 & 0.47935400 \\
\hline $\mathrm{C}$ & 8.25857800 & 1.34621000 & -0.32766900 \\
\hline $\mathrm{H}$ & 8.33749700 & 1.26517500 & -1.41376300 \\
\hline $\mathrm{C}$ & 7.71705200 & 2.51191800 & 0.21701400 \\
\hline $\mathrm{C}$ & 7.53654300 & 2.63564700 & 1.60598600 \\
\hline $\mathrm{C}$ & 8.01912600 & 1.60062700 & 2.41657100 \\
\hline $\mathrm{H}$ & 7.89859400 & 1.68890800 & 3.49760600 \\
\hline $\mathrm{C}$ & 8.64110700 & 0.46686800 & 1.87527600 \\
\hline $\mathrm{C}$ & 8.33997500 & -1.10148800 & -4.28002900 \\
\hline $\mathrm{H}$ & 8.19138500 & -2.10546500 & -4.71438400 \\
\hline $\mathrm{H}$ & 7.40164500 & -0.53358700 & -4.41200800 \\
\hline $\mathrm{C}$ & 6.38587700 & -5.04750400 & 1.57286200 \\
\hline $\mathrm{H}$ & 7.30467500 & -5.53351100 & 1.19801900 \\
\hline $\mathrm{H}$ & 6.66765600 & -4.36442400 & 2.39347400 \\
\hline $\mathrm{C}$ & 8.21218400 & 4.63874800 & -0.70961600 \\
\hline
\end{tabular}




\begin{tabular}{|c|c|c|c|}
\hline $\mathrm{H}$ & 9.00243300 & 4.44990900 & -1.46054700 \\
\hline $\mathrm{H}$ & 8.71627000 & 4.82532000 & 0.25426400 \\
\hline $\mathrm{C}$ & 9.74457700 & -0.13182900 & 3.92190400 \\
\hline $\mathrm{H}$ & 10.18408900 & 0.87847100 & 3.85381900 \\
\hline $\mathrm{H}$ & 8.98146700 & -0.11432100 & 4.72048300 \\
\hline $\mathrm{C}$ & 6.83877200 & 3.83384700 & 2.20958200 \\
\hline $\mathrm{H}$ & 6.88374200 & 3.74319500 & 3.30785800 \\
\hline $\mathrm{H}$ & 7.39563600 & 4.75731300 & 1.98159500 \\
\hline $\mathrm{C}$ & 10.83632100 & -1.15680800 & 4.25192700 \\
\hline $\mathrm{H}$ & 10.39979100 & -2.15379300 & 4.39868200 \\
\hline $\mathrm{H}$ & 11.54330900 & -1.22266900 & 3.41281900 \\
\hline $\mathrm{C}$ & -11.35660700 & 0.48735900 & -3.65071800 \\
\hline $\mathrm{H}$ & -10.95127900 & 1.46238700 & -3.95243300 \\
\hline $\mathrm{H}$ & -11.99696100 & 0.65000000 & -2.77212200 \\
\hline $\mathrm{C}$ & 5.41064800 & -6.07060900 & 2.14088400 \\
\hline $\mathrm{H}$ & 4.51537100 & -5.56023100 & 2.52234700 \\
\hline $\mathrm{H}$ & 5.87226800 & -6.61293600 & 2.97672800 \\
\hline $\mathrm{C}$ & -3.75228200 & 2.60651100 & -2.64697200 \\
\hline $\mathrm{H}$ & -3.00534600 & 2.32520200 & -1.89280800 \\
\hline $\mathrm{H}$ & -3.58078300 & 1.99466400 & -3.54331900 \\
\hline $\mathrm{C}$ & -10.29482200 & 2.87216400 & 4.97309800 \\
\hline $\mathrm{H}$ & -9.99241000 & 2.86482400 & 6.02809800 \\
\hline $\mathrm{H}$ & -10.69113400 & 1.87530600 & 4.72788600 \\
\hline $\mathrm{C}$ & 9.45418400 & -0.38239400 & -5.02555000 \\
\hline $\mathrm{H}$ & 9.06727400 & -0.01153100 & -5.98315300 \\
\hline $\mathrm{H}$ & 9.79939500 & 0.48478200 & -4.44312900 \\
\hline $\mathrm{C}$ & 7.37350800 & 5.85678000 & -1.10540900 \\
\hline $\mathrm{H}$ & 6.92251000 & 5.70789600 & -2.09555700 \\
\hline $\mathrm{H}$ & 6.55403800 & 5.98948000 & -0.38485300 \\
\hline $\mathrm{C}$ & -7.90546100 & -5.40152000 & 2.93171700 \\
\hline
\end{tabular}




\begin{tabular}{|c|c|c|c|}
\hline $\mathrm{H}$ & -7.50868200 & -4.91590800 & 3.83322100 \\
\hline $\mathrm{H}$ & -7.05873000 & -5.86142200 & 2.40208600 \\
\hline $\mathrm{N}$ & 8.16618100 & 7.09202300 & -1.13881500 \\
\hline $\mathrm{C}$ & 8.64220300 & 7.70406300 & -2.23692900 \\
\hline $\mathrm{C}$ & 8.55962500 & 7.82277500 & -0.02971000 \\
\hline $\mathrm{H}$ & 8.48973800 & 7.37532000 & -3.26282100 \\
\hline $\mathrm{C}$ & 9.28190200 & 8.89031400 & -0.48589400 \\
\hline $\mathrm{H}$ & 8.28557700 & 7.53681100 & 0.98204000 \\
\hline $\mathrm{H}$ & 9.76011700 & 9.70716900 & 0.05074200 \\
\hline $\mathrm{N}$ & -8.81994100 & -6.47061400 & 3.34899100 \\
\hline $\mathrm{C}$ & -9.28029900 & -7.49839700 & 2.54141900 \\
\hline $\mathrm{C}$ & -9.33848300 & -6.63600300 & 4.57862800 \\
\hline $\mathrm{C}$ & -10.08784100 & -8.28566100 & 3.31406900 \\
\hline $\mathrm{H}$ & -8.98787900 & -7.59726100 & 1.49951200 \\
\hline $\mathrm{H}$ & -9.15006400 & -5.99575500 & 5.43803500 \\
\hline $\mathrm{H}$ & -10.63684200 & -9.19358500 & 3.07281900 \\
\hline $\mathrm{N}$ & 10.61095700 & -1.23145000 & -5.35025800 \\
\hline $\mathrm{C}$ & 11.10933700 & -1.43950300 & -6.62595100 \\
\hline $\mathrm{C}$ & 11.38285400 & -1.90183100 & -4.47711100 \\
\hline $\mathrm{C}$ & 12.20140400 & -2.25235700 & -6.50332700 \\
\hline $\mathrm{H}$ & 10.65830200 & -0.99401800 & -7.50941900 \\
\hline $\mathrm{H}$ & 11.23286100 & -1.92869800 & -3.40093000 \\
\hline $\mathrm{H}$ & 12.87741200 & -2.65100000 & -7.25724900 \\
\hline $\mathrm{N}$ & -11.37913000 & 3.86095600 & 4.86414600 \\
\hline $\mathrm{C}$ & -11.94660000 & 4.30542500 & 3.72982500 \\
\hline $\mathrm{C}$ & -12.01196400 & 4.46434300 & 5.93817300 \\
\hline $\mathrm{H}$ & -11.65203900 & 4.00682300 & 2.72677300 \\
\hline $\mathrm{C}$ & -12.97571200 & 5.28716000 & 5.42581100 \\
\hline $\mathrm{H}$ & -11.73264000 & 4.26261800 & 6.96958800 \\
\hline $\mathrm{H}$ & -13.69046700 & 5.93880300 & 5.92441000 \\
\hline
\end{tabular}




\begin{tabular}{|c|c|c|c|}
\hline $\mathrm{N}$ & -3.52602500 & 4.01125000 & -2.99571000 \\
\hline $\mathrm{C}$ & -2.99540200 & 4.92266300 & -2.16823800 \\
\hline $\mathrm{C}$ & -3.92162200 & 4.64496900 & -4.16201900 \\
\hline $\mathrm{H}$ & -2.61821000 & 4.72626200 & -1.16710100 \\
\hline $\mathrm{C}$ & -3.60798400 & 5.96773000 & -4.02266600 \\
\hline $\mathrm{H}$ & -4.37517900 & 4.10981800 & -4.99189400 \\
\hline $\mathrm{H}$ & -3.73554500 & 6.80487600 & -4.70509500 \\
\hline $\mathrm{N}$ & 4.97592900 & -7.05910500 & 1.14860500 \\
\hline $\mathrm{C}$ & 5.49819300 & -8.33035400 & 0.97158600 \\
\hline $\mathrm{C}$ & 4.02615000 & -6.85080100 & 0.22339400 \\
\hline $\mathrm{C}$ & 4.83595100 & -8.88949400 & -0.08547500 \\
\hline $\mathrm{H}$ & 6.27706000 & -8.73685900 & 1.61217900 \\
\hline $\mathrm{H}$ & 3.44874700 & -5.93509100 & 0.09857900 \\
\hline $\mathrm{H}$ & 4.92924400 & -9.87285500 & -0.54109600 \\
\hline $\mathrm{N}$ & 11.59478800 & -0.80904200 & 5.45907400 \\
\hline $\mathrm{C}$ & 11.53078700 & -1.43672000 & 6.64755900 \\
\hline $\mathrm{C}$ & 12.54136200 & 0.19982200 & 5.55190600 \\
\hline $\mathrm{H}$ & 10.89100400 & -2.28428400 & 6.88444100 \\
\hline $\mathrm{C}$ & 13.04310000 & 0.16438200 & 6.82279200 \\
\hline $\mathrm{H}$ & 12.79444600 & 0.84214400 & 4.71265400 \\
\hline $\mathrm{H}$ & 13.80756300 & 0.77265800 & 7.30215000 \\
\hline $\mathrm{N}$ & -12.19894200 & -0.01449600 & -4.74276700 \\
\hline $\mathrm{C}$ & -13.09632600 & -1.06729800 & -4.65004700 \\
\hline $\mathrm{C}$ & -12.27631200 & 0.49616500 & -5.98506300 \\
\hline $\mathrm{C}$ & -13.71229100 & -1.17853500 & -5.86522500 \\
\hline $\mathrm{H}$ & -13.23829600 & -1.63526100 & -3.73455100 \\
\hline $\mathrm{H}$ & -11.70714500 & 1.34461400 & -6.35934500 \\
\hline $\mathrm{H}$ & -14.48320600 & -1.86430900 & -6.21098900 \\
\hline $\mathrm{N}$ & 3.92573300 & -7.94765900 & -0.53502700 \\
\hline $\mathrm{N}$ & -3.03205200 & 6.11576500 & -2.77211200 \\
\hline
\end{tabular}




\begin{tabular}{|c|c|c|c|}
\hline $\mathrm{N}$ & 12.39883900 & -0.86344700 & 7.48581500 \\
\hline $\mathrm{N}$ & -13.18547200 & -0.19374400 & -6.67983000 \\
\hline $\mathrm{N}$ & -10.10808900 & -7.72872500 & 4.57916000 \\
\hline $\mathrm{N}$ & 9.31947800 & 8.79510100 & -1.86435600 \\
\hline $\mathrm{N}$ & 12.35145900 & -2.52692900 & -5.15721200 \\
\hline $\mathrm{N}$ & -12.91515600 & 5.17241300 & 4.04982400 \\
\hline $\mathrm{C}$ & -2.53567500 & 7.37457000 & -2.20773100 \\
\hline $\mathrm{H}$ & -3.35629200 & 8.10279500 & -2.15474500 \\
\hline $\mathrm{H}$ & -2.14962400 & 7.17944300 & -1.20061300 \\
\hline $\mathrm{H}$ & -1.73257100 & 7.77264400 & -2.84248300 \\
\hline $\mathrm{C}$ & -13.58988800 & 0.05923700 & -8.07220800 \\
\hline $\mathrm{H}$ & -13.00061100 & 0.89258600 & -8.47231800 \\
\hline $\mathrm{H}$ & -13.41028400 & -0.84219000 & -8.67324500 \\
\hline $\mathrm{H}$ & -14.65716200 & 0.31806300 & -8.09930100 \\
\hline $\mathrm{C}$ & -13.78256400 & 5.89286400 & 3.10976000 \\
\hline $\mathrm{H}$ & -14.83067800 & 5.62443400 & 3.30006800 \\
\hline $\mathrm{H}$ & -13.64987700 & 6.97495300 & 3.24450700 \\
\hline $\mathrm{H}$ & -13.51179800 & 5.61406000 & 2.08477800 \\
\hline $\mathrm{C}$ & -10.84212400 & -8.27303400 & 5.73230500 \\
\hline $\mathrm{H}$ & -11.91537600 & -8.30280400 & 5.50098600 \\
\hline $\mathrm{H}$ & -10.48030300 & -9.28754300 & 5.94774800 \\
\hline $\mathrm{H}$ & -10.67087700 & -7.63055200 & 6.60395100 \\
\hline $\mathrm{C}$ & 2.98580700 & -8.12994400 & -1.64570200 \\
\hline $\mathrm{H}$ & 2.50181700 & -7.16947900 & -1.86303300 \\
\hline $\mathrm{H}$ & 3.53410900 & -8.47353500 & -2.53282300 \\
\hline $\mathrm{H}$ & 2.22833800 & -8.87729100 & -1.37184600 \\
\hline $\mathrm{C}$ & 12.65804900 & -1.26593400 & 8.87768000 \\
\hline $\mathrm{H}$ & 12.45296000 & -0.41904000 & 9.54613300 \\
\hline $\mathrm{H}$ & 12.00447700 & -2.10706400 & 9.13688000 \\
\hline $\mathrm{H}$ & 13.70815900 & -1.57274000 & 8.97705200 \\
\hline
\end{tabular}




\begin{tabular}{|c|c|c|c|}
\hline $\mathrm{C}$ & 13.41695700 & -3.36047700 & -4.58528000 \\
\hline $\mathrm{H}$ & 13.36838600 & -4.36710600 & -5.02206400 \\
\hline $\mathrm{H}$ & 14.39359600 & -2.90962000 & -4.80874700 \\
\hline $\mathrm{H}$ & 13.28094400 & -3.42302600 & -3.49937100 \\
\hline $\mathrm{C}$ & 9.98107500 & 9.75522700 & -2.76161400 \\
\hline $\mathrm{H}$ & 9.52066300 & 10.74510000 & -2.63991700 \\
\hline $\mathrm{H}$ & 11.04950600 & 9.80980700 & -2.51327100 \\
\hline $\mathrm{H}$ & 9.86162600 & 9.42027500 & -3.79864900 \\
\hline $\mathrm{C}$ & 4.65305200 & -0.56356700 & 1.04872900 \\
\hline $\mathrm{C}$ & 3.40662300 & -0.58907300 & 1.62450900 \\
\hline $\mathrm{C}$ & 2.22552400 & -0.45064100 & 0.82660600 \\
\hline $\mathrm{C}$ & 2.37151400 & -0.28014500 & -0.60128100 \\
\hline $\mathrm{C}$ & 3.68995300 & -0.23393800 & -1.15469400 \\
\hline $\mathrm{C}$ & 4.79712700 & -0.37439000 & -0.35769200 \\
\hline $\mathrm{C}$ & 0.93758400 & -0.51148000 & 1.37964900 \\
\hline $\mathrm{C}$ & 1.22234400 & -0.20633900 & -1.40068300 \\
\hline $\mathrm{C}$ & -0.06392400 & -0.30690200 & -0.85299100 \\
\hline $\mathrm{C}$ & -0.21080600 & -0.45898400 & 0.57705400 \\
\hline $\mathrm{C}$ & -1.52974800 & -0.57361300 & 1.12328200 \\
\hline $\mathrm{H}$ & -1.63820900 & -0.68215700 & 2.20511900 \\
\hline $\mathrm{C}$ & -2.63528600 & -0.55809800 & 0.31050800 \\
\hline $\mathrm{C}$ & -2.48815000 & -0.42853700 & -1.10292500 \\
\hline $\mathrm{C}$ & -1.24276200 & -0.30020800 & -1.66545600 \\
\hline $\mathrm{H}$ & 0.82651900 & -0.62916300 & 2.46072100 \\
\hline $\mathrm{H}$ & 5.54909000 & -0.67394600 & 1.66362300 \\
\hline $\mathrm{H}$ & 3.29409000 & -0.71662000 & 2.70404600 \\
\hline $\mathrm{H}$ & 3.79681900 & -0.10325200 & -2.23419100 \\
\hline $\mathrm{H}$ & 5.79738100 & -0.34870800 & -0.79033000 \\
\hline $\mathrm{H}$ & 1.33378600 & -0.09917700 & -2.48279500 \\
\hline $\mathrm{H}$ & -3.63427300 & -0.66873700 & 0.73761800 \\
\hline
\end{tabular}




$\begin{array}{llll}\mathrm{H} & -3.37551500 & -0.47582200 & -1.73887400 \\ \mathrm{H} & -1.12653000 & -0.22613400 & -2.74969200\end{array}$

\section{E,E-1•PHE}

81

O

O

O

O

N

$\mathrm{N}$

C

H

C

H

C

C

$\mathrm{H}$

C

H

C

C

C

H

C

H

C

C

H

C $\begin{array}{lll}9.16243800 & -2.29236400 \quad 3.05284000\end{array}$

$\begin{array}{lll}5.35079600 & -3.64061700 & -0.73178400\end{array}$

$\begin{array}{lll}7.31071800 & 3.57053400 & 1.03365900\end{array}$

$\begin{array}{lll}9.55734500 & -0.31233700 & -2.22139500\end{array}$

$\begin{array}{lll}-1.28662700 & -4.85893500 & 1.38301400\end{array}$

$\begin{array}{lll}-0.50814000 & -3.87868000 & 1.33041800\end{array}$

$\begin{array}{lll}-3.53683600 & -5.65845600 & 1.42596500\end{array}$

$\begin{array}{lll}-3.10784300 & -6.65892500 & 1.34533200\end{array}$

$\begin{array}{lll}-4.91383600 & -5.46812800 & 1.54301200\end{array}$

$\begin{array}{lll}-5.57428500 & -6.34002500 & 1.56471100\end{array}$

$\begin{array}{lll}-5.45154500 & -4.18031900 & 1.68760100\end{array}$

$\begin{array}{lll}-4.57034300 & -3.08572500 & 1.69378500\end{array}$

$\begin{array}{lll}-4.96874100 & -2.07879700 & 1.81141700\end{array}$

$\begin{array}{lll}-3.19670100 & -3.26016800 & 1.56641500\end{array}$

$\begin{array}{lll}-2.51661800 & -2.40894700 & 1.58424600\end{array}$

$\begin{array}{lll}-2.66492300 & -4.55788200 & 1.43863600\end{array}$

$\begin{array}{lll}0.86970800 & -4.19593700 & 1.39308600\end{array}$

$\begin{array}{lll}1.38307000 & -5.47181500 & 1.70094300\end{array}$

$\begin{array}{lll}0.68549700 & -6.29085000 & 1.87991400\end{array}$

$\begin{array}{lll}2.75892500 & -5.65399300 & 1.80672700\end{array}$

$\begin{array}{lll}3.14703200 & -6.64128300 & 2.07679700\end{array}$

$\begin{array}{lll}3.66788100 & -4.59092800 & 1.60894700\end{array}$

$\begin{array}{lll}3.14360500 & -3.32759000 & 1.30698200\end{array}$

$\begin{array}{lll}3.81444500 & -2.47902300 & 1.16331200\end{array}$

$\begin{array}{lll}1.76336800 & -3.13317700 & 1.20164000\end{array}$ 


\begin{tabular}{|c|c|c|c|}
\hline $\mathrm{H}$ & 1.35011600 & -2.14984500 & 0.98433200 \\
\hline $\mathrm{C}$ & 5.15259400 & -4.89661300 & 1.73970100 \\
\hline $\mathrm{H}$ & 5.37152300 & -5.74738900 & 1.06844000 \\
\hline $\mathrm{H}$ & 5.33074800 & -5.28348800 & 2.75575700 \\
\hline $\mathrm{C}$ & 6.17141000 & -3.81599100 & 1.45627200 \\
\hline $\mathrm{C}$ & 7.12746200 & -3.46936200 & 2.41443100 \\
\hline $\mathrm{H}$ & 7.06532800 & -3.95384100 & 3.38845300 \\
\hline $\mathrm{C}$ & 8.16443100 & -2.56503800 & 2.13864800 \\
\hline $\mathrm{C}$ & 8.23201900 & -1.92860900 & 0.88464200 \\
\hline $\mathrm{C}$ & 7.27443700 & -2.27801200 & -0.08212600 \\
\hline $\mathrm{H}$ & 7.34539300 & -1.80979500 & -1.06467200 \\
\hline $\mathrm{C}$ & 6.27499900 & -3.21718000 & 0.18661000 \\
\hline $\mathrm{C}$ & 9.32338400 & -0.91550300 & 0.56636500 \\
\hline $\mathrm{H}$ & 9.85620900 & -0.68029500 & 1.49650300 \\
\hline $\mathrm{H}$ & 10.05432600 & -1.37831000 & -0.11431400 \\
\hline $\mathrm{C}$ & 8.81979300 & 0.37210900 & -0.05824300 \\
\hline $\mathrm{C}$ & 8.26201200 & 1.38062100 & 0.74170200 \\
\hline $\mathrm{H}$ & 8.20905000 & 1.24442000 & 1.82432900 \\
\hline $\mathrm{C}$ & 7.79192700 & 2.57809700 & 0.19994100 \\
\hline $\mathrm{C}$ & 7.78265200 & 2.78017200 & -1.19095200 \\
\hline $\mathrm{C}$ & 8.36323100 & 1.78941600 & -1.99302500 \\
\hline $\mathrm{H}$ & 8.38149400 & 1.94075000 & -3.07370600 \\
\hline $\mathrm{C}$ & 8.91571300 & 0.62461900 & -1.44108400 \\
\hline $\mathrm{C}$ & 8.90388200 & -2.53138300 & 4.42727500 \\
\hline $\mathrm{H}$ & 8.72965700 & -3.60271300 & 4.62872500 \\
\hline $\mathrm{H}$ & 8.00118900 & -1.97864600 & 4.74197600 \\
\hline $\mathrm{C}$ & 5.20773700 & -3.00446700 & -1.97367000 \\
\hline $\mathrm{H}$ & 6.04317100 & -3.26144900 & -2.65173800 \\
\hline $\mathrm{H}$ & 5.18022200 & -1.90470100 & -1.86878100 \\
\hline $\mathrm{C}$ & 8.24015400 & 4.58369800 & 1.37108300 \\
\hline
\end{tabular}




\begin{tabular}{|c|c|c|c|}
\hline $\mathrm{H}$ & 8.91220800 & 4.25205400 & 2.18481500 \\
\hline $\mathrm{H}$ & 8.87591600 & 4.84504500 & 0.50638100 \\
\hline $\mathrm{C}$ & 10.25757700 & 0.12908300 & -3.36990300 \\
\hline $\mathrm{H}$ & 10.68910200 & 1.13059500 & -3.19796400 \\
\hline $\mathrm{H}$ & 9.59278400 & 0.19545400 & -4.24997300 \\
\hline $\mathrm{C}$ & 7.16998300 & 4.01917100 & -1.80130600 \\
\hline $\mathrm{H}$ & 7.40038900 & 4.02652400 & -2.87974900 \\
\hline $\mathrm{H}$ & 7.66391700 & 4.92065800 & -1.40229700 \\
\hline $\mathrm{O}$ & -8.87402600 & 1.71316000 & -3.01459300 \\
\hline $\mathrm{O}$ & -5.27461200 & 4.03364000 & 0.49953600 \\
\hline $\mathrm{O}$ & -7.10884100 & -3.44416000 & -0.89947900 \\
\hline $\mathrm{O}$ & -9.28279000 & 0.34700500 & 2.51385100 \\
\hline $\mathrm{N}$ & 1.48195300 & 4.84239600 & -1.67928800 \\
\hline $\mathrm{N}$ & 0.71479200 & 3.91025500 & -1.34415200 \\
\hline $\mathrm{C}$ & 3.72440700 & 5.64786200 & -1.87225400 \\
\hline $\mathrm{H}$ & 3.28457500 & 6.62255300 & -2.09137600 \\
\hline $\mathrm{C}$ & 5.10496200 & 5.45916000 & -1.88414100 \\
\hline $\mathrm{H}$ & 5.75762200 & 6.30383000 & -2.12237900 \\
\hline $\mathrm{C}$ & 5.66515400 & 4.19379500 & -1.64369200 \\
\hline $\mathrm{C}$ & 4.79797800 & 3.12477300 & -1.35523900 \\
\hline $\mathrm{H}$ & 5.21540800 & 2.13366100 & -1.16137500 \\
\hline $\mathrm{C}$ & 3.41697500 & 3.30452700 & -1.32974700 \\
\hline $\mathrm{H}$ & 2.73904000 & 2.47594600 & -1.12760600 \\
\hline $\mathrm{C}$ & 2.86564500 & 4.57102300 & -1.60011100 \\
\hline $\mathrm{C}$ & -0.66478100 & 4.16095300 & -1.53888400 \\
\hline $\mathrm{C}$ & -1.18581500 & 5.26555200 & -2.24240000 \\
\hline $\mathrm{H}$ & -0.49513400 & 6.00626600 & -2.64738700 \\
\hline $\mathrm{C}$ & -2.55963500 & 5.37085600 & -2.43624100 \\
\hline $\mathrm{H}$ & -2.95370100 & 6.21810200 & -3.00604000 \\
\hline $\mathrm{C}$ & -3.45862500 & 4.39809100 & -1.94466100 \\
\hline
\end{tabular}




\begin{tabular}{|c|c|c|c|}
\hline $\mathrm{C}$ & -2.92931100 & 3.30164400 & -1.25153300 \\
\hline $\mathrm{H}$ & -3.58820500 & 2.52098200 & -0.87082300 \\
\hline $\mathrm{C}$ & -1.54965900 & 3.18986300 & -1.05065100 \\
\hline $\mathrm{H}$ & -1.12579500 & 2.33591100 & -0.52825400 \\
\hline $\mathrm{C}$ & -4.94127200 & 4.62431100 & -2.20056200 \\
\hline $\mathrm{H}$ & -5.19992100 & 5.60726600 & -1.76491900 \\
\hline $\mathrm{H}$ & -5.08056200 & 4.75021200 & -3.28593100 \\
\hline $\mathrm{C}$ & -5.96526300 & 3.62897600 & -1.70336600 \\
\hline $\mathrm{C}$ & -6.86259500 & 3.03277900 & -2.59510700 \\
\hline $\mathrm{H}$ & -6.73512300 & 3.24933500 & -3.65506900 \\
\hline $\mathrm{C}$ & -7.92100900 & 2.22342900 & -2.15553300 \\
\hline $\mathrm{C}$ & -8.06574800 & 1.93774700 & -0.78561800 \\
\hline $\mathrm{C}$ & -7.17286200 & 2.54352500 & 0.11216500 \\
\hline $\mathrm{H}$ & -7.30887800 & 2.34390600 & 1.17601400 \\
\hline $\mathrm{C}$ & -6.14956200 & 3.38617200 & -0.32962700 \\
\hline $\mathrm{C}$ & -9.15047100 & 1.00791100 & -0.26586100 \\
\hline $\mathrm{H}$ & -9.83892700 & 0.77051700 & -1.08732600 \\
\hline $\mathrm{H}$ & -9.73071900 & 1.52657300 & 0.50905600 \\
\hline $\mathrm{C}$ & -8.61279800 & -0.29035900 & 0.31484300 \\
\hline $\mathrm{C}$ & -8.06754100 & -1.27499100 & -0.52255600 \\
\hline $\mathrm{H}$ & -8.04116800 & -1.10798200 & -1.60184700 \\
\hline $\mathrm{C}$ & -7.58141600 & -2.48476800 & -0.02344800 \\
\hline $\mathrm{C}$ & -7.54985200 & -2.72790400 & 1.36134800 \\
\hline $\mathrm{C}$ & -8.10700500 & -1.75499700 & 2.20092500 \\
\hline $\mathrm{H}$ & -8.09983000 & -1.93511100 & 3.27711900 \\
\hline $\mathrm{C}$ & -8.66777900 & -0.57287400 & 1.69439500 \\
\hline $\mathrm{C}$ & -8.64540700 & 1.78585800 & -4.41060000 \\
\hline $\mathrm{H}$ & -8.48480800 & 2.82637300 & -4.74253000 \\
\hline $\mathrm{H}$ & -7.74517400 & 1.20453600 & -4.67889200 \\
\hline $\mathrm{C}$ & -5.33951000 & 3.88151000 & 1.89375200 \\
\hline
\end{tabular}




\begin{tabular}{|c|c|c|c|}
\hline $\mathrm{H}$ & -6.22454300 & 4.40022400 & 2.30746900 \\
\hline $\mathrm{H}$ & -5.39347700 & 2.81922000 & 2.18562800 \\
\hline $\mathrm{C}$ & -8.01163300 & -4.49089200 & -1.20601600 \\
\hline $\mathrm{H}$ & -8.70416100 & -4.19229100 & -2.01538300 \\
\hline $\mathrm{H}$ & -8.62837900 & -4.75455800 & -0.32923100 \\
\hline $\mathrm{C}$ & -9.91979000 & -0.10922500 & 3.69318600 \\
\hline $\mathrm{H}$ & -10.36804100 & -1.10489600 & 3.52993700 \\
\hline $\mathrm{H}$ & -9.20624700 & -0.19417600 & 4.53242900 \\
\hline $\mathrm{C}$ & -6.94002200 & -3.99130700 & 1.93149900 \\
\hline $\mathrm{H}$ & -7.11539300 & -3.99876600 & 3.02028900 \\
\hline $\mathrm{H}$ & -7.47420500 & -4.87735200 & 1.55185100 \\
\hline $\mathrm{C}$ & -11.01359000 & 0.91180200 & 4.02754500 \\
\hline $\mathrm{H}$ & -10.57059700 & 1.89085100 & 4.25389700 \\
\hline $\mathrm{H}$ & -11.67591000 & 1.03852400 & 3.15940700 \\
\hline $\mathrm{C}$ & 11.37555900 & -0.88842800 & -3.62567300 \\
\hline $\mathrm{H}$ & 10.95308000 & -1.87508200 & -3.85819900 \\
\hline $\mathrm{H}$ & 11.99051300 & -0.99396500 & -2.72065400 \\
\hline $\mathrm{C}$ & -4.04533300 & 4.45374300 & 2.45806200 \\
\hline $\mathrm{H}$ & -3.19207400 & 3.88286400 & 2.06634000 \\
\hline $\mathrm{H}$ & -4.03845300 & 4.38262200 & 3.55418900 \\
\hline $\mathrm{C}$ & 3.87062700 & -3.45913100 & -2.54932600 \\
\hline $\mathrm{H}$ & 3.05328500 & -3.11918700 & -1.89869400 \\
\hline $\mathrm{H}$ & 3.71767600 & -3.03372500 & -3.55051900 \\
\hline $\mathrm{C}$ & 10.08429200 & -2.04735400 & 5.25498800 \\
\hline $\mathrm{H}$ & 9.75586200 & -1.89547700 & 6.29113400 \\
\hline $\mathrm{H}$ & 10.44591600 & -1.08194000 & 4.86982500 \\
\hline $\mathrm{C}$ & -9.84251500 & 1.20069300 & -5.14434400 \\
\hline $\mathrm{H}$ & -9.53948900 & 0.91985600 & -6.16100300 \\
\hline $\mathrm{H}$ & -10.19163800 & 0.29165700 & -4.63188200 \\
\hline $\mathrm{C}$ & -7.17068500 & -5.69606000 & -1.63334800 \\
\hline
\end{tabular}




\begin{tabular}{|c|c|c|c|}
\hline $\mathrm{H}$ & -6.60942100 & -5.46949100 & -2.54962600 \\
\hline $\mathrm{H}$ & -6.44006500 & -5.93044100 & -0.84624900 \\
\hline $\mathrm{C}$ & 7.42651300 & 5.80276000 & 1.81060300 \\
\hline $\mathrm{H}$ & 6.82143600 & 5.55935000 & 2.69416100 \\
\hline $\mathrm{H}$ & 6.73674300 & 6.09728400 & 1.00666800 \\
\hline $\mathrm{N}$ & -7.99196600 & -6.88816100 & -1.87717400 \\
\hline $\mathrm{C}$ & -8.33177800 & -7.38987400 & -3.07718700 \\
\hline $\mathrm{C}$ & -8.55766000 & -7.68716000 & -0.89680800 \\
\hline $\mathrm{H}$ & -8.02970300 & -6.98712000 & -4.04174200 \\
\hline $\mathrm{C}$ & -9.24423900 & -8.68256900 & -1.53479100 \\
\hline $\mathrm{H}$ & -8.41712000 & -7.49514200 & 0.16336300 \\
\hline $\mathrm{H}$ & -9.81780500 & -9.51794800 & -1.13840400 \\
\hline $\mathrm{N}$ & 8.27835000 & 6.95293500 & 2.13600400 \\
\hline $\mathrm{C}$ & 8.90675400 & 7.77473700 & 1.21457000 \\
\hline $\mathrm{C}$ & 8.59075200 & 7.38677800 & 3.36953800 \\
\hline $\mathrm{C}$ & 9.60304600 & 8.71471200 & 1.92228200 \\
\hline $\mathrm{H}$ & 8.79808800 & 7.63703600 & 0.14226700 \\
\hline $\mathrm{H}$ & 8.23961500 & 6.95137100 & 4.30284400 \\
\hline $\mathrm{H}$ & 10.21876100 & 9.54637600 & 1.58589500 \\
\hline $\mathrm{N}$ & -10.97197900 & 2.13165300 & -5.29535900 \\
\hline $\mathrm{C}$ & -11.60402800 & 2.42276200 & -6.49298800 \\
\hline $\mathrm{C}$ & -11.58580100 & 2.81344100 & -4.31275700 \\
\hline $\mathrm{C}$ & -12.61483200 & 3.29872300 & -6.21133000 \\
\hline $\mathrm{H}$ & -11.29165900 & 1.98785600 & -7.43941100 \\
\hline $\mathrm{H}$ & -11.30401800 & 2.78624500 & -3.26319000 \\
\hline $\mathrm{H}$ & -13.34524800 & 3.77272600 & -6.86404200 \\
\hline $\mathrm{N}$ & 11.20988400 & -2.99319200 & 5.31658300 \\
\hline $\mathrm{C}$ & 11.81067500 & -3.59334800 & 4.27482300 \\
\hline $\mathrm{C}$ & 11.85325100 & -3.38302400 & 6.47945400 \\
\hline $\mathrm{H}$ & 11.51777000 & -3.47651900 & 3.23432100 \\
\hline
\end{tabular}




\begin{tabular}{|c|c|c|c|}
\hline $\mathrm{C}$ & 12.85729100 & -4.23638700 & 6.11609200 \\
\hline $\mathrm{H}$ & 11.55186200 & -3.02663700 & 7.46163700 \\
\hline $\mathrm{H}$ & 13.59157500 & -4.76574600 & 6.72005000 \\
\hline $\mathrm{N}$ & 3.77829900 & -4.91757500 & -2.65493100 \\
\hline $\mathrm{C}$ & 3.36159900 & -5.72754600 & -1.67024000 \\
\hline $\mathrm{C}$ & 4.18729000 & -5.69366300 & -3.72716000 \\
\hline $\mathrm{H}$ & 3.00433100 & -5.40818300 & -0.69193600 \\
\hline $\mathrm{C}$ & 3.99974900 & -6.99902800 & -3.36839100 \\
\hline $\mathrm{H}$ & 4.55917300 & -5.26140200 & -4.65235900 \\
\hline $\mathrm{H}$ & 4.17814900 & -7.92032000 & -3.91837400 \\
\hline $\mathrm{N}$ & -3.84791200 & 5.85912400 & 2.09330500 \\
\hline $\mathrm{C}$ & -4.30019000 & 6.96118200 & 2.80069500 \\
\hline $\mathrm{C}$ & -3.26868300 & 6.28166700 & 0.95917600 \\
\hline $\mathrm{C}$ & -3.97219400 & 8.06659700 & 2.06741700 \\
\hline $\mathrm{H}$ & -4.79924900 & 6.87353100 & 3.76226500 \\
\hline $\mathrm{H}$ & -2.84372700 & 5.64123700 & 0.18828800 \\
\hline $\mathrm{H}$ & -4.13071400 & 9.12414500 & 2.26645500 \\
\hline $\mathrm{N}$ & -11.83567400 & 0.50701000 & 5.17367700 \\
\hline $\mathrm{C}$ & -11.84427100 & 1.08474000 & 6.38888400 \\
\hline $\mathrm{C}$ & -12.77831200 & -0.50986800 & 5.17027900 \\
\hline $\mathrm{H}$ & -11.22631900 & 1.92578100 & 6.69658300 \\
\hline $\mathrm{C}$ & -13.35130000 & -0.53068800 & 6.41102400 \\
\hline $\mathrm{H}$ & -12.97843400 & -1.11795600 & 4.29220100 \\
\hline $\mathrm{H}$ & -14.13634000 & -1.16305000 & 6.82084600 \\
\hline $\mathrm{N}$ & 12.25605300 & -0.49605100 & -4.73230400 \\
\hline $\mathrm{C}$ & 13.18703300 & 0.53082800 & -4.69585400 \\
\hline $\mathrm{C}$ & 12.33547700 & -1.09351100 & -5.93522300 \\
\hline $\mathrm{C}$ & 13.82558200 & 0.53737500 & -5.90436700 \\
\hline $\mathrm{H}$ & 13.33354400 & 1.15561300 & -3.81890600 \\
\hline $\mathrm{H}$ & 11.74325500 & -1.94608300 & -6.26118700 \\
\hline
\end{tabular}




\begin{tabular}{|c|c|c|c|}
\hline $\mathrm{H}$ & 14.62502100 & 1.17147700 & -6.28243900 \\
\hline $\mathrm{N}$ & -3.33045600 & 7.61745300 & 0.92561200 \\
\hline $\mathrm{N}$ & 3.48449100 & -6.99369500 & -2.08323200 \\
\hline $\mathrm{N}$ & -12.75379900 & 0.47190100 & 7.15183600 \\
\hline $\mathrm{N}$ & 13.27860300 & -0.48360300 & -6.65889200 \\
\hline $\mathrm{N}$ & 9.39054100 & 8.45275000 & 3.26280500 \\
\hline $\mathrm{N}$ & -9.08847200 & -8.47666700 & -2.89270100 \\
\hline $\mathrm{N}$ & -12.58309900 & 3.52756400 & -4.84879500 \\
\hline $\mathrm{N}$ & 12.81029800 & -4.35281700 & 4.73975800 \\
\hline $\mathrm{C}$ & 3.12404000 & -8.18453600 & -1.30654300 \\
\hline $\mathrm{H}$ & 4.00957400 & -8.82112000 & -1.17594100 \\
\hline $\mathrm{H}$ & 2.74961900 & -7.86590300 & -0.32679500 \\
\hline $\mathrm{H}$ & 2.34243100 & -8.74707800 & -1.83474600 \\
\hline $\mathrm{C}$ & 13.69498800 & -0.84410200 & -8.02384900 \\
\hline $\mathrm{H}$ & 13.09338200 & -1.69212900 & -8.37127100 \\
\hline $\mathrm{H}$ & 13.54209100 & 0.01513600 & -8.69055300 \\
\hline $\mathrm{H}$ & 14.75677400 & -1.12569800 & -8.01713400 \\
\hline $\mathrm{C}$ & 13.71788300 & -5.18201900 & 3.93721900 \\
\hline $\mathrm{H}$ & 14.75292400 & -4.85021300 & 4.09635900 \\
\hline $\mathrm{H}$ & 13.61817400 & -6.23329500 & 4.23991800 \\
\hline $\mathrm{H}$ & 13.45733500 & -5.07798900 & 2.87754300 \\
\hline $\mathrm{C}$ & 9.94273700 & 9.23896600 & 4.37697500 \\
\hline $\mathrm{H}$ & 11.03969000 & 9.22547800 & 4.32456800 \\
\hline $\mathrm{H}$ & 9.58059000 & 10.27369000 & 4.30761700 \\
\hline $\mathrm{H}$ & 9.61460500 & 8.79857600 & 5.32581700 \\
\hline $\mathrm{C}$ & -2.79192700 & 8.47268400 & -0.13702600 \\
\hline $\mathrm{H}$ & -2.34889200 & 7.83485400 & -0.91051900 \\
\hline $\mathrm{H}$ & -3.60176200 & 9.07510700 & -0.57037700 \\
\hline $\mathrm{H}$ & -2.02331100 & 9.13757700 & 0.27956600 \\
\hline $\mathrm{C}$ & -13.09327700 & 0.81349500 & 8.54272900 \\
\hline
\end{tabular}




\begin{tabular}{|c|c|c|c|}
\hline $\mathrm{H}$ & -12.91437100 & -0.05829100 & 9.18626400 \\
\hline $\mathrm{H}$ & -12.46518000 & 1.64970900 & 8.87143800 \\
\hline $\mathrm{H}$ & -14.15088400 & 1.10546000 & 8.59688900 \\
\hline $\mathrm{C}$ & -13.50544200 & 4.41219600 & -4.12574000 \\
\hline $\mathrm{H}$ & -13.41926100 & 5.43326600 & -4.52147900 \\
\hline $\mathrm{H}$ & -14.53516400 & 4.05264300 & -4.25705100 \\
\hline $\mathrm{H}$ & -13.24731700 & 4.40737400 & -3.06038900 \\
\hline $\mathrm{C}$ & -9.65227300 & -9.33205100 & -3.94864300 \\
\hline $\mathrm{H}$ & -9.25512200 & -10.35075500 & -3.84328600 \\
\hline $\mathrm{H}$ & -10.74674400 & -9.34942300 & -3.85858900 \\
\hline $\mathrm{H}$ & -9.37032700 & -8.92824300 & -4.92811000 \\
\hline $\mathrm{C}$ & -4.68640700 & 0.13202300 & 0.51227400 \\
\hline $\mathrm{C}$ & -3.74781100 & 0.51188500 & 1.45432000 \\
\hline $\mathrm{C}$ & -2.35977200 & 0.41929400 & 1.18661500 \\
\hline $\mathrm{C}$ & -1.91930200 & -0.08931100 & -0.07324100 \\
\hline $\mathrm{C}$ & -2.90458800 & -0.47790800 & -1.01279300 \\
\hline $\mathrm{C}$ & -4.25635700 & -0.36817300 & -0.73185900 \\
\hline $\mathrm{C}$ & -1.39410000 & 0.84423900 & 2.16085800 \\
\hline $\mathrm{C}$ & -0.48905600 & -0.15512800 & -0.34842800 \\
\hline $\mathrm{C}$ & 0.43220400 & 0.31473000 & 0.63766200 \\
\hline $\mathrm{C}$ & -0.05864100 & 0.80081100 & 1.89600400 \\
\hline $\mathrm{C}$ & 1.82156200 & 0.29837700 & 0.36433200 \\
\hline $\mathrm{H}$ & 2.50943800 & 0.66589100 & 1.12932600 \\
\hline $\mathrm{C}$ & 2.30301200 & -0.16401700 & -0.84691100 \\
\hline $\mathrm{C}$ & 1.39918400 & -0.62890500 & -1.82305500 \\
\hline $\mathrm{C}$ & 0.03625500 & -0.62806200 & -1.57419500 \\
\hline $\mathrm{H}$ & -1.75314800 & 1.20794100 & 3.12702900 \\
\hline $\mathrm{H}$ & -5.75335400 & 0.19654200 & 0.72744800 \\
\hline $\mathrm{H}$ & -4.06250300 & 0.87279300 & 2.43741800 \\
\hline $\mathrm{H}$ & -2.60268500 & -0.86930400 & -1.98386200 \\
\hline
\end{tabular}




$\begin{array}{crrr}\mathrm{H} & -4.99498800 & -0.68321900 & -1.47110600 \\ \mathrm{H} & 0.66501300 & 1.13602100 & 2.64238300 \\ \mathrm{H} & 3.37693700 & -0.15472900 & -1.04926100 \\ \mathrm{H} & 1.76669300 & -0.97446900 & -2.79205300 \\ \mathrm{H} & -0.63773200 & -0.98774600 & -2.35116100\end{array}$

$E, Z-1$

81

$\mathrm{O}$

$\begin{array}{lll}8.88922300 & -0.34537000 & -2.27780400\end{array}$

O

$\begin{array}{lll}6.93135000 & 3.73672200 & 0.93516300\end{array}$

$\mathrm{O}$

$\begin{array}{lll}6.10042400 & -4.16690100 & -0.37081400\end{array}$

$\mathrm{O}$

$\begin{array}{lll}8.45790700 & -0.60655600 & 3.16731700\end{array}$

$\mathrm{N}$

$\begin{array}{lll}-0.35175200 & 2.37685800 & -2.28508900\end{array}$

$\mathrm{N}$

$\begin{array}{lll}0.28404600 & 2.64835400 & -1.24262500\end{array}$

$\mathrm{C}$

$\begin{array}{lll}-2.43533000 & 2.25328100 & -3.42460300\end{array}$

$\mathrm{H}$

$\begin{array}{lll}-1.84991800 & 1.95556000 & -4.29620400\end{array}$

$\mathrm{C}$

$\begin{array}{lll}-3.81035700 & 2.47345400 & -3.51658800\end{array}$

$\mathrm{H}$

$\begin{array}{lll}-4.31003400 & 2.34399400 & -4.48021100\end{array}$

$\mathrm{C}$

C

$\mathrm{H}$

$\mathrm{C}$

$\mathrm{H}$

$\mathrm{C}$

C

C

$\mathrm{H}$

C

$\mathrm{H}$

$\begin{array}{lll}-4.54329100 & 2.91078600 & -2.40562600\end{array}$

$\begin{array}{lll}-3.86967400 & 3.06332300 & -1.17608200\end{array}$

$\begin{array}{lll}-4.43021700 & 3.39388900 & -0.29802700\end{array}$

$\begin{array}{lll}-2.49819600 & 2.84513100 & -1.07606400\end{array}$

$\begin{array}{lll}-1.96412400 & 3.00101300 & -0.13803500\end{array}$

$\begin{array}{lll}-1.75983600 & 2.46544500 & -2.21474200\end{array}$

$\begin{array}{lll}1.69250800 & 2.71349700 & -1.40485100\end{array}$

$\begin{array}{lll}2.35436800 & 2.54325900 & -2.63360300\end{array}$

$\begin{array}{lll}1.76644900 & 2.26024300 & -3.50667700\end{array}$

$\begin{array}{lll}3.72059800 & 2.79362300 & -2.72844900\end{array}$

$\begin{array}{lll}4.21114000 & 2.70536500 & -3.70204100\end{array}$

C

$\begin{array}{lll}4.46915600 & 3.21032000 & -1.61333200\end{array}$ 


\begin{tabular}{|c|c|c|c|}
\hline $\mathrm{C}$ & 3.81518400 & 3.31100000 & -0.37428500 \\
\hline $\mathrm{H}$ & 4.38166500 & 3.61688500 & 0.50833400 \\
\hline $\mathrm{C}$ & 2.44277300 & 3.07204100 & -0.27325500 \\
\hline $\mathrm{H}$ & 1.91499100 & 3.19831000 & 0.67438000 \\
\hline $\mathrm{C}$ & 5.92852800 & 3.62043800 & -1.77791500 \\
\hline $\mathrm{H}$ & 6.06820000 & 4.60035500 & -1.29794800 \\
\hline $\mathrm{H}$ & 6.13067200 & 3.76358400 & -2.84915700 \\
\hline $\mathrm{C}$ & 6.93908800 & 2.64078800 & -1.21608700 \\
\hline $\mathrm{C}$ & 7.46893500 & 1.61913200 & -2.01745000 \\
\hline $\mathrm{H}$ & 7.16587600 & 1.57476700 & -3.06514400 \\
\hline $\mathrm{C}$ & 8.34145100 & 0.65690600 & -1.49912200 \\
\hline $\mathrm{C}$ & 8.65320600 & 0.63867000 & -0.12478400 \\
\hline $\mathrm{C}$ & 8.16488700 & 1.68939800 & 0.66173100 \\
\hline $\mathrm{H}$ & 8.40283200 & 1.71705500 & 1.72682300 \\
\hline $\mathrm{C}$ & 7.35589500 & 2.69395400 & 0.12393200 \\
\hline $\mathrm{C}$ & 9.37865400 & -0.54173800 & 0.50326200 \\
\hline $\mathrm{H}$ & 10.03345700 & -1.01484000 & -0.23912400 \\
\hline $\mathrm{H}$ & 9.99732300 & -0.19249500 & 1.33951300 \\
\hline $\mathrm{C}$ & 8.34183000 & -1.54031900 & 0.99298800 \\
\hline $\mathrm{C}$ & 7.77880500 & -2.47239500 & 0.11135500 \\
\hline $\mathrm{H}$ & 8.19887500 & -2.55478600 & -0.89262400 \\
\hline $\mathrm{C}$ & 6.65697400 & -3.22929100 & 0.46494100 \\
\hline $\mathrm{C}$ & 6.01161400 & -3.00667400 & 1.69385400 \\
\hline $\mathrm{C}$ & 6.62987100 & -2.14467300 & 2.60909600 \\
\hline $\mathrm{H}$ & 6.14676400 & -2.01221800 & 3.57758000 \\
\hline $\mathrm{C}$ & 7.80066700 & -1.44602100 & 2.28947600 \\
\hline $\mathrm{C}$ & 9.26616000 & -0.07531000 & -3.61078000 \\
\hline $\mathrm{H}$ & 8.43871200 & 0.35380800 & -4.20075100 \\
\hline $\mathrm{H}$ & 9.51796500 & -1.04763000 & -4.05842500 \\
\hline $\mathrm{C}$ & 7.82257300 & 4.83251300 & 1.02002600 \\
\hline
\end{tabular}




\begin{tabular}{|c|c|c|c|}
\hline $\mathrm{H}$ & 8.75542500 & 4.54513700 & 1.54083900 \\
\hline $\mathrm{H}$ & 8.10359400 & 5.19767100 & 0.01474900 \\
\hline $\mathrm{C}$ & 6.93959900 & -4.94996000 & -1.18807500 \\
\hline $\mathrm{H}$ & 7.12832400 & -4.46145700 & -2.16222600 \\
\hline $\mathrm{H}$ & 7.91625200 & -5.12842900 & -0.70344700 \\
\hline $\mathrm{C}$ & 8.06686600 & -0.54199100 & 4.52376200 \\
\hline $\mathrm{H}$ & 8.52920100 & 0.37091100 & 4.92666600 \\
\hline $\mathrm{H}$ & 6.97483800 & -0.42934600 & 4.62256900 \\
\hline $\mathrm{C}$ & 4.64441300 & -3.58427000 & 1.96953600 \\
\hline $\mathrm{H}$ & 4.47751800 & -3.61821700 & 3.05962500 \\
\hline $\mathrm{H}$ & 4.58979800 & -4.62163400 & 1.61020300 \\
\hline $\mathrm{O}$ & -7.89752900 & 0.39303000 & 3.17725000 \\
\hline $\mathrm{O}$ & -6.59180400 & -4.33939300 & 0.59726800 \\
\hline $\mathrm{O}$ & -6.89804200 & 4.07603200 & 0.08141600 \\
\hline $\mathrm{O}$ & -8.93087400 & -0.67989900 & -1.92992500 \\
\hline $\mathrm{N}$ & 0.51993500 & -0.49451600 & -0.62327600 \\
\hline $\mathrm{N}$ & -0.71454700 & -0.60417000 & -0.68749800 \\
\hline $\mathrm{C}$ & 2.59835800 & -0.67243600 & 0.52537700 \\
\hline $\mathrm{H}$ & 2.70471900 & 0.39408400 & 0.32000000 \\
\hline $\mathrm{C}$ & 3.62380700 & -1.39228400 & 1.13172000 \\
\hline $\mathrm{H}$ & 4.53569400 & -0.86855200 & 1.42394000 \\
\hline $\mathrm{C}$ & 3.51975800 & -2.77975300 & 1.32007800 \\
\hline $\mathrm{C}$ & 2.34030700 & -3.41540000 & 0.89610200 \\
\hline $\mathrm{H}$ & 2.24201600 & -4.49812400 & 1.01372300 \\
\hline $\mathrm{C}$ & 1.28910500 & -2.69937700 & 0.32141800 \\
\hline $\mathrm{H}$ & 0.39356100 & -3.22480500 & -0.01161200 \\
\hline $\mathrm{C}$ & 1.40908500 & -1.31015500 & 0.13335100 \\
\hline $\mathrm{C}$ & -1.54281800 & -1.40991000 & 0.14251800 \\
\hline $\mathrm{C}$ & -1.49378800 & -1.38958500 & 1.54523300 \\
\hline $\mathrm{H}$ & -0.68914800 & -0.85638700 & 2.05456100 \\
\hline
\end{tabular}




\begin{tabular}{|c|c|c|c|}
\hline $\mathrm{C}$ & -2.49853500 & -2.02040700 & 2.28482200 \\
\hline $\mathrm{H}$ & -2.45878400 & -1.97891700 & 3.37692000 \\
\hline $\mathrm{C}$ & -3.55042200 & -2.70337000 & 1.65679300 \\
\hline $\mathrm{C}$ & -3.57508500 & -2.73502900 & 0.25153000 \\
\hline $\mathrm{H}$ & -4.38658600 & -3.25753200 & -0.26013100 \\
\hline $\mathrm{C}$ & -2.60458100 & -2.07629200 & -0.49528000 \\
\hline $\mathrm{H}$ & -2.65462800 & -2.04951300 & -1.58560900 \\
\hline $\mathrm{C}$ & -4.67653400 & -3.34069500 & 2.45991300 \\
\hline $\mathrm{H}$ & -4.75463300 & -4.40753500 & 2.20602900 \\
\hline $\mathrm{H}$ & -4.44278100 & -3.27441700 & 3.53238200 \\
\hline $\mathrm{C}$ & -6.00276800 & -2.66097200 & 2.18077400 \\
\hline $\mathrm{C}$ & -6.34551900 & -1.47688800 & 2.85056900 \\
\hline $\mathrm{H}$ & -5.67262900 & -1.13604200 & 3.63789200 \\
\hline $\mathrm{C}$ & -7.48487400 & -0.74265300 & 2.51037800 \\
\hline $\mathrm{C}$ & -8.27544700 & -1.13287600 & 1.40911000 \\
\hline $\mathrm{C}$ & -7.97620600 & -2.35112500 & 0.79095400 \\
\hline $\mathrm{H}$ & -8.57928300 & -2.66536800 & -0.06243900 \\
\hline $\mathrm{C}$ & -6.88416700 & -3.13089400 & 1.19477800 \\
\hline $\mathrm{C}$ & -9.30794100 & -0.18196000 & 0.82361000 \\
\hline $\mathrm{H}$ & -9.81744300 & 0.36752700 & 1.62597600 \\
\hline $\mathrm{H}$ & -10.06024400 & -0.75424900 & 0.26594300 \\
\hline $\mathrm{C}$ & -8.61553100 & 0.80639800 & -0.10290300 \\
\hline $\mathrm{C}$ & -8.11572400 & 2.02430000 & 0.37232200 \\
\hline $\mathrm{H}$ & -8.31040400 & 2.31876100 & 1.40448500 \\
\hline $\mathrm{C}$ & -7.34597100 & 2.86764200 & -0.43383300 \\
\hline $\mathrm{C}$ & -6.98333300 & 2.48467400 & -1.73442100 \\
\hline $\mathrm{C}$ & -7.53007100 & 1.29251100 & -2.23284100 \\
\hline $\mathrm{H}$ & -7.27021300 & 0.99012300 & -3.24902600 \\
\hline $\mathrm{C}$ & -8.36190600 & 0.48433200 & -1.45252100 \\
\hline $\mathrm{C}$ & -7.17347600 & 0.86775600 & 4.29170700 \\
\hline
\end{tabular}




\begin{tabular}{|c|c|c|c|}
\hline $\mathrm{H}$ & -6.08766900 & 0.85481000 & 4.10259800 \\
\hline $\mathrm{H}$ & -7.47366000 & 1.91778200 & 4.42333700 \\
\hline $\mathrm{C}$ & -7.63793200 & -5.25211600 & 0.34112600 \\
\hline $\mathrm{H}$ & -8.05334400 & -5.11404100 & -0.67423100 \\
\hline $\mathrm{H}$ & -8.46220100 & -5.12496300 & 1.06476200 \\
\hline $\mathrm{C}$ & -7.75907300 & 5.17889300 & -0.12772400 \\
\hline $\mathrm{H}$ & -8.69809200 & 5.06167700 & 0.44566000 \\
\hline $\mathrm{H}$ & -8.03292200 & 5.27716800 & -1.19452900 \\
\hline $\mathrm{C}$ & -9.33478300 & -0.77353900 & -3.27831200 \\
\hline $\mathrm{H}$ & -9.59655300 & -1.82986800 & -3.43746200 \\
\hline $\mathrm{H}$ & -8.52258700 & -0.52753400 & -3.98291900 \\
\hline $\mathrm{C}$ & -6.01012500 & 3.29444900 & -2.56563800 \\
\hline $\mathrm{H}$ & -6.28035600 & 3.19905200 & -3.62718300 \\
\hline $\mathrm{H}$ & -6.11518800 & 4.36044800 & -2.31180200 \\
\hline $\mathrm{C}$ & -6.99527600 & 6.42001400 & 0.33787900 \\
\hline $\mathrm{H}$ & -6.08717800 & 6.55222200 & -0.26555100 \\
\hline $\mathrm{H}$ & -6.68182300 & 6.29499000 & 1.38396400 \\
\hline $\mathrm{C}$ & -10.56803100 & 0.11155200 & -3.54182900 \\
\hline $\mathrm{H}$ & -10.29979300 & 1.17626900 & -3.51909700 \\
\hline $\mathrm{H}$ & -11.31102700 & -0.06040000 & -2.75028000 \\
\hline $\mathrm{C}$ & -7.45642800 & 0.08580200 & 5.57778500 \\
\hline $\mathrm{H}$ & -6.73164100 & 0.37561500 & 6.35040400 \\
\hline $\mathrm{H}$ & -7.34622900 & -0.99484500 & 5.41068200 \\
\hline $\mathrm{C}$ & -7.01457400 & -6.64736900 & 0.47635000 \\
\hline $\mathrm{H}$ & -6.64870400 & -6.79281800 & 1.50131400 \\
\hline $\mathrm{H}$ & -6.15103600 & -6.73206800 & -0.19851900 \\
\hline $\mathrm{C}$ & 8.50451000 & -1.75368500 & 5.35273000 \\
\hline $\mathrm{H}$ & 7.98420900 & -1.73667100 & 6.32037600 \\
\hline $\mathrm{H}$ & 8.23186400 & -2.69001700 & 4.84330000 \\
\hline $\mathrm{C}$ & 6.19292000 & -6.27452600 & -1.38748400 \\
\hline
\end{tabular}




\begin{tabular}{|c|c|c|c|}
\hline $\mathrm{H}$ & 6.02450600 & -6.75106700 & -0.41280400 \\
\hline $\mathrm{H}$ & 5.20876100 & -6.08167800 & -1.83738800 \\
\hline $\mathrm{C}$ & 10.49538800 & 0.85030900 & -3.64889100 \\
\hline $\mathrm{H}$ & 10.25373500 & 1.82585000 & -3.20262500 \\
\hline $\mathrm{H}$ & 11.30920400 & 0.40592600 & -3.06042200 \\
\hline $\mathrm{C}$ & 7.09229300 & 5.92982600 & 1.79682200 \\
\hline $\mathrm{H}$ & 6.19470900 & 6.24845400 & 1.24992000 \\
\hline $\mathrm{H}$ & 6.76534700 & 5.54166100 & 2.77171600 \\
\hline $\mathrm{N}$ & 7.93149600 & 7.11058500 & 2.03127300 \\
\hline $\mathrm{C}$ & 7.85689400 & 8.27787900 & 1.36848300 \\
\hline $\mathrm{C}$ & 8.93397800 & 7.21391700 & 2.98170100 \\
\hline $\mathrm{H}$ & 7.15581000 & 8.50952800 & 0.56924000 \\
\hline $\mathrm{C}$ & 9.46052800 & 8.47122200 & 2.87618300 \\
\hline $\mathrm{H}$ & 9.17845800 & 6.40148100 & 3.66040600 \\
\hline $\mathrm{H}$ & 10.25297500 & 8.95984800 & 3.43929100 \\
\hline $\mathrm{N}$ & 10.99485100 & 1.08057600 & -5.00887100 \\
\hline $\mathrm{C}$ & 10.36674200 & 1.83169800 & -5.98959900 \\
\hline $\mathrm{C}$ & 12.15344500 & 0.61682600 & -5.51101200 \\
\hline $\mathrm{C}$ & 11.17477100 & 1.80864800 & -7.09258100 \\
\hline $\mathrm{H}$ & 9.41509800 & 2.32878900 & -5.82357500 \\
\hline $\mathrm{H}$ & 12.87812100 & 0.00659100 & -4.97541900 \\
\hline $\mathrm{H}$ & 11.05843000 & 2.27407400 & -8.06911800 \\
\hline $\mathrm{N}$ & 6.91028500 & -7.22911700 & -2.24023800 \\
\hline $\mathrm{C}$ & 7.54907300 & -8.33357800 & -1.81639000 \\
\hline $\mathrm{C}$ & 7.01780600 & -7.16705200 & -3.61969500 \\
\hline $\mathrm{H}$ & 7.62611300 & -8.66389300 & -0.78229500 \\
\hline $\mathrm{H}$ & 6.56112600 & -6.37202200 & -4.20292500 \\
\hline $\mathrm{N}$ & -11.21241500 & -0.17013400 & -4.82957200 \\
\hline $\mathrm{C}$ & -12.05900900 & -1.23704100 & -5.08580500 \\
\hline $\mathrm{C}$ & -11.10790200 & 0.56311500 & -5.95212900 \\
\hline
\end{tabular}




\begin{tabular}{|c|c|c|c|}
\hline $\mathrm{C}$ & -12.46207900 & -1.12779000 & -6.38744100 \\
\hline $\mathrm{H}$ & -12.32207800 & -1.96698600 & -4.32497900 \\
\hline $\mathrm{H}$ & -10.52475200 & 1.47475800 & -6.06279400 \\
\hline $\mathrm{H}$ & -13.13444100 & -1.74691100 & -6.97790900 \\
\hline $\mathrm{N}$ & -7.79535300 & 7.64629600 & 0.24029400 \\
\hline $\mathrm{C}$ & -8.82241200 & 8.01260900 & 1.09475500 \\
\hline $\mathrm{C}$ & -7.65239000 & 8.61105800 & -0.68497400 \\
\hline $\mathrm{C}$ & -9.29396600 & 9.22088300 & 0.66236200 \\
\hline $\mathrm{H}$ & -9.12194800 & 7.40116600 & 1.94139900 \\
\hline $\mathrm{H}$ & -6.91704100 & 8.61577900 & -1.48696400 \\
\hline $\mathrm{H}$ & -10.08394600 & 9.85769500 & 1.05511700 \\
\hline $\mathrm{N}$ & -8.79193400 & 0.33113700 & 6.13918300 \\
\hline $\mathrm{C}$ & -9.05740700 & 1.04076900 & 7.29850300 \\
\hline $\mathrm{C}$ & -9.95584500 & -0.11230900 & 5.63571900 \\
\hline $\mathrm{C}$ & -10.41127000 & 1.01245000 & 7.48607500 \\
\hline $\mathrm{H}$ & -8.27098700 & 1.48911200 & 7.90061600 \\
\hline $\mathrm{H}$ & -10.06573700 & -0.70381900 & 4.73042800 \\
\hline $\mathrm{H}$ & -11.02730600 & 1.43407200 & 8.27770100 \\
\hline $\mathrm{N}$ & -7.94527200 & -7.73905900 & 0.16751500 \\
\hline $\mathrm{C}$ & -8.34848100 & -8.12920400 & -1.09916400 \\
\hline $\mathrm{C}$ & -8.52291800 & -8.55748200 & 1.06460700 \\
\hline $\mathrm{C}$ & -9.18332300 & -9.20087400 & -0.94287800 \\
\hline $\mathrm{H}$ & -7.99599800 & -7.63421200 & -1.99998900 \\
\hline $\mathrm{H}$ & -8.38520800 & -8.51443500 & 2.14324800 \\
\hline $\mathrm{H}$ & -9.70383700 & -9.81047000 & -1.67847200 \\
\hline $\mathrm{N}$ & 9.94603800 & -1.78736500 & 5.63776200 \\
\hline $\mathrm{C}$ & 10.49223100 & -1.89360100 & 6.86147300 \\
\hline $\mathrm{C}$ & 10.96710300 & -1.77412100 & 4.70215400 \\
\hline $\mathrm{H}$ & 9.94375500 & -1.94046800 & 7.80070700 \\
\hline $\mathrm{C}$ & 12.14296600 & -1.87216900 & 5.39503700 \\
\hline
\end{tabular}




\begin{tabular}{|c|c|c|c|}
\hline $\mathrm{H}$ & 10.76430800 & -1.69097500 & 3.63923900 \\
\hline $\mathrm{H}$ & 13.17358800 & -1.89868300 & 5.04878200 \\
\hline $\mathrm{N}$ & 11.82184600 & -1.94645400 & 6.73747600 \\
\hline $\mathrm{N}$ & -9.27622800 & -9.44919600 & 0.41383100 \\
\hline $\mathrm{N}$ & -11.85667200 & 0.00118500 & -6.90635000 \\
\hline $\mathrm{N}$ & -8.54922100 & 9.57349100 & -0.44748300 \\
\hline $\mathrm{N}$ & -10.94927000 & 0.28824100 & 6.43889800 \\
\hline $\mathrm{N}$ & 8.77318600 & 9.11532300 & 1.86450000 \\
\hline $\mathrm{N}$ & 12.28178900 & 1.04687500 & -6.76984500 \\
\hline $\mathrm{C}$ & -8.71187500 & 10.81737900 & -1.21554700 \\
\hline $\mathrm{H}$ & -9.73393500 & 10.86902000 & -1.61416600 \\
\hline $\mathrm{H}$ & -8.52559300 & 11.67799600 & -0.55883000 \\
\hline $\mathrm{H}$ & -7.99340800 & 10.82600200 & -2.04360400 \\
\hline $\mathrm{C}$ & -12.37667500 & -0.01281300 & 6.27093900 \\
\hline $\mathrm{H}$ & -12.72587600 & -0.61749100 & 7.11925700 \\
\hline $\mathrm{H}$ & -12.94670400 & 0.92494300 & 6.22875100 \\
\hline $\mathrm{H}$ & -12.51913700 & -0.57237500 & 5.33908800 \\
\hline $\mathrm{C}$ & 9.00579300 & 10.50198700 & 1.43237500 \\
\hline $\mathrm{H}$ & 8.81901100 & 11.18229000 & 2.27436300 \\
\hline $\mathrm{H}$ & 10.04455300 & 10.60873900 & 1.09218500 \\
\hline $\mathrm{H}$ & 8.32288000 & 10.74166900 & 0.60892400 \\
\hline $\mathrm{C}$ & 13.41903400 & 0.78057300 & -7.66550800 \\
\hline $\mathrm{H}$ & 13.06134400 & 0.26144000 & -8.56478700 \\
\hline $\mathrm{H}$ & 13.89040000 & 1.73183800 & -7.94743200 \\
\hline $\mathrm{H}$ & 14.14915000 & 0.15148900 & -7.14289000 \\
\hline $\mathrm{C}$ & -12.04116300 & 0.50587300 & -8.27588000 \\
\hline $\mathrm{H}$ & -11.72468600 & -0.26269400 & -8.99368800 \\
\hline $\mathrm{H}$ & -13.10075000 & 0.74865000 & -8.43462600 \\
\hline $\mathrm{H}$ & -11.43403000 & 1.40850300 & -8.41161200 \\
\hline $\mathrm{C}$ & -10.05550200 & -10.53707800 & 1.02570900 \\
\hline
\end{tabular}




$\begin{array}{lrrr}\mathrm{H} & -11.11437000 & -10.42357400 & 0.75739500 \\ \mathrm{H} & -9.68088300 & -11.50308200 & 0.66079000 \\ \mathrm{H} & -9.94511200 & -10.49003400 & 2.11546300 \\ \mathrm{C} & 12.78809100 & -2.07341200 & 7.83994400 \\ \mathrm{H} & 13.36671600 & -2.99831100 & 7.71286700 \\ \mathrm{H} & 13.46332500 & -1.20729900 & 7.83635900 \\ \mathrm{H} & 12.24498300 & -2.11065000 & 8.79165400 \\ \mathrm{C} & 7.73723500 & -8.26046600 & -4.01612600 \\ \mathrm{H} & 8.03440900 & -8.59544000 & -5.00756000 \\ \mathrm{~N} & 8.05672800 & -8.97238500 & -2.87497200 \\ \mathrm{C} & 8.80550700 & -10.23850000 & -2.84540700 \\ \mathrm{H} & 8.91332800 & -10.56844600 & -1.80547300 \\ \mathrm{H} & 8.25673000 & -10.99977700 & -3.41633000 \\ \mathrm{H} & 9.79896800 & -10.08617500 & -3.28799400\end{array}$

\section{phenanthrene}

01

C

C

C

C

C

C

C

C

C

C

C

$\mathrm{H}$

\begin{tabular}{lrr}
0.00000000 & 3.56254200 & -0.29987600 \\
0.00000000 & 2.84025600 & 0.87900600 \\
0.00000000 & 1.42430400 & 0.86874800 \\
0.00000000 & 0.72852900 & -0.37819600 \\
0.00000000 & 1.49668100 & -1.56734300 \\
0.00000000 & 2.88088500 & -1.53318400 \\
0.00000000 & 0.68090200 & 2.09752600 \\
0.00000000 & -0.72852900 & -0.37819600 \\
0.00000000 & -1.42430400 & 0.86874800 \\
0.00000000 & -0.68090200 & 2.09752600 \\
0.00000000 & -2.84025600 & 0.87900600 \\
\hline 0.00000000 & -3.35569100 & 1.84296100 \\
0.00000000 & -3.56254200 & -0.29987600
\end{tabular}




$\begin{array}{llrr}\mathrm{C} & 0.00000000 & -2.88088500 & -1.53318400 \\ \mathrm{C} & 0.00000000 & -1.49668100 & -1.56734300 \\ \mathrm{H} & 0.00000000 & 1.23664700 & 3.03863000 \\ \mathrm{H} & 0.00000000 & 4.65475100 & -0.27788100 \\ \mathrm{H} & 0.00000000 & 3.35569100 & 1.84296100 \\ \mathrm{H} & 0.00000000 & 0.99605300 & -2.53570900 \\ \mathrm{H} & 0.00000000 & 3.44620600 & -2.46808900 \\ \mathrm{H} & 0.00000000 & -1.23664700 & 3.03863000 \\ \mathrm{H} & 0.00000000 & -4.65475100 & -0.27788100 \\ \mathrm{H} & 0.00000000 & -3.44620600 & -2.46808900 \\ \mathrm{H} & 0.00000000 & -0.99605300 & -2.53570900\end{array}$

\section{Z,Z-1}

81

$\begin{array}{lrrr}\text { O } & 6.46275200 & -4.20393300 & -0.38803300 \\ \mathrm{O} & 8.31463700 & 0.07965500 & 2.59872600 \\ \mathrm{C} & 8.24995400 & -1.08310500 & 0.53599700 \\ \mathrm{C} & 7.83373900 & -2.20537200 & -0.19183400 \\ \mathrm{H} & 8.22582900 & -2.35067200 & -1.19946700 \\ \mathrm{C} & 6.87690500 & -3.09511900 & 0.31179700 \\ \mathrm{C} & 6.23537100 & -2.82608300 & 1.53246500 \\ \mathrm{C} & 6.72927200 & -1.76765700 & 2.30745000 \\ \mathrm{H} & 6.25725700 & -1.59210000 & 3.27446200 \\ \mathrm{C} & 7.75666200 & -0.93511500 & 1.84866900 \\ \mathrm{C} & 7.42565900 & -5.03424600 & -1.00225500 \\ \mathrm{H} & 7.58770900 & -4.74920500 & -2.05840500 \\ \mathrm{H} & 8.39735300 & -4.97231800 & -0.48143300 \\ \mathrm{C} & 7.96476600 & 0.23604600 & 3.95958200 \\ \mathrm{H} & 8.32783200 & 1.23296700 & 4.24906100 \\ \mathrm{H} & 6.87056600 & 0.23105700 & 4.09429100\end{array}$




\begin{tabular}{|c|c|c|c|}
\hline $\mathrm{C}$ & 4.97310400 & -3.55497200 & 1.92566000 \\
\hline $\mathrm{H}$ & 4.82337700 & -3.47005500 & 3.01535300 \\
\hline $\mathrm{H}$ & 5.06525100 & -4.62796100 & 1.70381200 \\
\hline $\mathrm{O}$ & -7.58474000 & 0.68605400 & 2.61238700 \\
\hline $\mathrm{O}$ & -6.95060800 & -4.35738800 & 0.39920600 \\
\hline $\mathrm{N}$ & 0.51367400 & -1.52364300 & -1.09711300 \\
\hline $\mathrm{N}$ & -0.70338700 & -1.76277900 & -1.15553800 \\
\hline $\mathrm{C}$ & 2.60868300 & -1.24699000 & -0.02509300 \\
\hline $\mathrm{H}$ & 2.62024000 & -0.26394200 & -0.49455700 \\
\hline $\mathrm{C}$ & 3.71399300 & -1.71013000 & 0.67880600 \\
\hline $\mathrm{H}$ & 4.58469900 & -1.06053300 & 0.77835200 \\
\hline $\mathrm{C}$ & 3.73944700 & -3.01130900 & 1.20466600 \\
\hline $\mathrm{C}$ & 2.59583900 & -3.81270600 & 1.03807200 \\
\hline $\mathrm{H}$ & 2.59384100 & -4.83409100 & 1.42875700 \\
\hline $\mathrm{C}$ & 1.46308000 & -3.34248700 & 0.37063000 \\
\hline $\mathrm{H}$ & 0.60000500 & -3.99639500 & 0.23981700 \\
\hline $\mathrm{C}$ & 1.46372300 & -2.04721300 & -0.18042500 \\
\hline $\mathrm{C}$ & -1.54259200 & -2.37882700 & -0.18822200 \\
\hline $\mathrm{C}$ & -1.53922000 & -2.05409500 & 1.17859400 \\
\hline $\mathrm{H}$ & -0.73315600 & -1.44953900 & 1.59392200 \\
\hline $\mathrm{C}$ & -2.61597600 & -2.44443900 & 1.98069100 \\
\hline $\mathrm{H}$ & -2.61857800 & -2.15776900 & 3.03599000 \\
\hline $\mathrm{C}$ & -3.69510500 & -3.17385500 & 1.45956200 \\
\hline $\mathrm{C}$ & -3.66371400 & -3.53183800 & 0.10068000 \\
\hline $\mathrm{H}$ & -4.49430200 & -4.09968700 & -0.32460600 \\
\hline $\mathrm{C}$ & -2.61778500 & -3.11975400 & -0.71545400 \\
\hline $\mathrm{H}$ & -2.62283100 & -3.33620000 & -1.78586900 \\
\hline $\mathrm{C}$ & -4.91493300 & -3.51127500 & 2.30587800 \\
\hline $\mathrm{H}$ & -5.15736500 & -4.57691900 & 2.18588400 \\
\hline $\mathrm{H}$ & -4.68288500 & -3.34815000 & 3.36829200 \\
\hline
\end{tabular}




\begin{tabular}{|c|c|c|c|}
\hline $\mathrm{C}$ & -6.11582800 & -2.66740400 & 1.91335800 \\
\hline $\mathrm{C}$ & -6.29436800 & -1.39041100 & 2.47144100 \\
\hline $\mathrm{H}$ & -5.59248300 & -1.07885600 & 3.24529400 \\
\hline $\mathrm{C}$ & -7.31465800 & -0.53649500 & 2.04102700 \\
\hline $\mathrm{C}$ & -8.14208000 & -0.89964300 & 0.95519400 \\
\hline $\mathrm{C}$ & -7.99954000 & -2.19285200 & 0.44538900 \\
\hline $\mathrm{H}$ & -8.63551200 & -2.51868400 & -0.37967800 \\
\hline $\mathrm{C}$ & -7.03268900 & -3.08042200 & 0.93660900 \\
\hline $\mathrm{C}$ & -6.76001000 & 1.19689800 & 3.63725200 \\
\hline $\mathrm{H}$ & -5.69316900 & 1.03645600 & 3.41078400 \\
\hline $\mathrm{H}$ & -6.93487500 & 2.28283100 & 3.65421800 \\
\hline $\mathrm{C}$ & -7.77818200 & -5.32948000 & 1.01227600 \\
\hline $\mathrm{H}$ & -8.84569300 & -5.05796700 & 0.91044200 \\
\hline $\mathrm{H}$ & -7.55969500 & -5.41737800 & 2.09274700 \\
\hline $\mathrm{C}$ & -7.06510000 & 0.60137500 & 5.01509000 \\
\hline $\mathrm{H}$ & -6.28972400 & 0.91237100 & 5.72831000 \\
\hline $\mathrm{H}$ & -7.05757100 & -0.49656600 & 4.97801500 \\
\hline $\mathrm{C}$ & -7.49215800 & -6.65349700 & 0.30042700 \\
\hline $\mathrm{H}$ & -6.44435000 & -6.94612600 & 0.45049400 \\
\hline $\mathrm{H}$ & -7.65041400 & -6.53441800 & -0.78093200 \\
\hline $\mathrm{C}$ & 8.56612800 & -0.82424200 & 4.88837600 \\
\hline $\mathrm{H}$ & 8.07908400 & -0.75666900 & 5.87106500 \\
\hline $\mathrm{H}$ & 8.38125700 & -1.83442400 & 4.49410100 \\
\hline $\mathrm{C}$ & 6.85739600 & -6.45711500 & -0.91570500 \\
\hline $\mathrm{H}$ & 6.74763400 & -6.74805000 & 0.13730100 \\
\hline $\mathrm{H}$ & 5.85720400 & -6.47949500 & -1.37115800 \\
\hline $\mathrm{N}$ & 7.68789000 & -7.46782600 & -1.58049400 \\
\hline $\mathrm{C}$ & 8.45362300 & -8.38554400 & -0.96390400 \\
\hline $\mathrm{C}$ & 7.78283200 & -7.67103600 & -2.94764600 \\
\hline $\mathrm{H}$ & 8.57084300 & -8.49384900 & 0.11258700 \\
\hline
\end{tabular}




\begin{tabular}{|c|c|c|c|}
\hline $\mathrm{H}$ & 7.23290500 & -7.06789700 & -3.66488400 \\
\hline $\mathrm{N}$ & -8.35390000 & 1.02732400 & 5.57722300 \\
\hline $\mathrm{C}$ & -8.52143100 & 1.94353900 & 6.60210500 \\
\hline $\mathrm{C}$ & -9.56385800 & 0.57384600 & 5.21013500 \\
\hline $\mathrm{C}$ & -9.86330800 & 2.02935300 & 6.84919200 \\
\hline $\mathrm{H}$ & -7.68215700 & 2.43972900 & 7.08303600 \\
\hline $\mathrm{H}$ & -9.75190700 & -0.16131000 & 4.43175000 \\
\hline $\mathrm{H}$ & -10.41463900 & 2.61635100 & 7.58088100 \\
\hline $\mathrm{N}$ & -8.34464500 & -7.75044800 & 0.77284400 \\
\hline $\mathrm{C}$ & -9.69210500 & -7.90768400 & 0.49070700 \\
\hline $\mathrm{C}$ & -7.94835500 & -8.79069000 & 1.52719800 \\
\hline $\mathrm{C}$ & -10.09703600 & -9.06603700 & 1.09334400 \\
\hline $\mathrm{H}$ & -10.24522700 & -7.20228200 & -0.12300900 \\
\hline $\mathrm{H}$ & -6.93878900 & -8.95732800 & 1.89726900 \\
\hline $\mathrm{H}$ & -11.06798100 & -9.55679700 & 1.11106900 \\
\hline $\mathrm{N}$ & 10.01140000 & -0.67630100 & 5.11123600 \\
\hline $\mathrm{C}$ & 10.60927900 & -0.63818900 & 6.31495700 \\
\hline $\mathrm{C}$ & 10.99204800 & -0.63324400 & 4.13402200 \\
\hline $\mathrm{H}$ & 10.10244200 & -0.67063900 & 7.27799100 \\
\hline $\mathrm{C}$ & 12.19588000 & -0.56318300 & 4.78095700 \\
\hline $\mathrm{H}$ & 10.74342200 & -0.65180000 & 3.07777000 \\
\hline $\mathrm{H}$ & 13.21099300 & -0.51412000 & 4.39355900 \\
\hline $\mathrm{N}$ & 11.93206800 & -0.56870600 & 6.13786500 \\
\hline $\mathrm{N}$ & -8.99318800 & -9.59835900 & 1.73264900 \\
\hline $\mathrm{N}$ & -10.49211700 & 1.16676800 & 5.97087200 \\
\hline $\mathrm{C}$ & -11.93940900 & 0.92091900 & 5.92474400 \\
\hline $\mathrm{H}$ & -12.26823000 & 0.48977100 & 6.88042200 \\
\hline $\mathrm{H}$ & -12.46680100 & 1.86776400 & 5.74785800 \\
\hline $\mathrm{H}$ & -12.15959800 & 0.21919000 & 5.11181300 \\
\hline $\mathrm{C}$ & -8.97937100 & -10.86109500 & 2.48765300 \\
\hline
\end{tabular}




\begin{tabular}{|c|c|c|c|}
\hline $\mathrm{H}$ & -9.70544600 & -10.80247600 & 3.30949800 \\
\hline $\mathrm{H}$ & -9.24371900 & -11.68938900 & 1.81622500 \\
\hline $\mathrm{H}$ & -7.97501300 & -11.02558800 & 2.89513000 \\
\hline $\mathrm{C}$ & 12.94529300 & -0.52018200 & 7.20431200 \\
\hline $\mathrm{H}$ & 13.60486300 & -1.39449500 & 7.12047400 \\
\hline $\mathrm{H}$ & 13.53413500 & 0.40178700 & 7.10805300 \\
\hline $\mathrm{H}$ & 12.44280600 & -0.53424300 & 8.17869800 \\
\hline $\mathrm{C}$ & 8.62466900 & -8.73151100 & -3.13893500 \\
\hline $\mathrm{H}$ & 8.95601700 & -9.22111400 & -4.05228500 \\
\hline $\mathrm{N}$ & 9.02915200 & -9.16002000 & -1.88844100 \\
\hline $\mathrm{C}$ & 9.92038000 & -10.30229100 & -1.63020100 \\
\hline $\mathrm{H}$ & 10.07578800 & -10.40030300 & -0.54938200 \\
\hline $\mathrm{H}$ & 9.45792500 & -11.21983000 & -2.01877100 \\
\hline $\mathrm{H}$ & 10.88478300 & -10.13263200 & -2.12733300 \\
\hline $\mathrm{O}$ & 6.45916400 & 4.23185200 & 0.48545000 \\
\hline $\mathrm{O}$ & 7.85584300 & -0.11723400 & -2.65281000 \\
\hline $\mathrm{C}$ & 9.05985400 & 0.02801600 & -0.11794600 \\
\hline $\mathrm{H}$ & 9.75157700 & 0.48030700 & 0.60449700 \\
\hline $\mathrm{H}$ & 9.65270100 & -0.38832300 & -0.94316400 \\
\hline $\mathrm{C}$ & 8.10145900 & 1.09423600 & -0.63502900 \\
\hline $\mathrm{C}$ & 7.77900300 & 2.22055400 & 0.13053600 \\
\hline $\mathrm{H}$ & 8.31650400 & 2.38839400 & 1.06529300 \\
\hline $\mathrm{C}$ & 6.75841300 & 3.10379500 & -0.24969800 \\
\hline $\mathrm{C}$ & 5.96171000 & 2.82841500 & -1.37300000 \\
\hline $\mathrm{C}$ & 6.32568700 & 1.73532700 & -2.17427700 \\
\hline $\mathrm{H}$ & 5.72890200 & 1.54925300 & -3.06772100 \\
\hline $\mathrm{C}$ & 7.40687400 & 0.91013900 & -1.85009400 \\
\hline $\mathrm{C}$ & 7.51011600 & 5.05363500 & 0.95289600 \\
\hline $\mathrm{H}$ & 7.82383900 & 4.76214200 & 1.97219900 \\
\hline $\mathrm{H}$ & 8.39252700 & 4.98262600 & 0.29332500 \\
\hline
\end{tabular}




\begin{tabular}{|c|c|c|c|}
\hline $\mathrm{C}$ & 7.30454600 & -0.31352200 & -3.93929700 \\
\hline $\mathrm{H}$ & 7.61709700 & -1.32155800 & -4.24861500 \\
\hline $\mathrm{H}$ & 6.20267200 & -0.30525400 & -3.90815300 \\
\hline $\mathrm{C}$ & 4.72801800 & 3.64756200 & -1.70488800 \\
\hline $\mathrm{H}$ & 4.61067200 & 3.70573800 & -2.79644700 \\
\hline $\mathrm{H}$ & 4.87142200 & 4.67435900 & -1.33760600 \\
\hline $\mathrm{O}$ & -8.32346200 & -0.48867200 & -2.31328000 \\
\hline $\mathrm{O}$ & -6.68994800 & 4.42265900 & -0.32745800 \\
\hline $\mathrm{N}$ & 0.17456800 & 1.12328500 & 0.71873000 \\
\hline $\mathrm{N}$ & -1.00903400 & 0.96497000 & 0.39700100 \\
\hline $\mathrm{C}$ & 2.19264600 & 2.38447400 & 0.86187400 \\
\hline $\mathrm{H}$ & 2.11027300 & 2.23701600 & 1.94045100 \\
\hline $\mathrm{C}$ & 3.31941100 & 2.97272000 & 0.30118600 \\
\hline $\mathrm{H}$ & 4.12307900 & 3.33181300 & 0.94750500 \\
\hline $\mathrm{C}$ & 3.45492900 & 3.07487900 & -1.09528000 \\
\hline $\mathrm{C}$ & 2.40412500 & 2.60899600 & -1.90038300 \\
\hline $\mathrm{H}$ & 2.48937300 & 2.67421200 & -2.98831900 \\
\hline $\mathrm{C}$ & 1.24658800 & 2.05175000 & -1.34590200 \\
\hline $\mathrm{H}$ & 0.46450500 & 1.65914900 & -1.99494400 \\
\hline $\mathrm{C}$ & 1.14003700 & 1.92622600 & 0.04801100 \\
\hline $\mathrm{C}$ & -1.78715700 & 1.70142900 & -0.54079800 \\
\hline $\mathrm{C}$ & -2.39982000 & 1.00218500 & -1.59663100 \\
\hline $\mathrm{H}$ & -2.05870400 & -0.00939100 & -1.82841900 \\
\hline $\mathrm{C}$ & -3.43625600 & 1.59794000 & -2.30650200 \\
\hline $\mathrm{H}$ & -3.89875200 & 1.04037800 & -3.12518100 \\
\hline $\mathrm{C}$ & -3.92961800 & 2.86834900 & -1.96314900 \\
\hline $\mathrm{C}$ & -3.28003600 & 3.56991000 & -0.93977000 \\
\hline $\mathrm{H}$ & -3.63638300 & 4.56320400 & -0.65625900 \\
\hline $\mathrm{C}$ & -2.20428600 & 3.00802300 & -0.24374700 \\
\hline $\mathrm{H}$ & -1.73170100 & 3.55392800 & 0.57557000 \\
\hline
\end{tabular}




\begin{tabular}{|c|c|c|c|}
\hline $\mathrm{C}$ & -5.20787900 & 3.39098900 & -2.60550000 \\
\hline $\mathrm{H}$ & -5.27819300 & 4.47707500 & -2.46115900 \\
\hline $\mathrm{H}$ & -5.18954200 & 3.20264300 & -3.68967700 \\
\hline $\mathrm{C}$ & -6.40563900 & 2.69137000 & -1.98688900 \\
\hline $\mathrm{C}$ & -6.85369400 & 1.46184400 & -2.49798500 \\
\hline $\mathrm{H}$ & -6.41220500 & 1.11470700 & -3.43188000 \\
\hline $\mathrm{C}$ & -7.80359500 & 0.68812300 & -1.82303700 \\
\hline $\mathrm{C}$ & -8.27631200 & 1.08758400 & -0.55386400 \\
\hline $\mathrm{C}$ & -7.89886300 & 2.35368900 & -0.09633900 \\
\hline $\mathrm{H}$ & -8.27327300 & 2.71324800 & 0.86361200 \\
\hline $\mathrm{C}$ & -7.01093000 & 3.16397900 & -0.81396300 \\
\hline $\mathrm{C}$ & -9.07061600 & 0.12222300 & 0.31353300 \\
\hline $\mathrm{H}$ & -9.81904200 & -0.40551100 & -0.29266700 \\
\hline $\mathrm{H}$ & -9.60128400 & 0.69149400 & 1.08893100 \\
\hline $\mathrm{C}$ & -7.81414800 & -1.05463900 & -3.50189100 \\
\hline $\mathrm{H}$ & -6.71396300 & -0.99184500 & -3.53331100 \\
\hline $\mathrm{H}$ & -8.08361300 & -2.12079400 & -3.47106400 \\
\hline $\mathrm{C}$ & -7.53352700 & 5.46272900 & -0.78673900 \\
\hline $\mathrm{H}$ & -8.56312900 & 5.33558100 & -0.40169700 \\
\hline $\mathrm{H}$ & -7.59265500 & 5.47036200 & -1.89110700 \\
\hline $\mathrm{C}$ & -8.37504900 & -0.41679800 & -4.77743800 \\
\hline $\mathrm{H}$ & -7.78702300 & -0.75365100 & -5.64213000 \\
\hline $\mathrm{H}$ & -8.29789100 & 0.67805400 & -4.73479400 \\
\hline $\mathrm{C}$ & -6.93429700 & 6.77545700 & -0.27854600 \\
\hline $\mathrm{H}$ & -5.94274400 & 6.93936800 & -0.72151400 \\
\hline $\mathrm{H}$ & -6.80562400 & 6.72755300 & 0.81187100 \\
\hline $\mathrm{C}$ & 7.76569100 & 0.71495700 & -4.97712900 \\
\hline $\mathrm{H}$ & 7.14499600 & 0.61837400 & -5.87870500 \\
\hline $\mathrm{H}$ & 7.63736900 & 1.73705300 & -4.59141800 \\
\hline $\mathrm{C}$ & 6.95165900 & 6.48286400 & 0.95612700 \\
\hline
\end{tabular}




\begin{tabular}{|c|c|c|c|}
\hline $\mathrm{H}$ & 6.70083500 & 6.78719800 & -0.06859000 \\
\hline $\mathrm{H}$ & 6.02461100 & 6.51327200 & 1.54614000 \\
\hline $\mathrm{N}$ & 7.87815600 & 7.47659400 & 1.51027000 \\
\hline $\mathrm{C}$ & 8.56728700 & 8.39065400 & 0.80383800 \\
\hline $\mathrm{C}$ & 8.15643800 & 7.66688800 & 2.85427400 \\
\hline $\mathrm{H}$ & 8.54237300 & 8.50717000 & -0.27790400 \\
\hline $\mathrm{H}$ & 7.69857900 & 7.06480700 & 3.63436600 \\
\hline $\mathrm{N}$ & -9.77489300 & -0.76143500 & -5.05978500 \\
\hline $\mathrm{C}$ & -10.20062900 & -1.68001200 & -6.00495700 \\
\hline $\mathrm{C}$ & -10.85666300 & -0.21579700 & -4.47980700 \\
\hline $\mathrm{C}$ & -11.56759700 & -1.67258700 & -5.98518700 \\
\hline $\mathrm{H}$ & -9.50683100 & -2.24214000 & -6.62522600 \\
\hline $\mathrm{H}$ & -10.83978000 & 0.54453400 & -3.70296500 \\
\hline $\mathrm{H}$ & -12.28939300 & -2.22904400 & -6.57965100 \\
\hline $\mathrm{N}$ & -7.77384700 & 7.93823200 & -0.58960400 \\
\hline $\mathrm{C}$ & -8.92263300 & 8.31025800 & 0.08971700 \\
\hline $\mathrm{C}$ & -7.56003300 & 8.82827400 & -1.57463100 \\
\hline $\mathrm{C}$ & -9.39419700 & 9.44556600 & -0.50838100 \\
\hline $\mathrm{H}$ & -9.29881200 & 7.75485700 & 0.94438600 \\
\hline $\mathrm{H}$ & -6.72729000 & 8.81219700 & -2.27479400 \\
\hline $\mathrm{H}$ & -10.25999100 & 10.06337400 & -0.27907700 \\
\hline $\mathrm{N}$ & 9.16570600 & 0.55759700 & -5.39682900 \\
\hline $\mathrm{C}$ & 9.58727500 & 0.47150500 & -6.67065900 \\
\hline $\mathrm{C}$ & 10.27534700 & 0.55340100 & -4.56770600 \\
\hline $\mathrm{H}$ & 8.94887000 & 0.46558100 & -7.55252200 \\
\hline $\mathrm{C}$ & 11.37565500 & 0.45779000 & -5.37564600 \\
\hline $\mathrm{H}$ & 10.17998800 & 0.61398600 & -3.48835500 \\
\hline $\mathrm{H}$ & 12.43569900 & 0.42474500 & -5.13445100 \\
\hline $\mathrm{N}$ & 10.92220000 & 0.40909500 & -6.68053200 \\
\hline $\mathrm{N}$ & -8.52857400 & 9.74887000 & -1.54249700 \\
\hline
\end{tabular}




\begin{tabular}{|c|c|c|c|}
\hline $\mathrm{N}$ & -11.95332300 & -0.75225900 & -5.02851400 \\
\hline $\mathrm{C}$ & -13.34406100 & -0.40059500 & -4.71214800 \\
\hline $\mathrm{H}$ & -13.82708300 & 0.01873200 & -5.60549400 \\
\hline $\mathrm{H}$ & -13.88578800 & -1.29957500 & -4.38901300 \\
\hline $\mathrm{H}$ & -13.35322200 & 0.34476900 & -3.90831900 \\
\hline $\mathrm{C}$ & -8.65080800 & 10.91156200 & -2.43563400 \\
\hline $\mathrm{H}$ & -9.61837600 & 10.87298800 & -2.95370700 \\
\hline $\mathrm{H}$ & -8.58270200 & 11.83457100 & -1.84388600 \\
\hline $\mathrm{H}$ & -7.83868000 & 10.88671800 & -3.17159800 \\
\hline $\mathrm{C}$ & 11.77476400 & 0.31981700 & -7.87690200 \\
\hline $\mathrm{H}$ & 12.43026100 & 1.19995600 & -7.92395100 \\
\hline $\mathrm{H}$ & 12.38124700 & -0.59433100 & -7.82662000 \\
\hline $\mathrm{H}$ & 11.13941700 & 0.28970300 & -8.76994200 \\
\hline $\mathrm{C}$ & 9.02994400 & 8.71537000 & 2.93941600 \\
\hline $\mathrm{H}$ & 9.48576200 & 9.19307600 & 3.80419300 \\
\hline $\mathrm{N}$ & 9.27038700 & 9.14985200 & 1.64928800 \\
\hline $\mathrm{C}$ & 10.13580900 & 10.28269800 & 1.28341400 \\
\hline $\mathrm{H}$ & 10.13238200 & 10.40093000 & 0.19348700 \\
\hline $\mathrm{H}$ & 9.75253600 & 11.19904200 & 1.75251600 \\
\hline $\mathrm{H}$ & 11.15937700 & 10.08748900 & 1.63026700 \\
\hline
\end{tabular}

